MLM-3802

UC-701

\title{
Iron/Potassium Perchlorate Pellet Burn Rate Measurements
}

\author{
John W. Reed and Ronald R. Walters
}

Issued: January 25, 1995

DISCLAIMER

This report was prepared as an account of work sponsored by an agency of the United States Government. Neither the United States Government nor any agency thereof, nor any of their employees, makes any warranty, express or implied, or assumes any legal liability or responsibility for the accuracy, completeness, or usefulness of any information, apparatus, product, or process disclosed, or represents that its use would not infringe privately owned rights. Reference herein to any specific commercial product, process, or service by trade name, trademark, manufacturer, or otherwise does not necessarily constitute or imply its endorsement, recommendation, or favoring by the United States Government or any agency thereof. The views and opinions of authors expressed herein do not necessarily state or reflect those of the United States Government or any agency thereof.

MOUND

operated by

EGRL MOUND APPLIED TECHNOLOGIES

P.O. Box 3000, Miamisburg, Ohio 45343-3000

for the

U. S. DEPARTMENT OF ENERGY

Contract No. DE-AC04-88DP43495 


\section{Contents}

\section{page}

Abstract $\ldots \ldots \ldots \ldots \ldots \ldots \ldots \ldots \ldots \ldots \ldots \ldots \ldots \ldots \ldots \ldots \ldots \ldots \ldots$

Introduction $\ldots \ldots \ldots \ldots \ldots \ldots \ldots \ldots \ldots \ldots \ldots \ldots \ldots \ldots \ldots \ldots$

Experimental ........................... 4

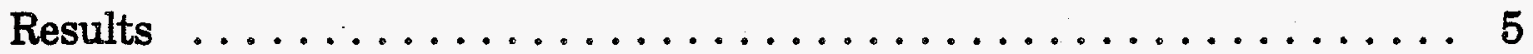

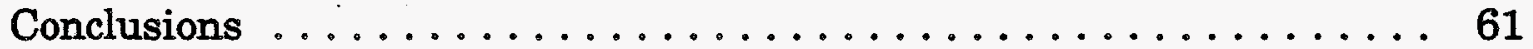

Reference ............................. 66

Distribution .......................... 67 


\section{DISCLAIMER}

Portions of this document may be illegible in electronic image products. Images are produced from the best available original document. 


\section{Abstract}

A burn rate test having several advantages for low gas-producing pyrotechnic compacts has been developed. The technique involves use of a high speed video motion analysis system that allows immediate turnaround and produces all required data for rate computation on magnetic tape and becomes immediately available on the display screen. The test technique provides a quick method for material qualification along with data for improved reliability and function.

Burn rate data has been obtained for both UPI and Eagle Pitcher Iron / Potassium Perchlorate blends. The data obtained for the UPI blends cover a range of composition, pellet density, and ambient (before ignition) pellet temperature. Burn rate data for the E-P blends were extended to include surface conditions or particle size as a variable parameter.

\section{Introduction}

Burn rate tests are often used as material qualification tests and as means of predicting performance of pyrotechnic blends. For example, in development of propellant or heat source blends, the rate of energy production is extremely dependent on burn rate, among other parameters. The driving interest in this work was to improve the function and performance of the Iron/Potassium Perchlorate pyrotechnic blend used as a heat source to drive thermal batteries.

The objective of the work described here is to develop a rapid method for measuring the burn rate of gasless pyrotechnic heat sources and to then 
measure and relate burn rate data to various parameters known and suspected to contribute to burn rate variations in the system iron/potassium perchlorate. Several parameters are deliberately varied in device applications to tailor the burn rate to satisfy device requirements. These parameters have usually been the relative composition of tine blend components and the density of the compact. Variations in physical properties of the materials composing the blend also contribute to variation in burn rate of the blend compacts.

\section{Expérimental}

The burn rate apparatus consists of a controlled temperature surface on which the pyrotechnic sample pellet rests. Various substrate materials may be inserted between that surface and the sample pellet. In this manner, the physical properties of the substrate material can be varied, and the effects of this variation on the burn rate may be observed.

The temperature of the surface can be varied from less than $-54^{\circ} \mathrm{C}$ to temperatures in excess of $150^{\circ} \mathrm{C}$. It has been found that the temperature of the sample pellet varies less than $3^{\circ} \mathrm{C}$ from the measured temperature of the underlying surface during an observation. Because the control temperature is not usually very far from ambient in this work, a fair amount of temperature oscillation can be observed.

Ignition is initiated when the temperature of the pellet reaches the required temperature. An optical quartz cover is used to prevent separation of the pellet from the substrate during burning and to provide a consistent surface contact with the upper face of the pellet. The cover is three inches in diameter and one quarter inch thick, covering two or three pellets. Initiation is by electric match. The intent is to measure duplicate or triplicate samples by propagation of the burn from one pellet to another across an edge.

A high speed video motion analysis system is used to observe the motion of the burn front across the surface of the pellet and to measure the time and distance associated with this process. The high temperature reaction front can be easily followed and recorded on video tape. The record rate can be varied from standard VHS rates to 12,000 events per second. For this work, 
recording at 1000 frames per second has been sufficient to capture these events. Each frame recorded and stored on tape contains the position of the reaction front and an associated elapsed time from an arbitrary time base. The motion analysis system also provides a means of measurement of the position of the burn front using video screen reticles. This allows the measurement of distance intervals across a convenient number of frames sufficient to calculate reliable burn rate values. The high speed video tape provides a permanent record of the events.

\section{Results}

The first set of burn rate measurements was done with the object of determining the effect on burn rate of the substrate in contact with the $\mathrm{Fe} / \mathrm{KClO}_{4}$ (88/12) pellet. All burn rate determinations were made on 1.5-inch diameter pellets. The substrate material was chosen so that a wide range of specific heat and thermal conductivity would be represented. The materials were copper, aluminum, graphite, quartz, and an insulating material, MinK. Quartz was included to determine its effect as a covering material, which was used throughout the complete series of measurements after it was discovered that a cover was required to obtain reproducible results. This was probably the effect of the reduction of nonreproducible heat loss caused by the hot, volatile potassium chloride that was produced in the pyrotechnic reaction. With the quartz cover plate in place, the sample pellet did not move or deform as it did with no cover, thus allowing much of the potassium chloride to remain condensed between the pellet and the substrate and cover.

The effect of the substrate material on the burn rate of $\mathrm{Fe} / \mathrm{KClO}_{4}$ pellets is small, as can be seen in Figure 1, where the variation in rate between substrates is shown to be about the same as the spread observed for each individual substrate.

Pellet thickness has no effect on burn rate over the thickness range observed, as can be seen from the data of Figure 2, where the results of 


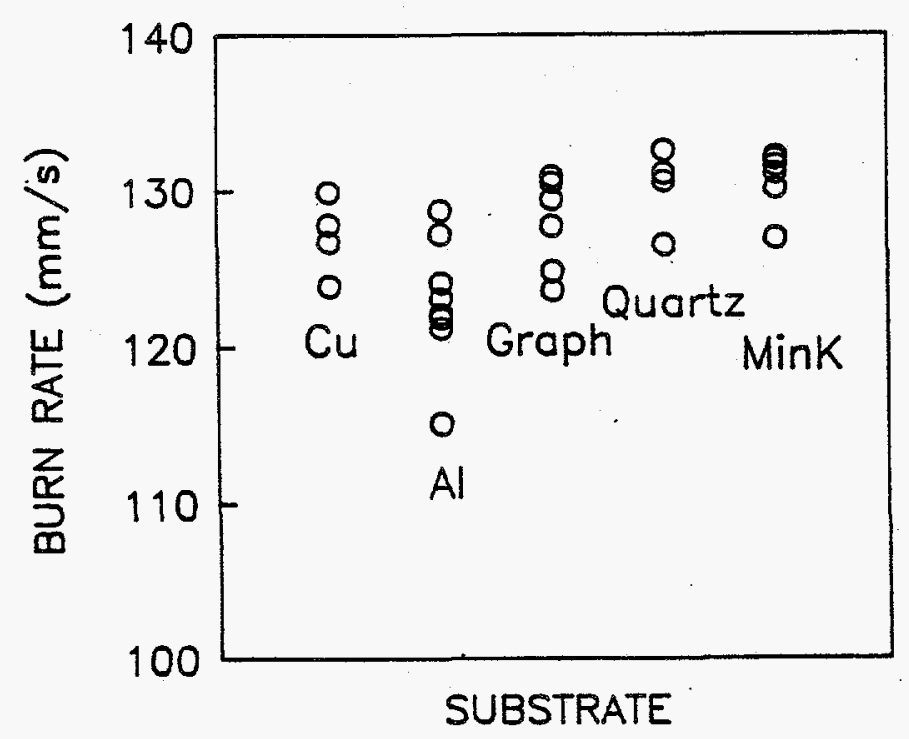

Figure 1 - Burn rates of $\mathrm{Fe} / \mathrm{K}(88 / 12)$, density 3.35 show small variation with adjacent substrate.

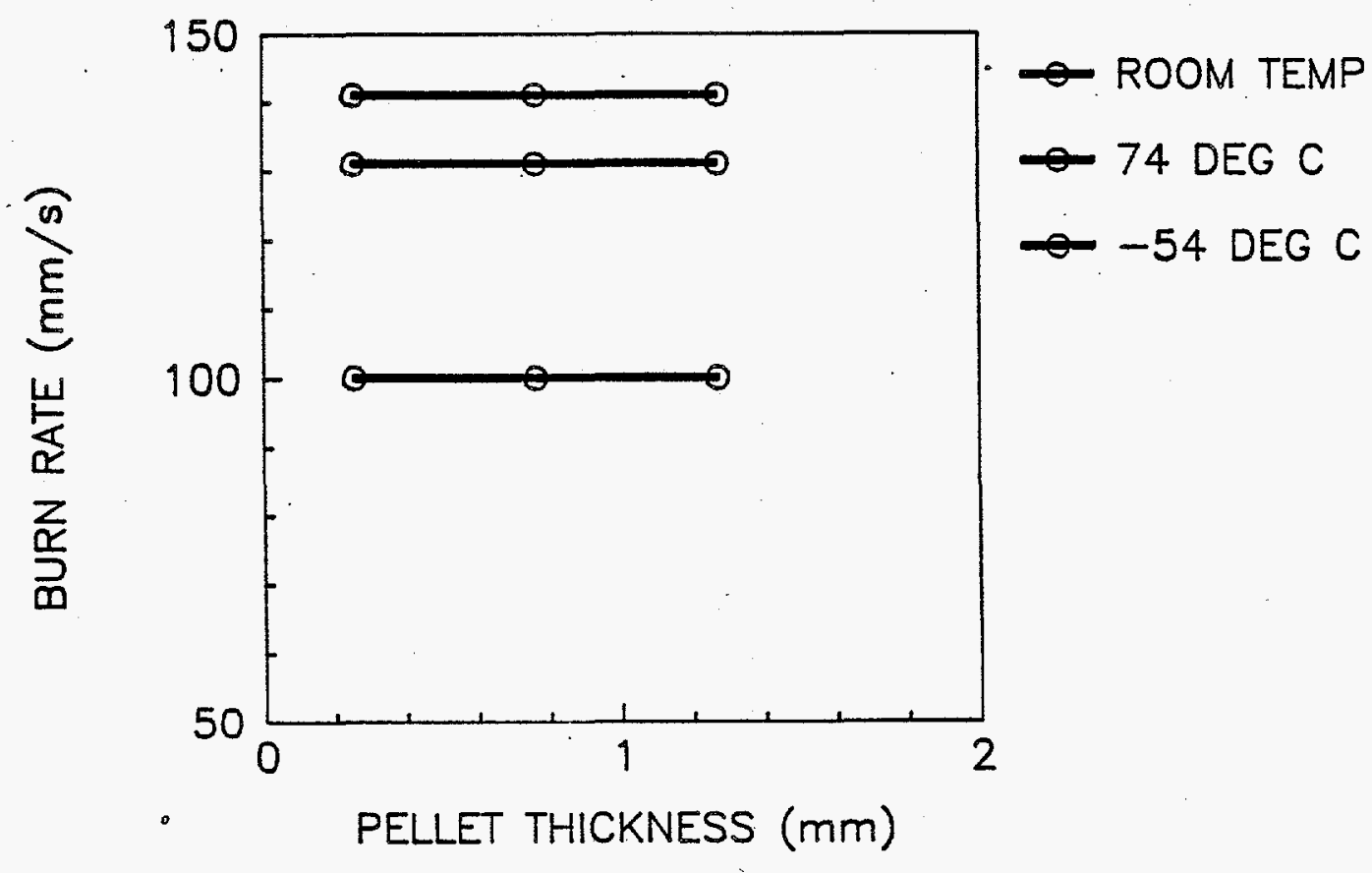

Figure 2 - Burn rate of $\mathrm{Fe} / \mathrm{K}(88 / 12)$ pellets shows no variation with thickness. 
determinations on pellets at three temperatures and three thicknesses are shown. The rather pronounced effect of temperature on burn rate is, however, shown very nicely here. At some small value of thickness it would be expected that the effect of thermal conductivity would be great enough to remove heat from the bulk of the pellet and decrease the propagation rate, but the effect cannot yet be seen within this thickness range. Of the variables investigated in this series of determinations (temperature, substrate properties, and pellet thickness) only temperature was observed to have had a significant effect.

A second set of burn rate measurements was done on samples of $\mathrm{Fe} / \mathrm{KClO}_{4}$ blended at UPI for comparison purposes and to determine what effect the fuel-to-oxidizer ratio $\left(\mathrm{Fe} / \mathrm{KClO}_{4}\right)$, pellet density, and pre-ignition pellet temperature had on the burn rate. The burn rate measurements were done over a range of densities from 3.0 to $4.0 \mathrm{~g} / \mathrm{mL}$. The composition range covered $\mathrm{Fe} / \mathrm{KClO}_{4}$ ratios $84 / 16,86 / 14$, and $88 / 12$, and the temperatures covered were $-54^{\circ} \mathrm{C}$ and $74^{\circ} \mathrm{C}$.

Figures 3 through 9 show the burn rate data obtained from this series of determinations. The combination of parameters chosen for each plot was chosen to show the variation in burn rate with the variables in an easily understandable set. Each figure contains several related sets of raw data shown as points and three constant polynomial least square fits for each set, shown as line plots.

Figures 3,4 and 5 show the variation of the burn rate with the independent variable composition $\left(\mathrm{Fe} / \mathrm{KClO}_{4}\right)$ at two values of temperature. Least square fits of burn rate over composition are shown for each temperature. Figures 3,4 , and 5 contain the data obtained for pellet densities $3.0,3.5$ and $4.0 \mathrm{~g} / \mathrm{cc}$ respectively. Figures 6 and 7 show the variation of burn rate with com. position at several values of density along with the line plots showing the least square fits of burn rate over composition for each density value. Figures 6 and 7 contain the data obtained for temperatures $-54^{\circ} \mathrm{C}$ and $74^{\circ}$ C respectively. Again, as in Figures 3 through 7, the burn rate data are shown as plots against density for the several values of composition. Again the least square fits are shown as line plots. Figures 8 and 9 contain the data obtained for temperatures $-54^{\circ} \mathrm{C}$ and $74^{\circ} \mathrm{C}$ respectively. It should be 


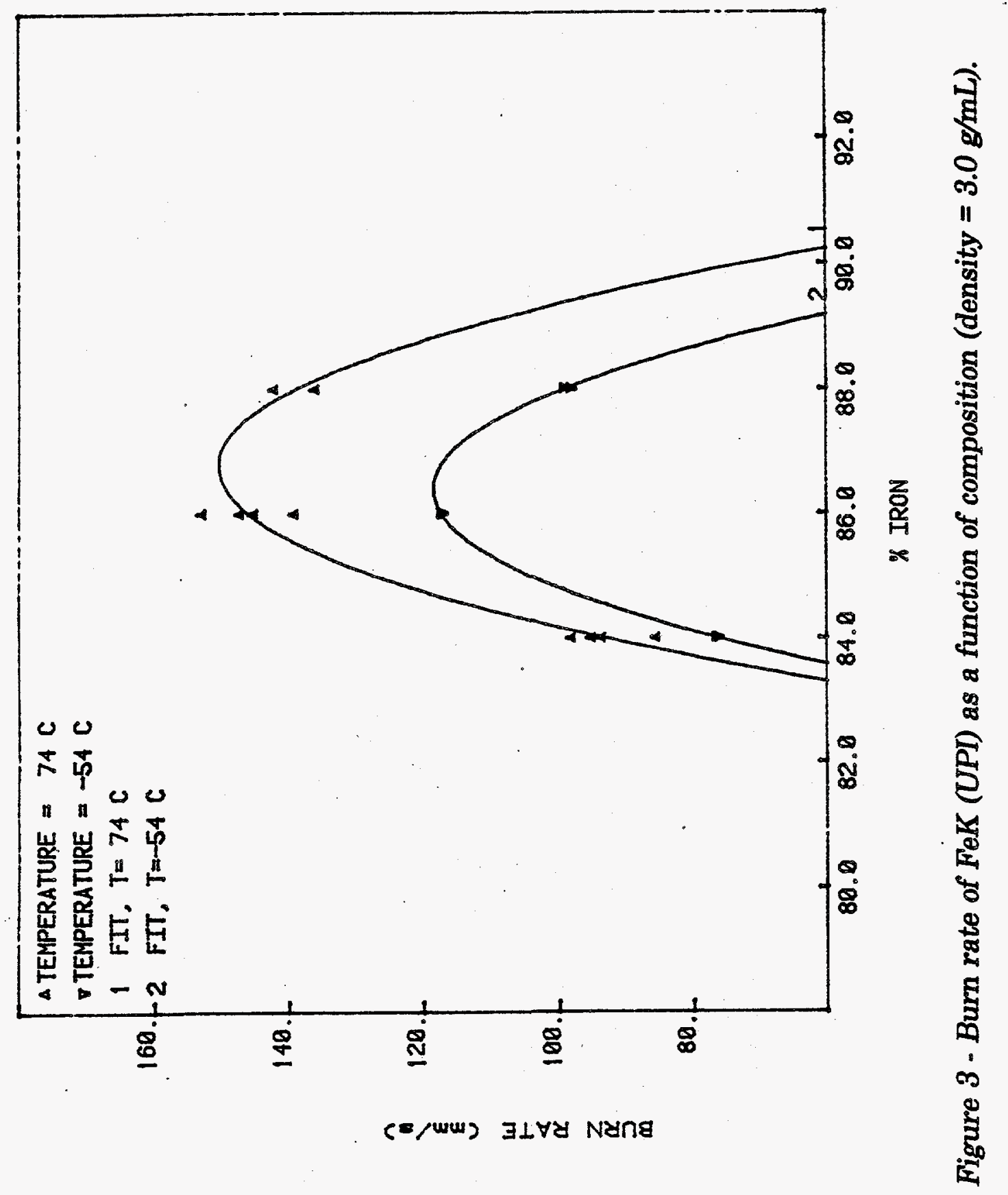




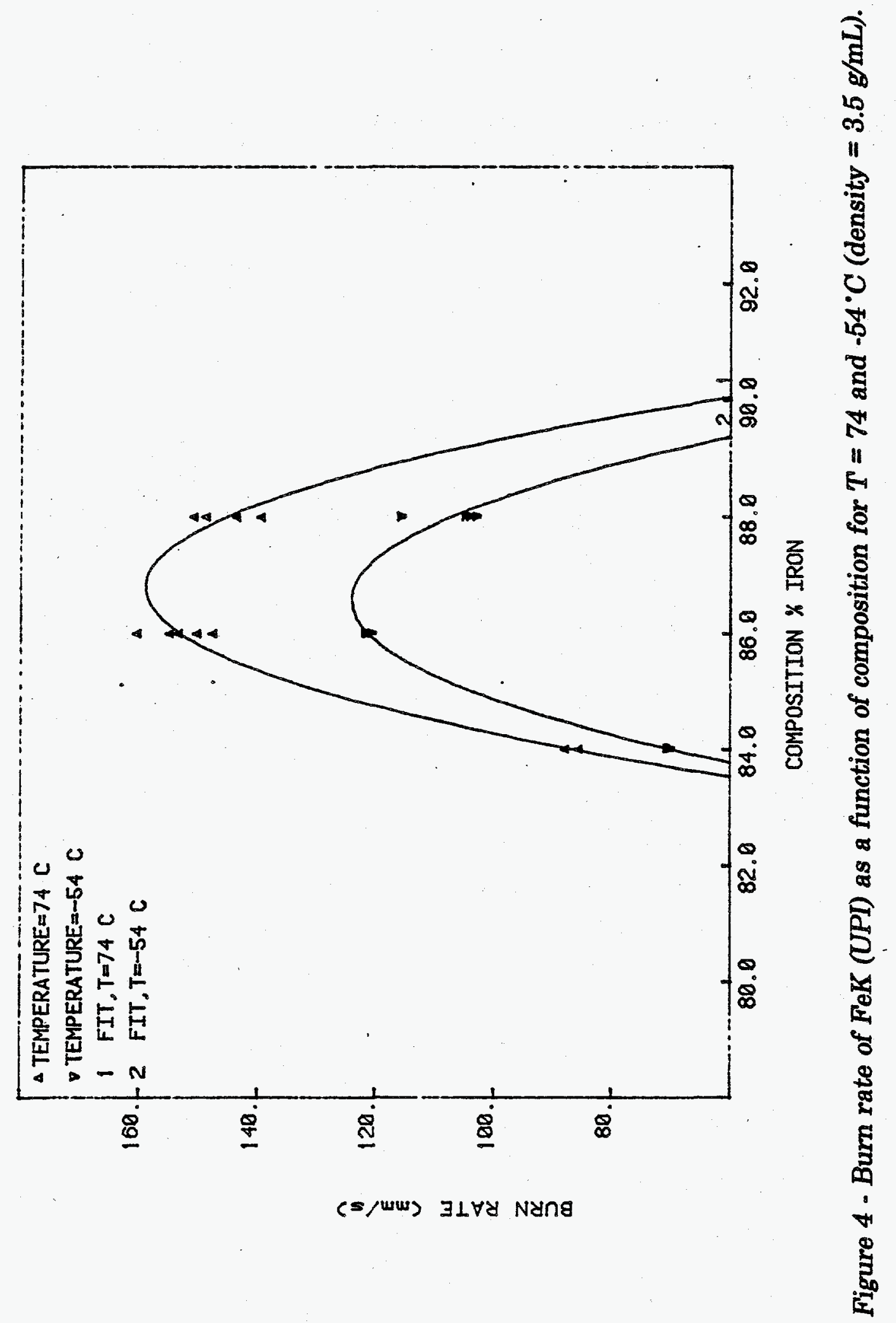




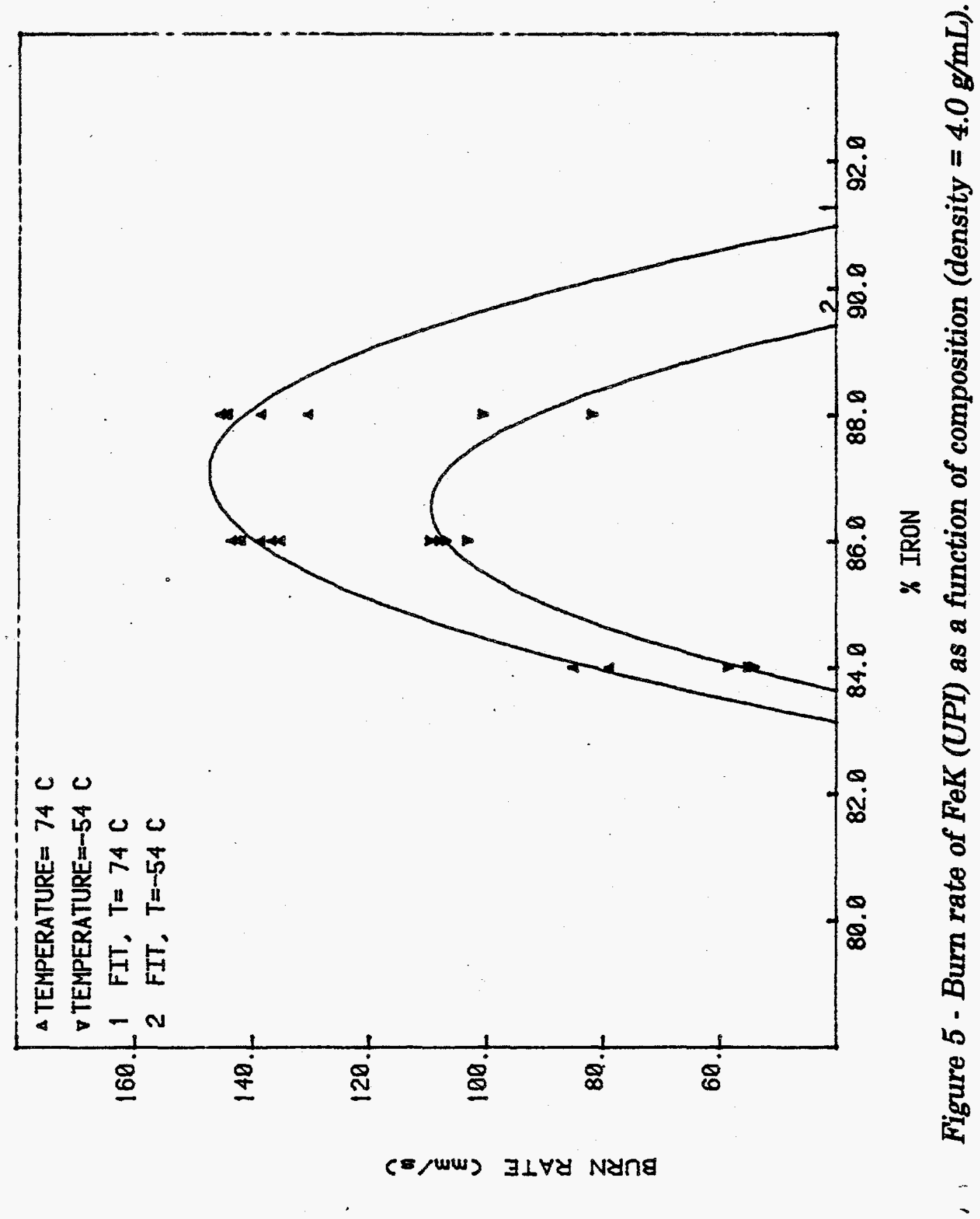




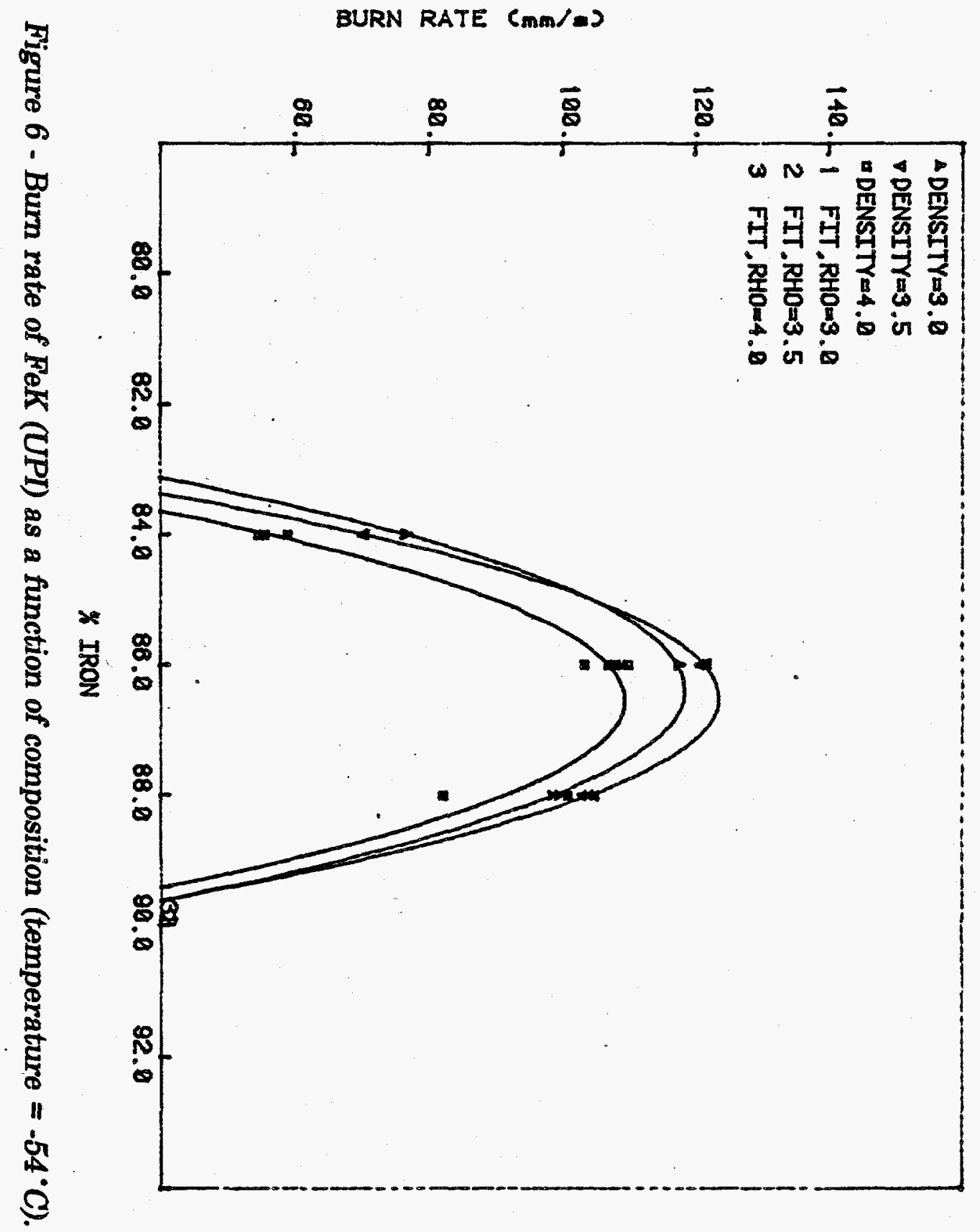




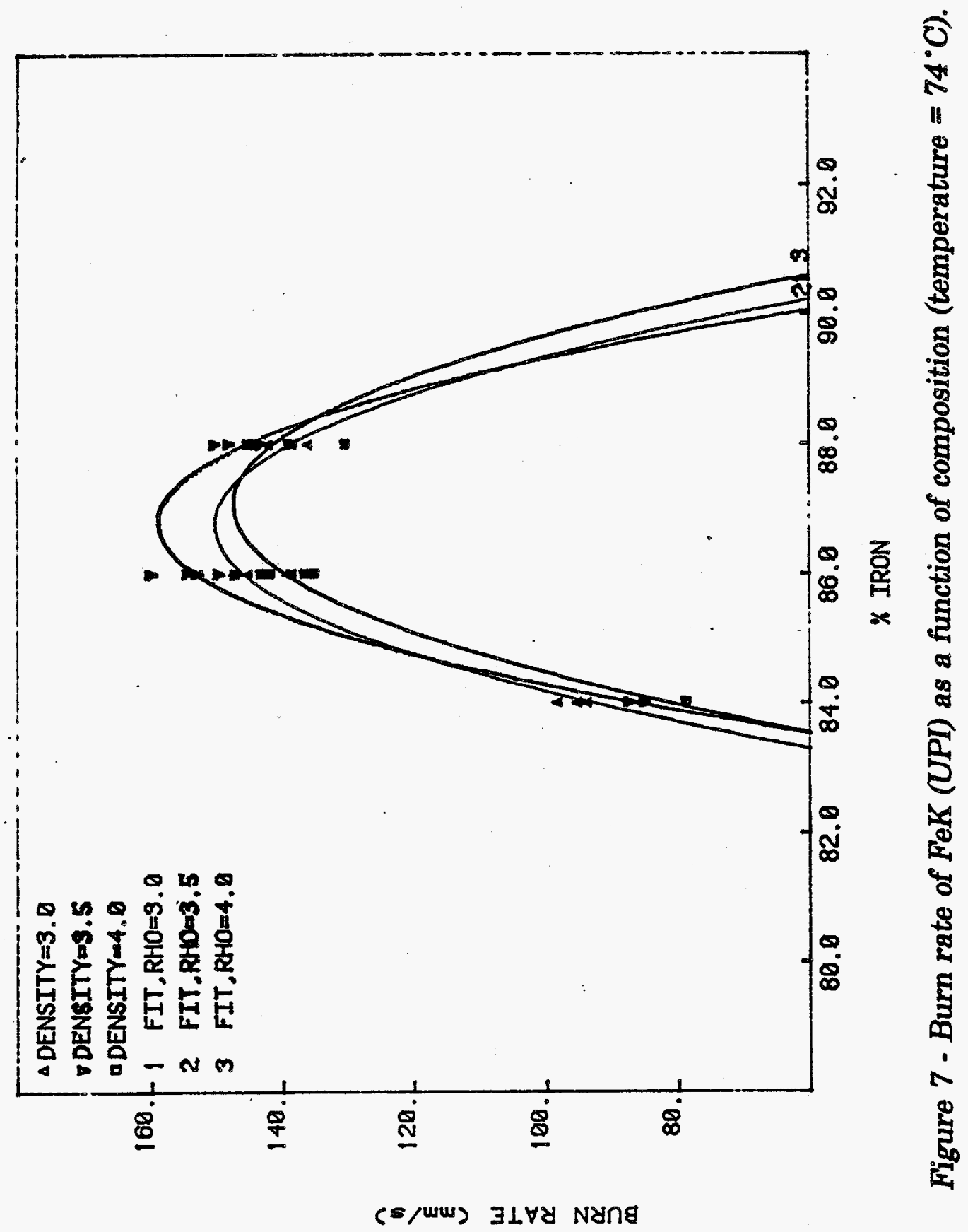




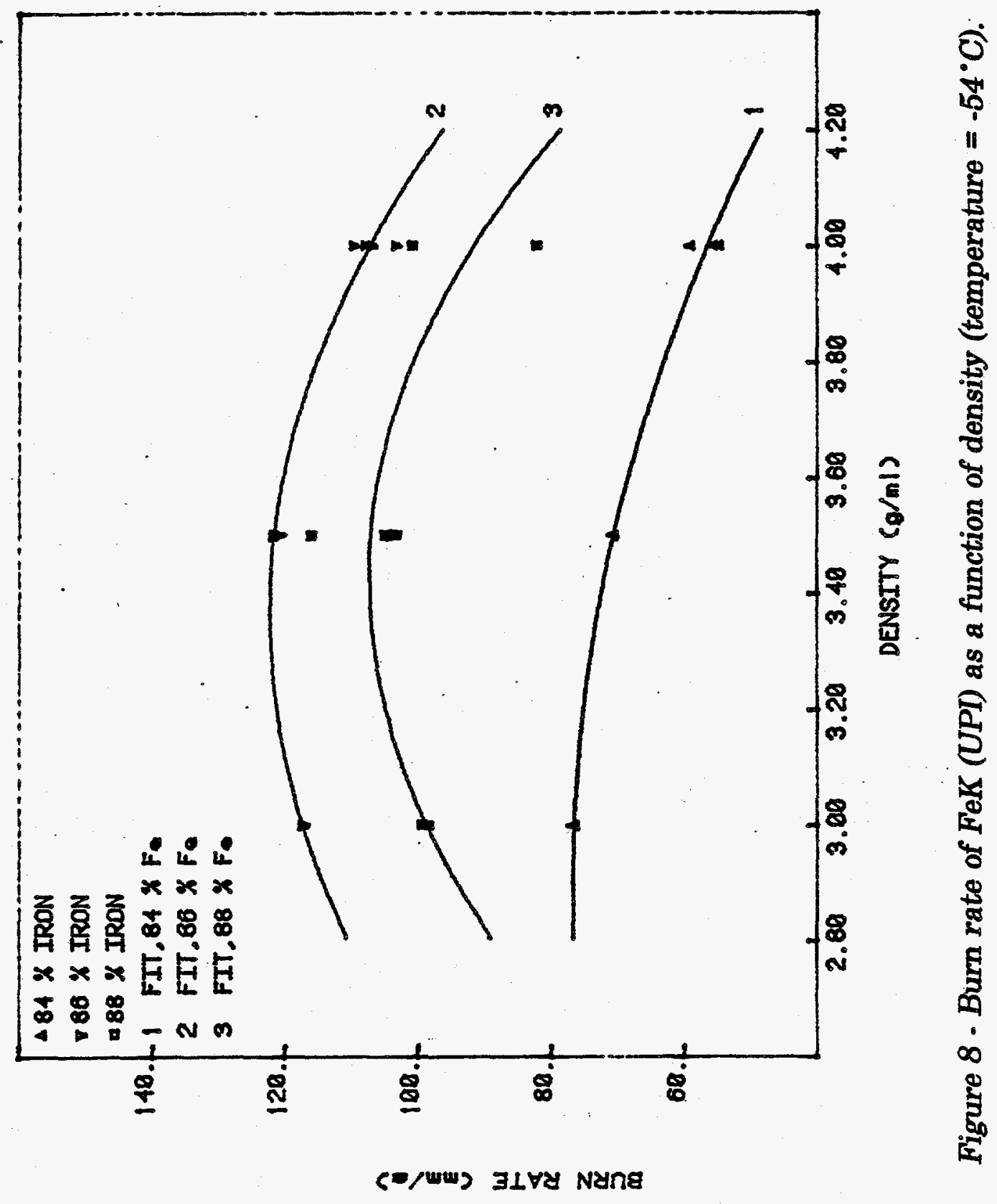




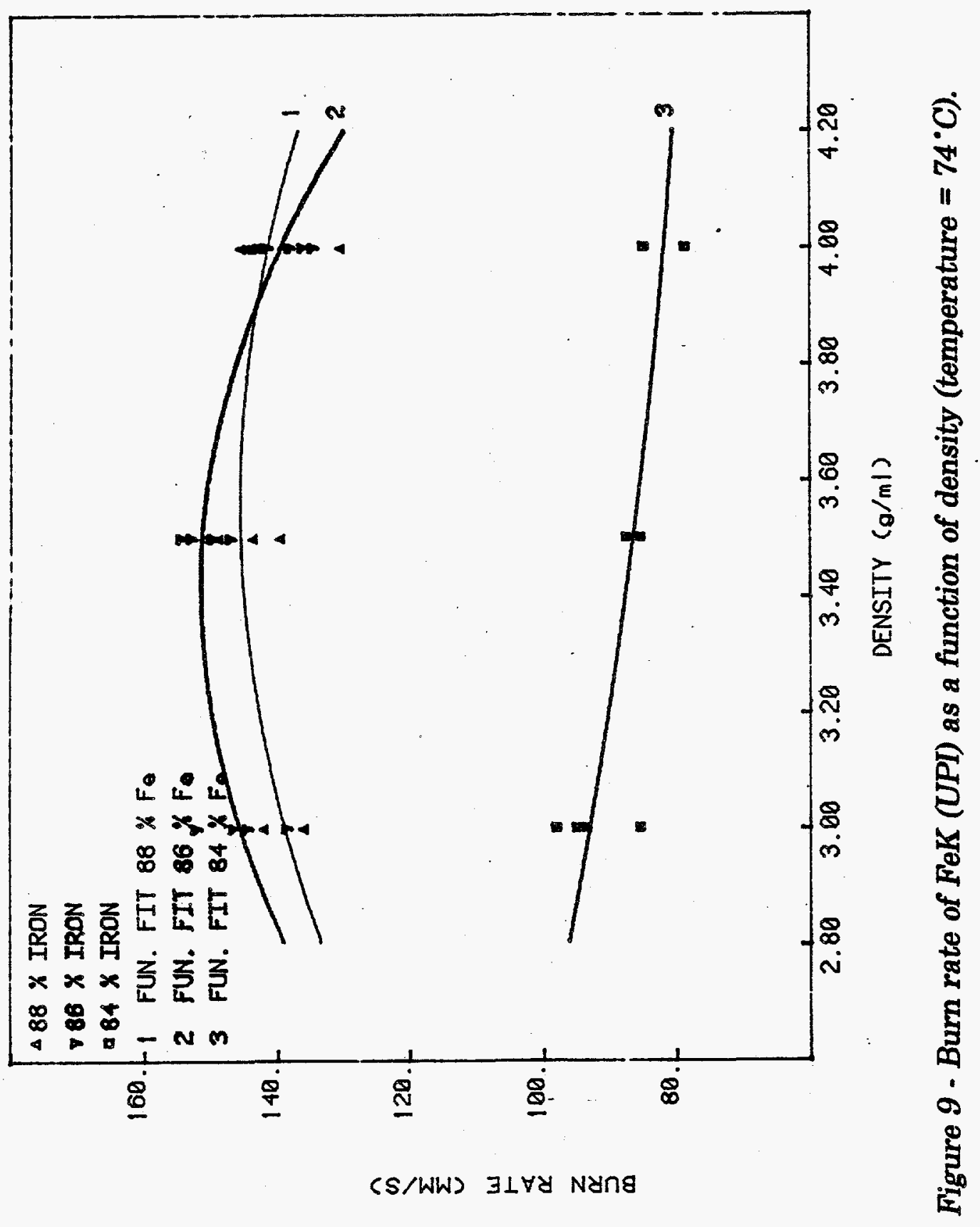


understood that while there are redundant plots of the data, each line plot throughout this series is represented by separate sets of polynomial constants.

To obtain a useful representation of the burn rate data for the UPI blends, all the data was fitted to a seven parameter equation, second order in density, composition, and temperature. The form of the equation for burn rate, $R$, is

$$
\begin{gathered}
\mathrm{R}=\mathrm{A}(1)+\mathrm{A}(2)(\% \mathrm{Fe})+\mathrm{A}(3)(\% \mathrm{Fe})^{2}+\mathrm{A}(4)(\mathrm{g} / \mathrm{mL})+ \\
\mathrm{A}(5)(\mathrm{g} / \mathrm{mL})^{2}+\mathrm{A}(6)(\mathrm{T})+\mathrm{A}(7)(\mathrm{T})^{2}
\end{gathered}
$$

and the parametric constants for the fit are

$$
\begin{aligned}
& A(1)=-57285.7758 \\
& A(2)=178.273214 \\
& A(3)=-26.3931066 \\
& A(4)=0.168684709 \\
& A(5)=0.00436132428 \\
& A(6)=1316.53344 \\
& A(7)=-7.58815575
\end{aligned}
$$

The standard deviation for the fit is $7.1 \mathrm{~mm} / \mathrm{s}$.

The complete UPI burn rate data are contained in Table 1 along with the computed burn rates and the associated deviations. Figures 10 through 16 show the UPI data along with a representation of the fit obtained over all of the parameters for that burn rate data. The data are shown in the same order as in the previous figures, and the raw burn rate data duplicate the previous figures. The line plots, however, represent the above seven parameters fit over the complete set of UPI burn rate data, rather than individual fits for each data set. In general, the plots of the fitting equation represent the data reasonably well over the complete range of data. The line plots representing burn rate as a function of density for $-54^{\circ} \mathrm{C}$ in Figure 15 show the largest deviation and a further inspection of the plotted data may be desirable when using the fitting equation for burn rate estimation in this region. Somewhat tighter fits can be obtained by extending and 


\section{Table 1 - BURN RATE OF FeK (UPI) AS A FUNCTION OF DENSITY, TEMPERATURE AND COMPOSITION}

POLYNOMIAL COEFFICIENTS

B( 1$)=-5.72857758 E+04$
B( 2$)=1.78273214 E+02$
B( 3$)=-2.63931066 E+01$
B( 4$)=1.68684709 E-01$
B( 5$)=4.36132428 E-03$
B( 6$)=1.31653344 E+03$
B( 7$)=-7.58815575 E+00$

\begin{abstract}
DENSITY $\mathrm{g} / \mathrm{ml}$
\end{abstract}
TEMPERATURE C

X IRON

8. $80000000 E+01$

8.80000000E+01

8.80000000E+01

8. $80000000 E+01$

8.80000000E+01

8.80000000E+01

$8.80000000 \mathrm{E}+01$

$8.80000000 E+01$

$8.800000005+01$

$8.80000000 E+01$

8. $80000000 E+01$

8. $80000000 E+01$

8. $80000000 E+01$

8. $80000000 E+01$

$8.60000000 \mathrm{E}+01$

$8.60000000 E+01$

$8.60000000 E+01$

$8.60000000 E+01$

8.60000000E+01

$8.60000000 E+01$

$8.60000000 E+01$

8.60000000E+01

$8.60000000 E+01$

$8.60000000 E+01$

$8.60000000 E+01$

8. $60000000 E+01$

$8.60000000 E+01$

8. $60000000 \mathrm{E}+01$
BURN RATE mm/s

$1.39200000 \mathrm{E}+02$

$1.50600000 E+02$

$1.44000000 E+02$

$1.45200000 \mathrm{E}+02$

$1.43400000 E+02$

$1.48500000 \mathrm{E}+02$

$1.35800000 \mathrm{E}+02$

$1.419000005+02$

$1.44400000 E+02$

$1.45100000 E+02$

1. $40000000 E+02$

$1.55600000 \mathrm{E}+02$

1.30300000E +02

$1.38300000 E+02$

$1.50000000 \mathrm{E}+02$

$1.53200000 \mathrm{E}+02$

$1.34900000 E+02$

$1.43200000 \mathrm{E}+02$

- $1.47000000 \mathrm{E}+02$

$1.52600000 E+02$

$1.60200000 E+02$

$1.47400000 E+02$

$1.54700000 E+02$

$1.39000000 E+02$

$1.45000000 E+02$

$1.38400000 E+02$

$1.42300000 E+02$

$1.36400000 E+02$

CALC

DELTA

$1.43494472 E+02$

$1.43494472 E+02$

$1.33656930 \mathrm{E}+02$

$1.33656930 \mathrm{E}+02$

$1.43494472 E+02$

$1.43494472 E+02$

$1.40135462 E+02$

$1.40135462 E+02$

$1.33656930 E+02$

$1.33656930 E+02$

$1.47800000 E+02$

$1.47800000 \mathrm{E}+02$

$1.33656930 \mathrm{E}+02$

$1.33656930 \mathrm{E}+02$

$1.51105799 \mathrm{E}+02$

$1.51105799 \mathrm{E}+02$

$1.41268257 \mathrm{E}+02$

$1.41268257 \mathrm{E}+02$

$1.47746789 E+02$

$1.47746789 E+02$

$1.51105799 E+02$

$1.51105799 E+02$

$1.51105799 E+02$

$1.47746789 \mathrm{E}+02$

$1.47746789 E+02$

$1.41268257 \mathrm{E}+02$

$1.41268257 \mathrm{E}+02$

$1.41268257 \mathrm{E}+02$
$-4.29447234 E+00$

$7.10552766 E+00$

$1.03430702 E+01$

$1.15430702 E+01$

$-9.44723358 E-02$

$5.00552766 \mathrm{E}+00$

$-4.33546162 E+00$

$1.76453838 E+00$

$1.07430702 E+01$

$1.14430702 E+01$

$-7.80000000 E+00$

$7.80000000 \mathrm{E}+00$

$-3.35692976 E+00$

$4.64307024 E+00$

$-1.10579942 E+00$

$2.09420058 \mathrm{E}+00$

$-6.36825684 E+00$

$1.93174316 E+00$

$-7.46788699 \varepsilon-01$

$4.85321130 E+00$

$9.09420058 E+00$

$-3.70579942 E+00$

$3.59420058 E+00$

$-8.74678870 E+00$

$-2.74678870 E+00$

$-2.86825684 E+00$

$1.03174316 E+00$

$-4.86825684 E+00$ 


\section{Table 1 - BURN RATE OF FeK (UPI) AS A FUNCTION OF DENSITY, TEMPERATURE AND COMPOSITTON (continued)}

\begin{tabular}{|c|c|c|c|c|c|c|}
\hline 29 & $4.00000000 E+00$ & $7.40000000 E+01$ & $8.60000000 E+01$ & $1.41700000 E+02$ & $1.41268257 E+02$ & $4.31743162 E-01$ \\
\hline 30 & $3.00000000 E+00$ & $7.40000000 E+01$ & $8.40000000 E+01$ & $9.35000000 \varepsilon+01$ & $9.46528698 E+01$ & $-1.15286978 E+00$ \\
\hline 31 & $3.00000000 E+00$ & $7.40000000 E+01$ & $8.40000000 E+01$ & $8.54000000 E+01$ & $9.46528698 E+01$ & $-9.25286978 E+00$ \\
\hline 32 & $3.00000000 E+00$ & $7.40000000 E+01$ & $8.40000000 E+01$ & $9.50000000 E+01$ & $9.46528698 E+01$ & $3.47130218 E-01$ \\
\hline 33 & $3.00000000 E+00$ & $7.40000000 E+01$ & $8.40000000 E+01$ & $9.80000000 E+01$ & $9.46528698 E+01$ & $3.34713022 E+00$ \\
\hline 34 & $3.50000000 E+00$ & $7.40000000 E+01$ & $8.40000000 E+01$ & $8.54000000 E+01$ & $9.80118805 E+01$ & $-1.26118805 E+01$ \\
\hline 35 & $3.50000000 E+00$ & $7.40000000 E+01$ & $8.40000000 E+01$ & $8.77000000 E+01$ & $9.80118805 E+01$ & $-1.03118805 E+01$ \\
\hline 36 & $4.00000000 E+00$ & $7.40000000 E+01$ & $8.40000000 E+01$ & $7.87000000 \varepsilon+01$ & 8.81743379E+01 & $-9.47433792 E+00$ \\
\hline 37 & $4.00000000 E+00$ & $7.40000000 E+01$ & $8.40000000 E+01$ & $8.49000000 E+01$ & $8.81743379 E+01$ & $-3.27433792 \varepsilon+00$ \\
\hline 38 & $3.50000000 E+00$ & $-5.40000000 E+01$ & $8.80000000 E+01$ & $1.16000000 \mathrm{E}+02$ & $1.10737839 E+02$ & $5.26216056 E+00$ \\
\hline 39 & $4.00000000 E+00$ & $-5.40000000 E+01$ & $8.80000000 E+01$ & $8.22000000 E+01$ & $1.00900297 E+02$ & $-1.87002969 E+01$ \\
\hline 40 & $3.00000000 E+00$ & $-5.40000000 E+01$ & $8.80000000 E+01$ & $9.83000000 E+01$ & $1.07378829 E+02$ & $-9.07882872 E+00$ \\
\hline 41 & $3.00000000 E+00$ & $-5.40000000 E+01$ & $8.80000000 E+01$ & $9.93000000 E+01$ & $1.07378829 E+02$ & $-8.07882872 E+00$ \\
\hline 42 & $4.00000000 E+00$ & $-5.40000000 E+01$ & $8.80000000 E+01$ & $1.00800000 E+02$ & $1.00900297 E+02$ & $-1.00296863 E-01$ \\
\hline 43 & $3.50000000 E+00$ & $-5.40000000 E+01$ & $8.80000000 E+01$ & $1.05000000 E+02$ & $1.10737839 E+02$ & $-5.73783944 E+00$ \\
\hline 44 & $3.50000000 E+00$ & $-5.40000000 E+01$ & $8.80000000 E+01$ & $1.04200000 E+02$ & $1.10737839 E+02$ & $-6.53783944 E+00$ \\
\hline 45 & $3.50000000 E+00$ & $-5.40000000 E+01$ & $8.80000000 E+01$ & $1.03200000 E+02$ & $1.10737839 E+02$ & $-7.53783944 E+00$ \\
\hline 46. & $3.00000000 E+00$ & $-5.40000000 E+01$ & $8.60000000 E+01$ & $1.17200000 \mathrm{E}+02$ & $1.14990156 E+02$ & $2.20984419 E+00$ \\
\hline 47 & $3.00000000 E+00$ & $-5.40000000 E+01$ & $8.60000000 E+01$ & $1.17400000 E+02$ & $1.14990156 E+02$ & 2.40984419E+00 \\
\hline 48 & $3.50000000 E+00$ & $-5.40000000 E+01$ & $8.60000000 E+01$ & $1.21700000 E+02$ & $1.18349167 E+02$ & $3.35083347 E+00$ \\
\hline 49 & $3.50000000 E+00$ & $-5.40000000 E+01$ & $8.60000000 E+01$ & $1.21700000 E+02$ & $1.18349167 E+02$ & $3.35083347 E+00$ \\
\hline 50 & $3.50000000 E+00$ & $-5.40000000 E+01$ & $8.60000000 E+01$ & $1.20900000 E+02$ & $1.18349167 \mathrm{E}+02$ & $2.55083347 E+00$ \\
\hline 59 & $4.00000000 E+00$ & $-5.40000000 E+01$ & $8.60000000 E+01$ & $1.08100000 E+02$ & $1.08511624 E+02$ & $-4.11623945 E-01$ \\
\hline 52 & $4.00000000 E+00$ & $-5.40000000 E+01$ & $8.60000000 E+01$ & $1.07000000 E+02$ & $1.08511624 E+02$ & $-1.51162395 E+00$ \\
\hline 53 & $4.00000000 E+00$ & $-5.40000000 E+01$ & $8.60000000 E+01$ & $1.03400000 E+02$ & $1.08511624 E+02$ & $-5.11162395 E+00$ \\
\hline 54 & $4.00000000 E+00$ & $-5.40000000 E+01$ & $8.60000000 E+01$ & $1.09800000 E+02$ & $1.08511624 E+02$ & $1.28837605 E+00$ \\
\hline 55 & $3.00000000 E+00$ & $-5.40000000 E+01$ & $8.40000000 E+01$ & $7.67000000 E+01$ & $6.18962369 E+01$ & $1.48037631 E+01$ \\
\hline 56 & $3.00000000 E+00$ & $-5.40000000 E+01$ & $8.40000000 E+01$ & $7.63000000 E+01$ & $6.18962369 E+01$ & $1.44037631 E+01$ \\
\hline 57 & 4.00000000E+00 & $-5.40000000 E+01$ & $8.40000000 E+01$ & $5.46000000 E+01$ & $5.54177050 E+01$ & $-8.17705029 E-01$ \\
\hline 58 & $3.50000000 E+00$ & $-5.40000000 E+01$ & $8.40000000 E+01$ & $7.03700000 E+01$ & $6.52552476 \mathrm{E}+01$ & $5.11475239 E+00$ \\
\hline 59 & $3.50000000 E+00$ & $-5.40000000 E+01$ & $8.40000000 E+01$ & $7.06700000 E+01$ & $6.52552476 E+01$ & $5.41475239 E+00$ \\
\hline 60 & $4.00000000 E+00$ & $-5.40000000 E+01$ & $8.40000000 E+01$ & $5.54000000 E+01$ & $5.54177050 E+01$ & $-1.77050288 E-02$ \\
\hline 61 & $4.00000000 E+00$ & $-5.40000000 E+01$ & $8.40000000 E+01$ & $5.89000000 E+01$ & $5.54177050 E+01$ & $3.48229497 E+00$ \\
\hline SIGMA $=$ & 7.12858 & 3963315 & & & & \\
\hline
\end{tabular}




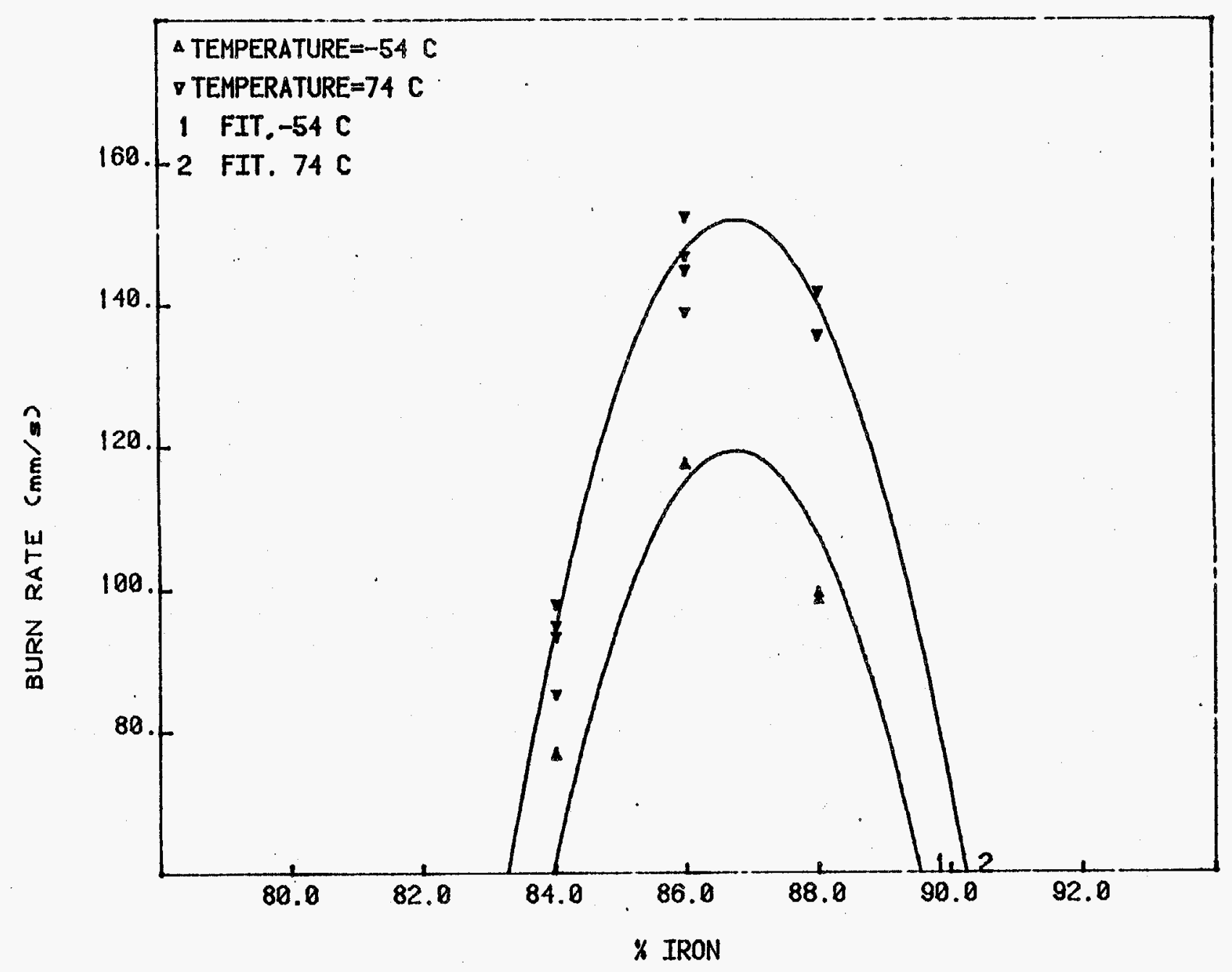

Figure 10 - Burn rate of $\mathrm{FeK}$ (UPI) as a function of composition (density $=3.0 \mathrm{~g} / \mathrm{mL}, 7$ PAR.FIT). 


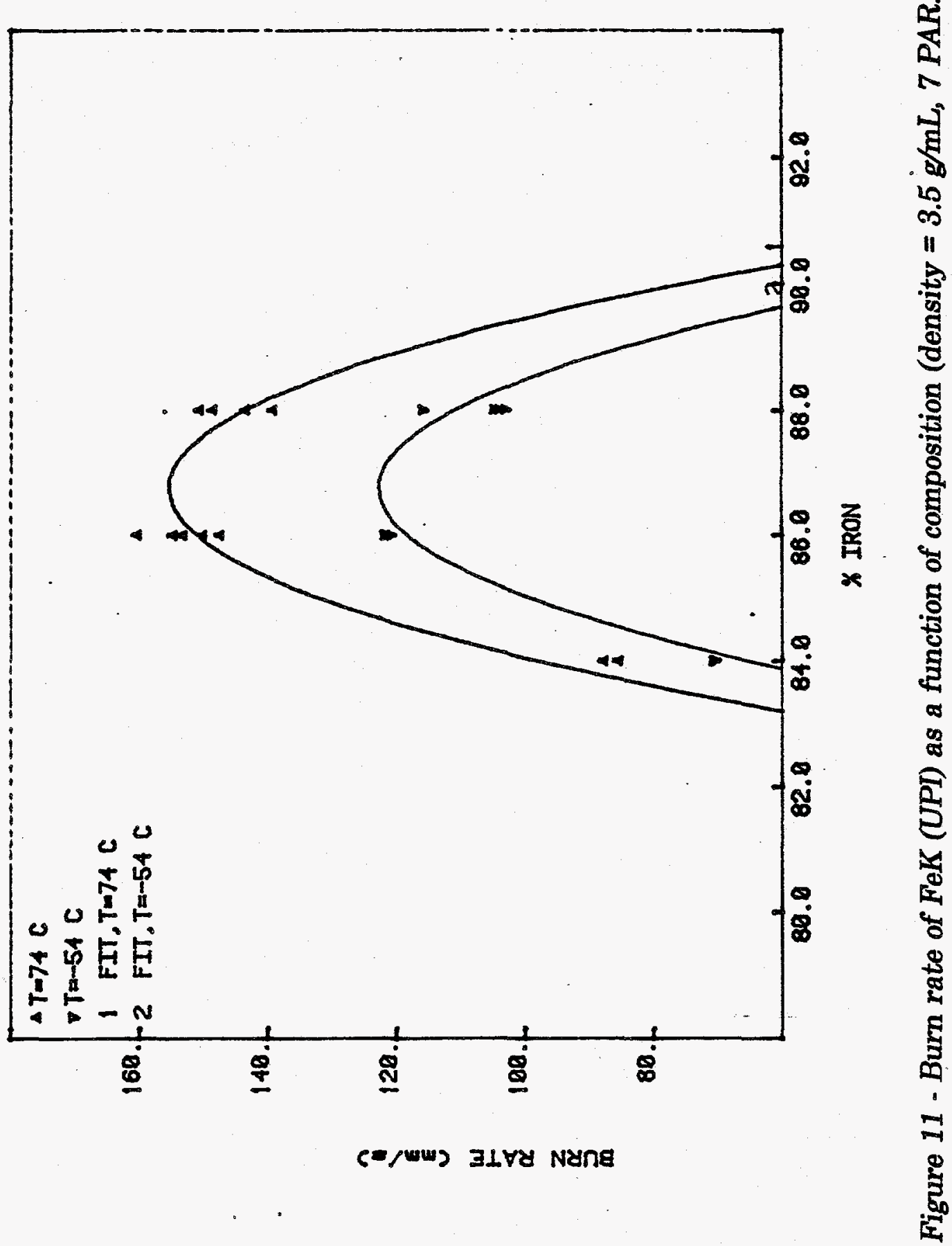




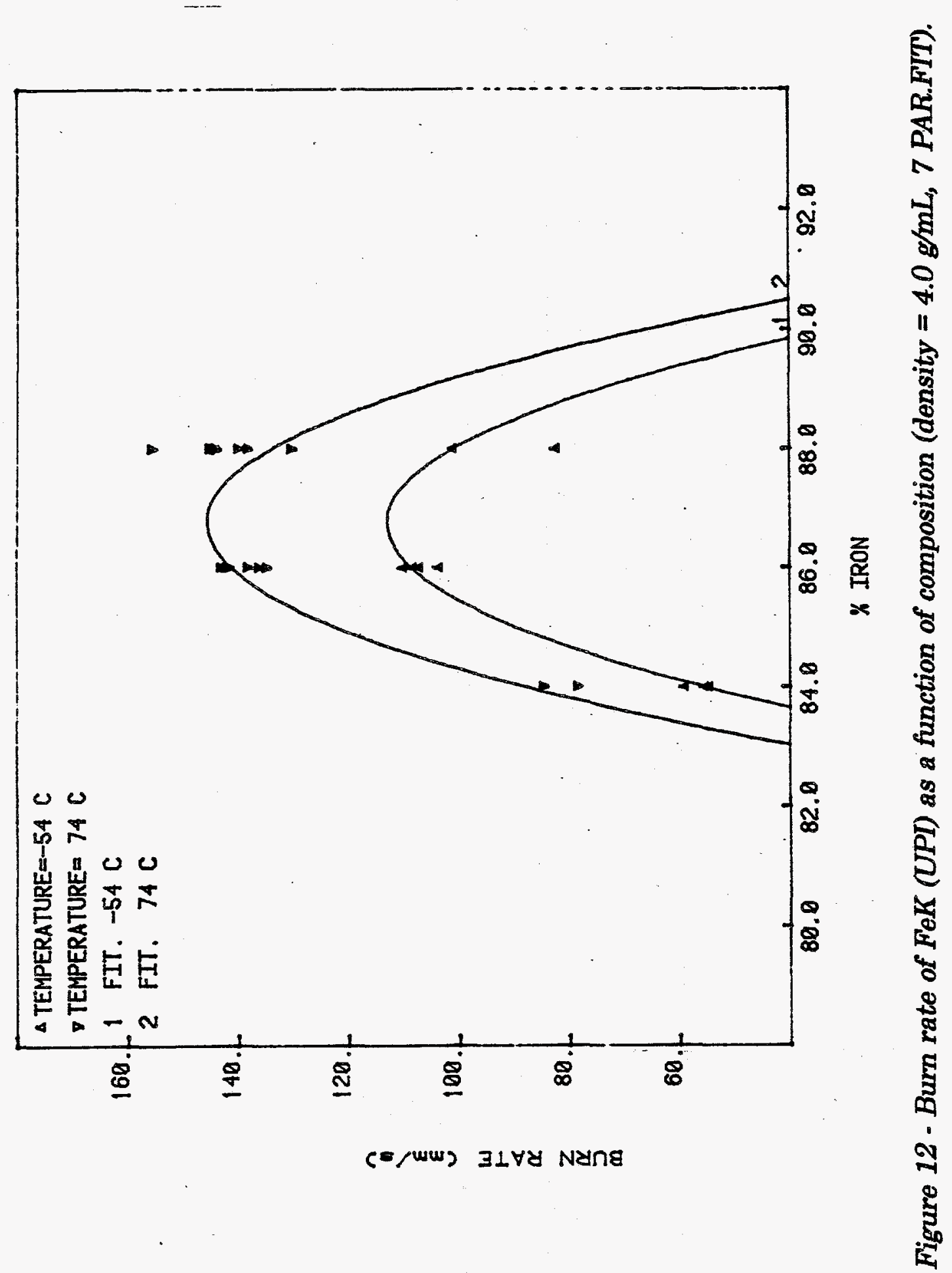




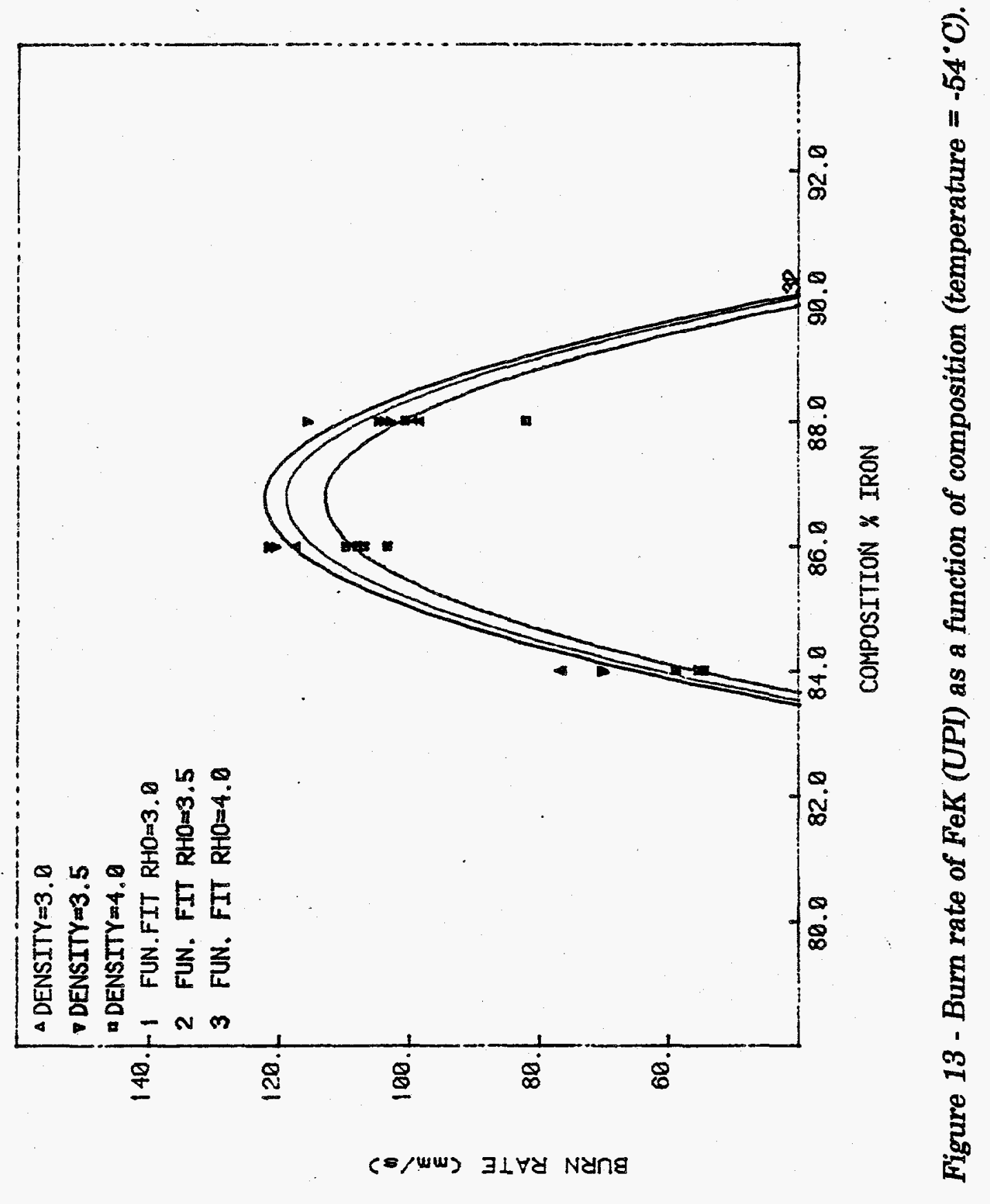




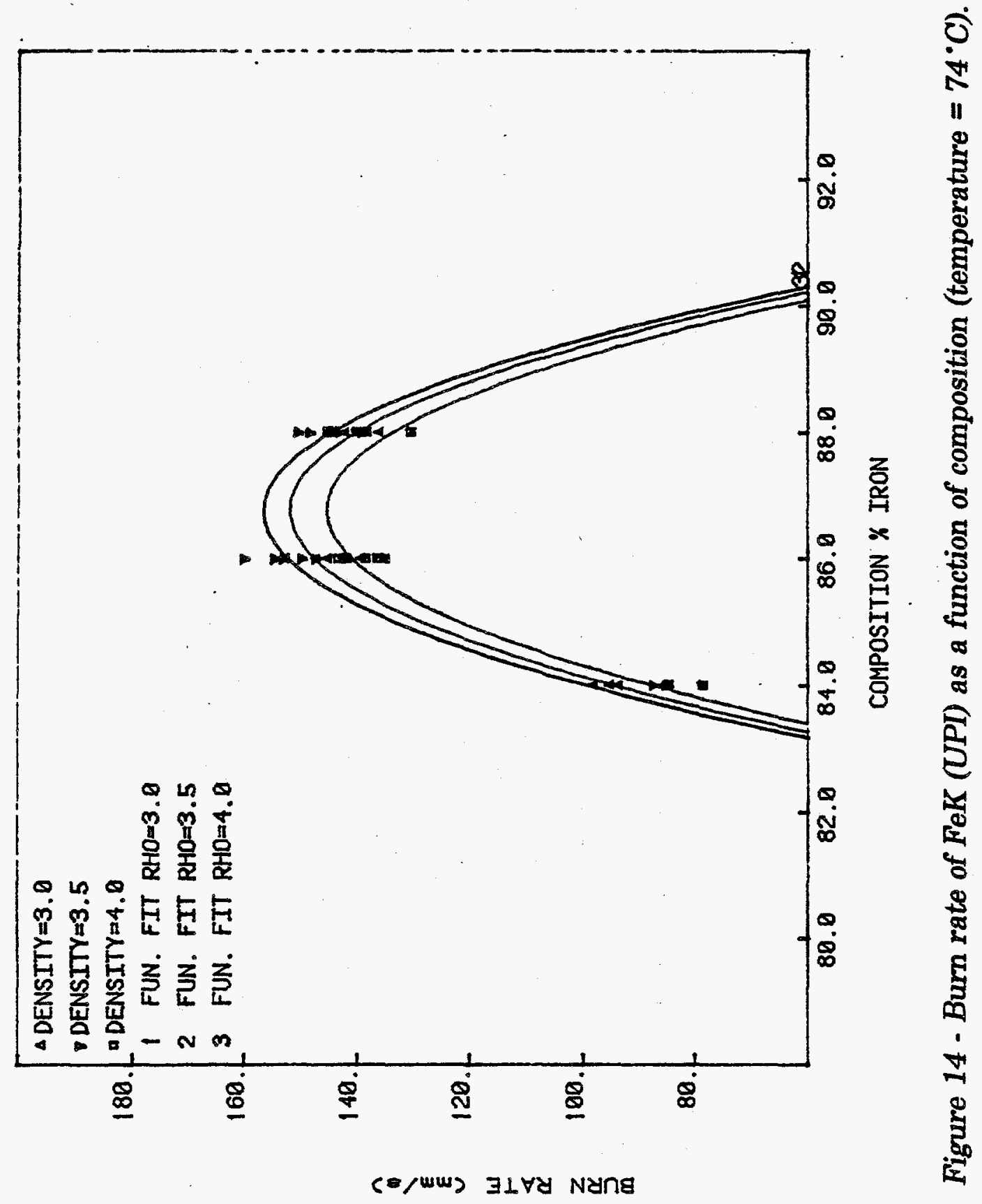




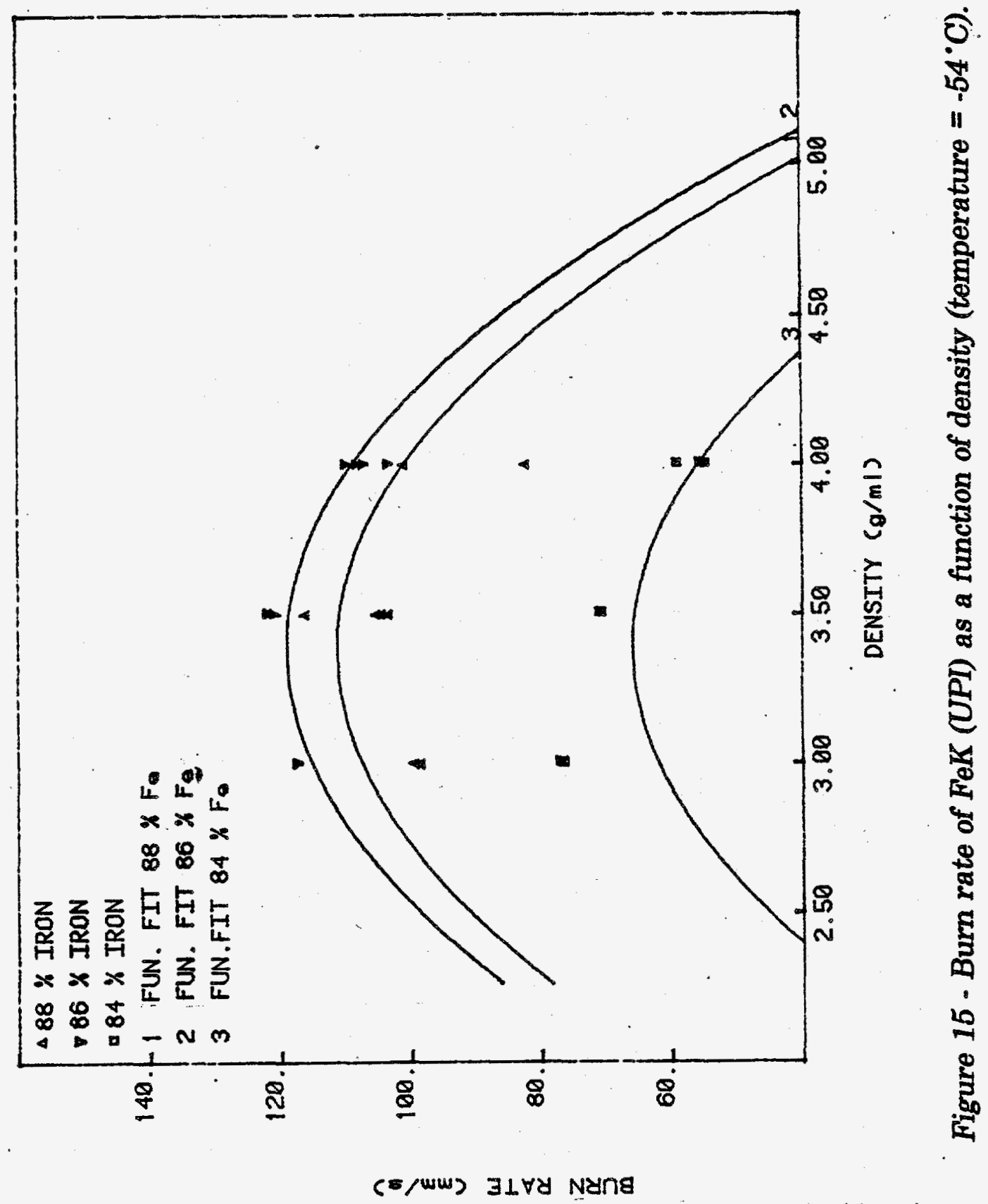




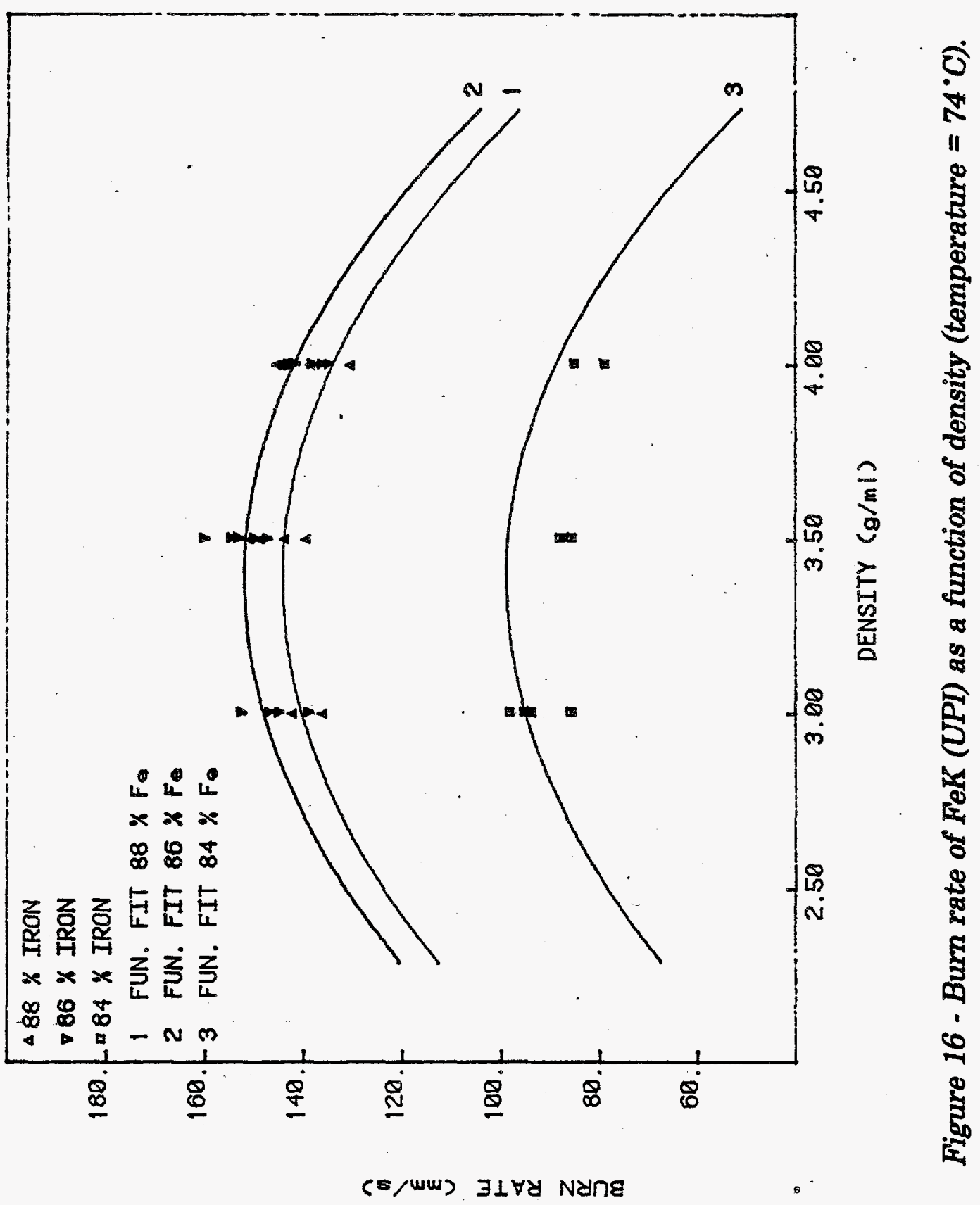


complicating the form of the fitting equation, but the convoluted plots obtained hardly justify the small improvements in fit.

A third set of burn rate determinations were measured on pellets prepared from several Iron / Potassium Perchlorate blends obtained from Eagle Pitcher Corporation. These blends were chosen to determine the effect of iron particle size (surface area) on burn rate, in addition to the effect of the parameters previously discussed. Most of the measurements covered the density range from 3 to $4 \mathrm{~g} / \mathrm{mL}$, while a select group of measurements covered the extended range 2.5 to $5 \mathrm{~g} / \mathrm{mL}$. The pellet pre-ignition temperature range covered was $-54^{\circ} \mathrm{C}$ to $140^{\circ} \mathrm{C}$, a larger range than that for previous cases. The composition range covered was, as in the previous cases, 84 to $88 \%$ iron. Both $\mathrm{Fe} / \mathrm{KClO}_{4}$ composition and particle size of the iron powder in the E-P blends were used to control the powder burn rates of these blends. The particle size data for the iron powder in these blends were obtained as a particle size distribution.

The data were presented as percent iron particles greater than given values versus particle size, as shown graphically in Figure 17. This presentation does not easily lend itself to correlations with burn rate data, since a single value variable is not directly available. Either the average particle size or the integrated surface area may be calculated for the purpose of correlation. Computation of surface area was chosen for the correlation.

The surface area value used for correlation purposes was obtained by assuming a spherical particle geometry, computing the spherical surface areas, and integrating over all particles. The surface area values obtained were thus relative values relating the surface areas of the various iron component materials in the blends. No measured values for the surface areas of the iron materials were available. These calculated surface areas, referred to as surface functions, are shown in Table 2 along with the average particle size, the blend ratio $\left(\mathrm{Fe} / \mathrm{KClO}_{4}\right)$, the nominal open train powder burn rate, and the Sandia National Laboratory blend number.

The variation of burn rate with density for the above blends at several temperatures $\left(-54^{\circ}, 25^{\circ}, 74^{\circ}\right.$, and $\left.140^{\circ} \mathrm{C}\right)$ is shown in Figures 18 through 25. Each figure represents individual values of $\mathrm{Fe} / \mathrm{KClO}_{4}$ ratios and surface 


\section{Table 2 - SURFACE FUNCTIONS}

\begin{tabular}{|c|cc|c|c|}
\hline \multicolumn{5}{c|}{ Table 2 - SURFACE FUNCTIONS } \\
$\begin{array}{c}\text { BLEND } \\
\#\end{array}$ & $\begin{array}{c}\text { Fe/KClO } \\
\text { RATIO }\end{array}$ & $\begin{array}{c}\text { SURFACE } \\
\text { FUNCTION }\end{array}$ & $\begin{array}{c}\text { Fe AVG. } \\
\text { MICRONS }\end{array}$ & $\begin{array}{c}\text { NOMINAL } \\
\text { RATE }\end{array}$ \\
\hline PBF- & RATIO & & \\
\hline 0395 & $88 / 12$ & 23.7 & 10.5 & 4.56 \\
0396 & $86 / 14$ & 23.7 & 10.5 & 2.71 \\
0400 & $86 / 14$ & 14.9 & 18.6 & 3.9 \\
0401 & $84 / 16$ & 13.7 & 20.3 & 4.28 \\
0402 & $84 / 16$ & 24.07 & 10.5 & 5.1 \\
0404 & $84 / 16$ & 9.8 & 37.3 & 3.02 \\
0405 & $86 / 14$ & 11.0 & 31.3 & 2.55 \\
0406 & $88 / 12$ & 28.0 & 8.8 & 5.87 \\
\hline
\end{tabular}

functions. The points represent individual measurements of burn rates. The line plots are obtained from individual, three-parameter, polynomial least square fits of burn rate as a function of density for each set of remaining independent variables. Figures 18 and 19 are somewhat misleading on first inspection because not enough variation in density values are shown to indicate the curvature and the maxima, which can be observed in the figures following (20-25).

In several figures $(23,24)$ the density varies over the range 2.5 to $5.0 \mathrm{~g} / \mathrm{mL}$. These figures represent the only sets of data over this wide range of density values primarily because of the difficulty in pressing pellets at the extreme low and high ends of the range. At the low end of the range $(2.5 \mathrm{~g} / \mathrm{mL})$ handling of the pellets which were available was very difficult without breaking the pellets. Pellets in the 4.5 to $5.0 \mathrm{~g} / \mathrm{mL}$ density range were extremely difficult to ignite. In general the maximum values in burn rate appear at densities of approximately 3.2 to $3.4 \mathrm{~g} / \mathrm{mL}$. In these figures the burn rate varies directly with temperature over the full temperature range of the measurements. These figures cover the full range of all of the variables studied. The rather consistent variation of burn rate with density and 
temperature in Figures 18 through 25 suggests the possibility of fitting burn rate to some linear combination of density and temperature over the range of measurements.

The general trend in burn rate with surface function is shown in Figures ' 26 through 32 for the several values of $\mathrm{Fe} / \mathrm{KClO}_{4}$ ratio. The trend in burn rate with surface function for the $88 / 12 \mathrm{Fe} / \mathrm{KClO}_{4}$ blend is different than that for the other two blends (86/14 and 84/16). This trend is consistent over the density and temperature ranges for this blend. The range of surface function values available for this blend is not, however, the same as that for the other two blends (86/14 and 84/16). The high surface function blends for $86 / 14$ and $84 / 16$ ratios and the low surface function blends for the $88 / 12$ ratio are missing.

It appears that the high ratio blend behaves differently than the lower ratio blends. Without burn rate data for the extended values this discrepancy cannot be resolved. The inverse plots are shown in Figures 33 through 36 where burn rate is plotted as a function of blend ratios at several values of surface function, temperature and density. In Figures 33 and 35 the density is $3.5 \mathrm{~g} / \mathrm{mL}$ and in 34 and 36 it is $4.0 \mathrm{~g} / \mathrm{mL}$. No set of pellets covering a wide enough range of $\mathrm{Fe} / \mathrm{KClO}_{4}$ compositions made from iron of a single surface area was available to produce any of these curves. Each curve is made up of burn rate data from blends of similar surface area iron. The burn rates obtained for the high values of surface function are not shown in these figures but would lie well off the curves. The only blend containing the high surface iron was $\mathrm{Fe} / \mathrm{KClO}_{4}=88 / 12$.

As with the UPI burn rate data, an attempt was made to obtain a useful numeric representation of the E-P burn rate data using various empirical equations. As noted previously, the $88 / 12 \mathrm{E}-\mathrm{P}$ blends containing high surface area iron behaved differently than the other blends. This unusual behavior made fitting the data difficult, and a simple linear combination of second degree terms did not produce as good a fit as that obtained for the UPI blends. Various combinations of power and cross terms were applied, usually producing rather convoluted fits. A twelve-term equation was finally found to be acceptable without a great deal of exaggerated convolution exhibited. 


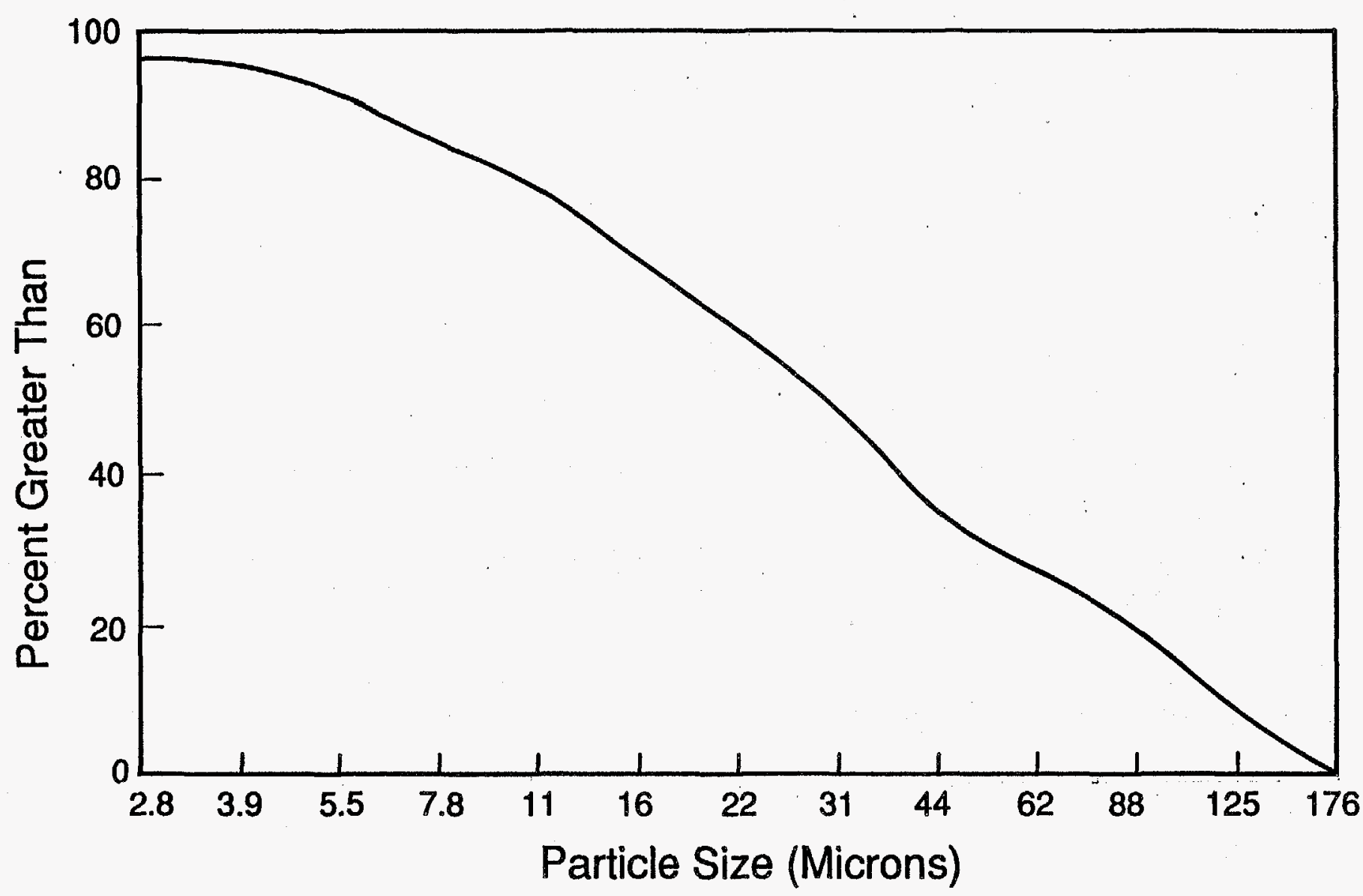

Bulk Density: 26.7 g/cu.in.

Figure 17 - Percent iron particles greater than given values versus particle size. 


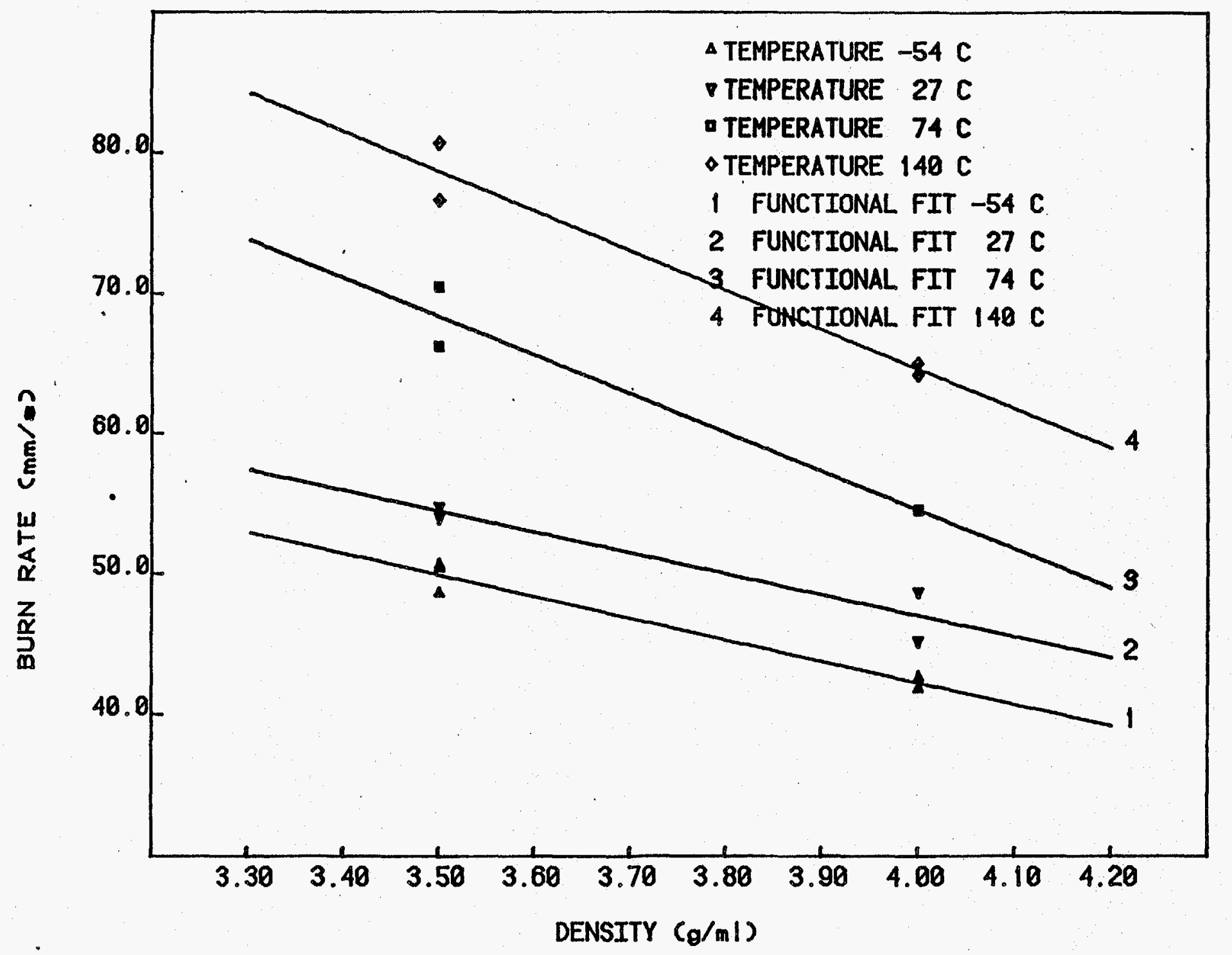

Figure 18 - Burn rate of $\mathrm{FeK}(84 / 16, \mathrm{E}-\mathrm{P}$ ) as a function of density (nom rate $77 \mathrm{~mm} / \mathrm{s}, \mathrm{SF}=9.8$ ). 


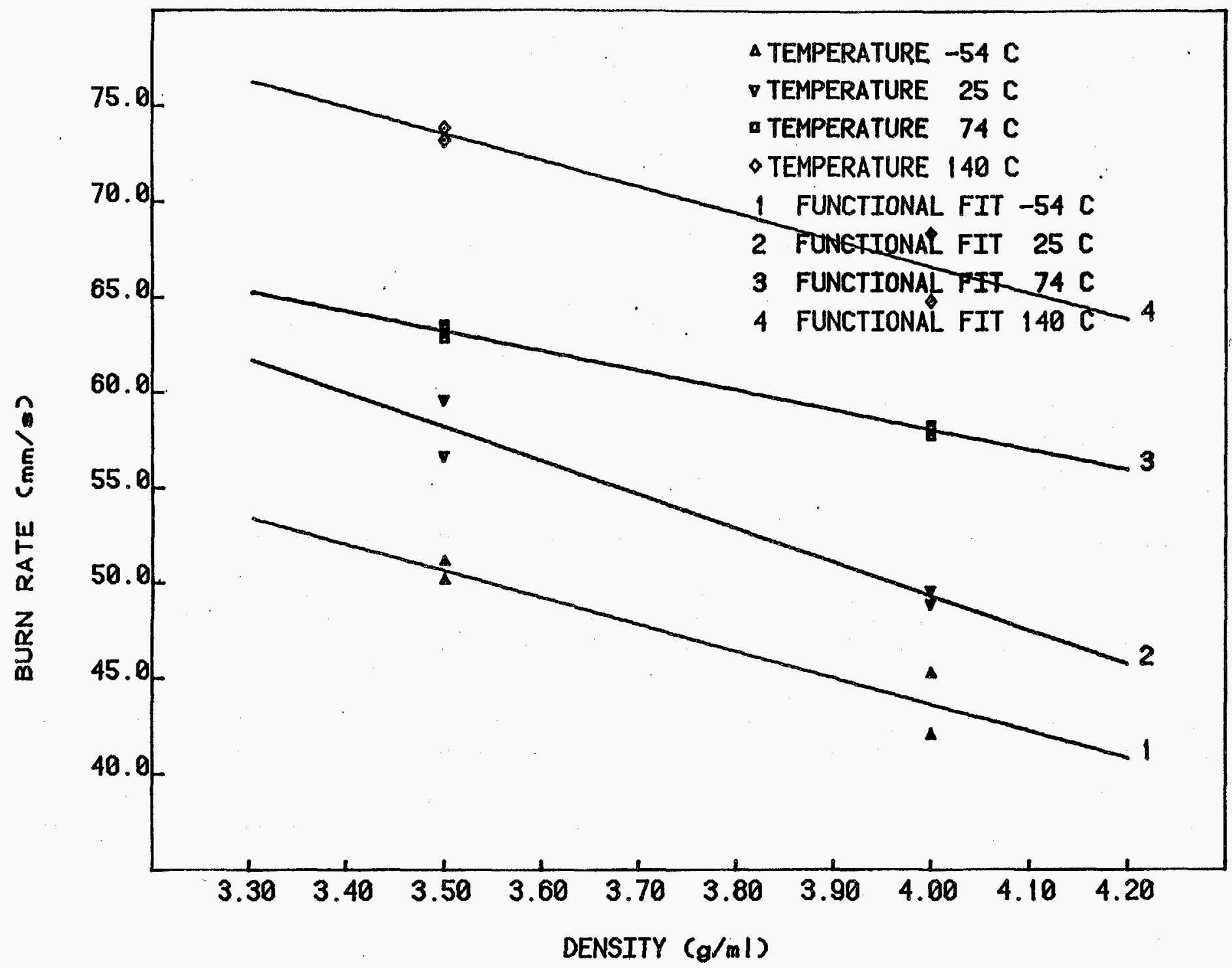

Figure 19 - Burn rate of FeK $(86 / 14, E-P)$ as a function of density (nom rate $65 \mathrm{~mm} / \mathrm{s}, S F=11.0$ ). 


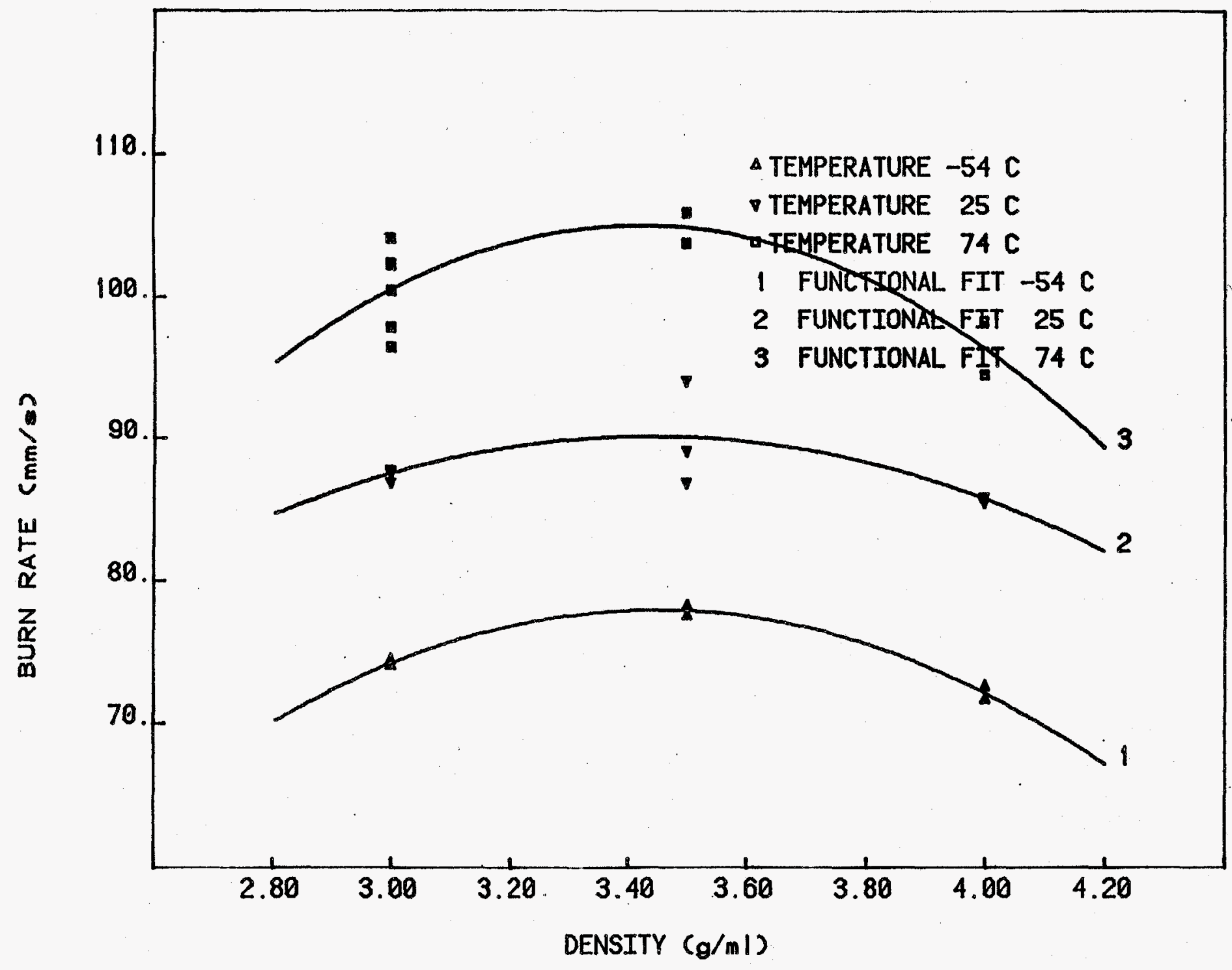

$\stackrel{\omega}{\sim}$ Figure 20 - Burn rate of $F e K(88 / 12, E-P)$ as a function of density (nom rate $68.6 \mathrm{~mm} / \mathrm{s}, \mathrm{SF}=14.7$ ). 
W

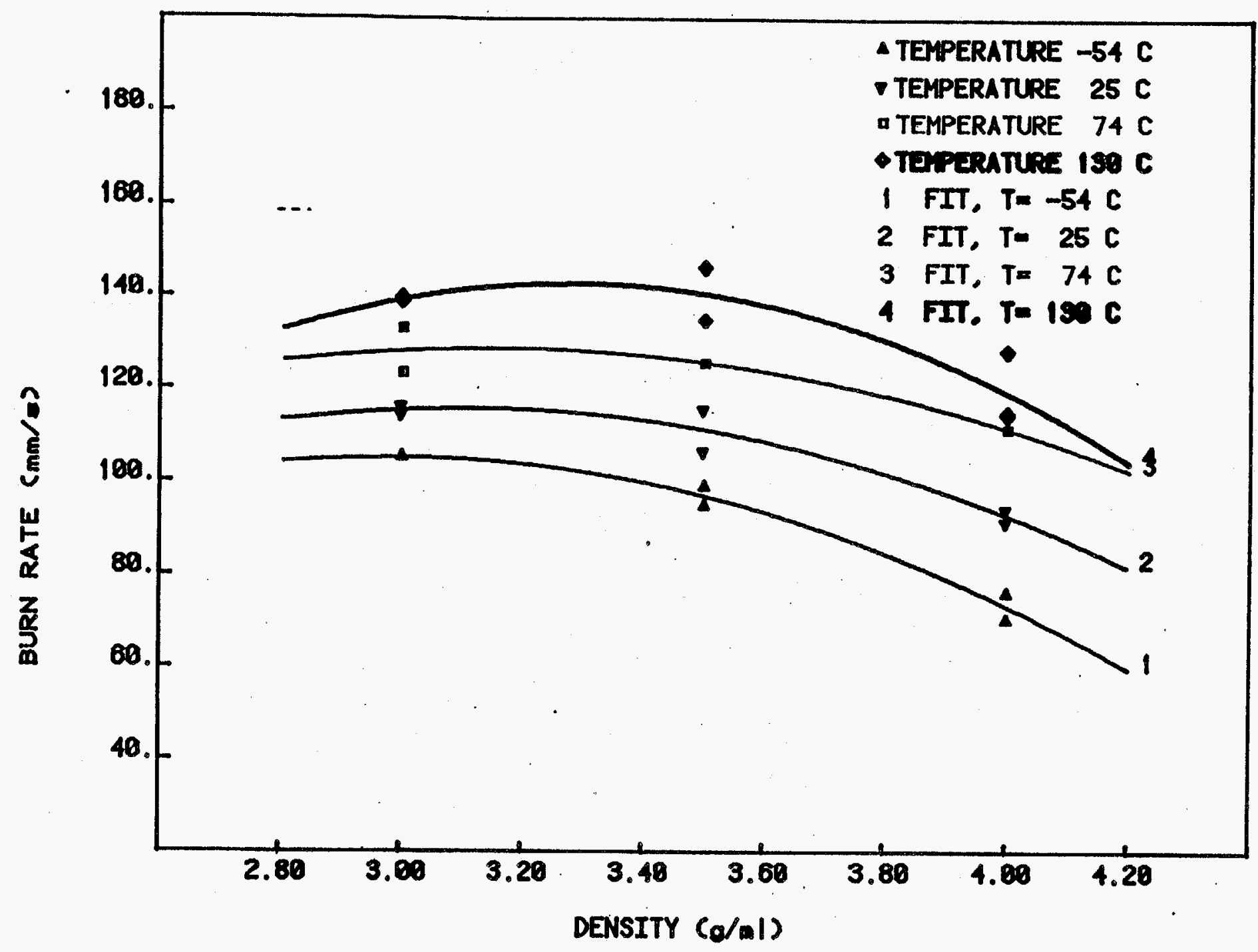

Figure 21 - Burn rate of $\mathrm{FeK}(84 / 16, E-P)$ as a function of density, surface function = 24.07. 


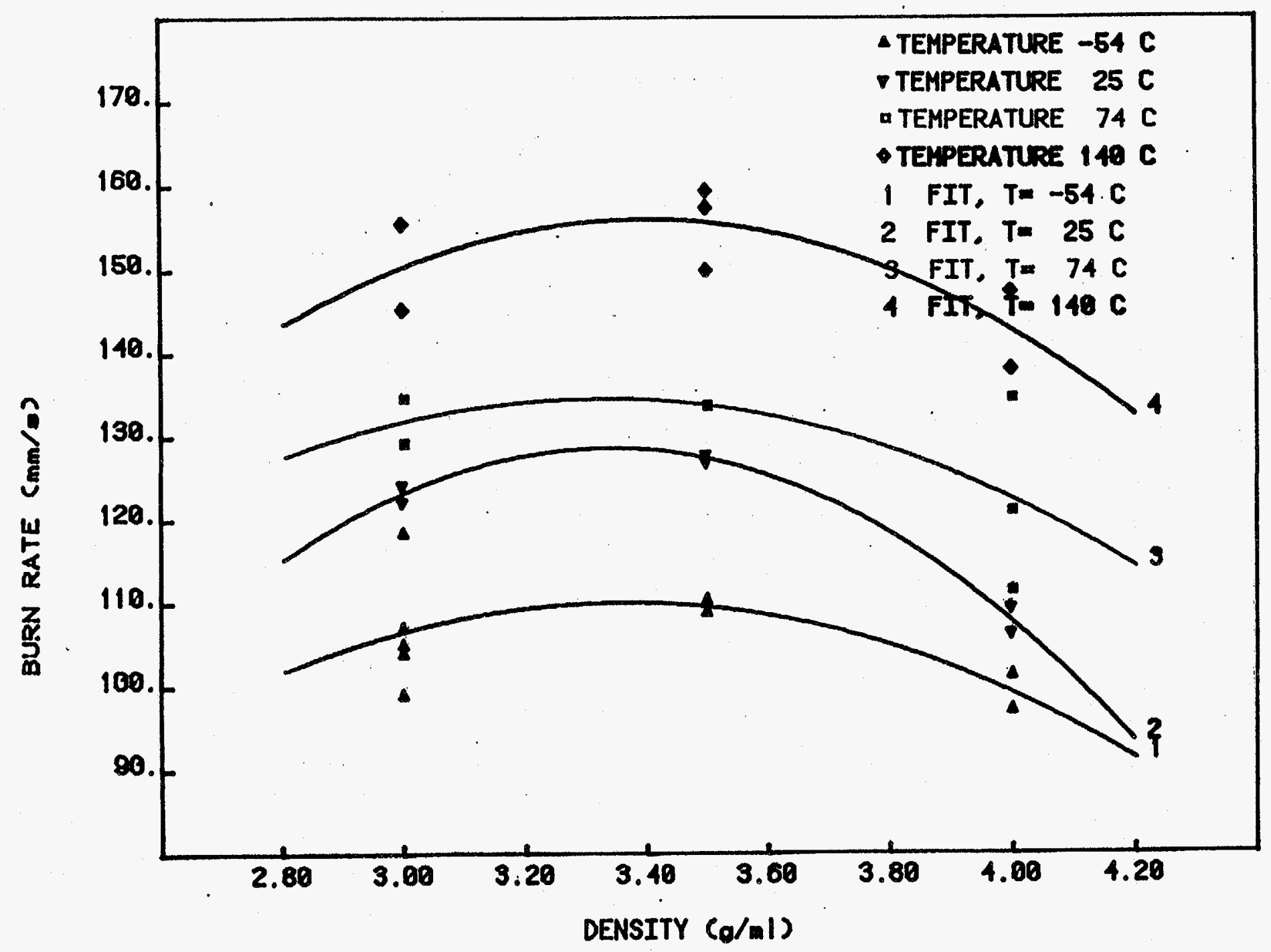

Figure 22 - Burn rate of $\mathrm{FeK}(86 / 14, E-P)$ as a function of density, surface function $=23.7$. 
$\underset{\omega}{\infty}$

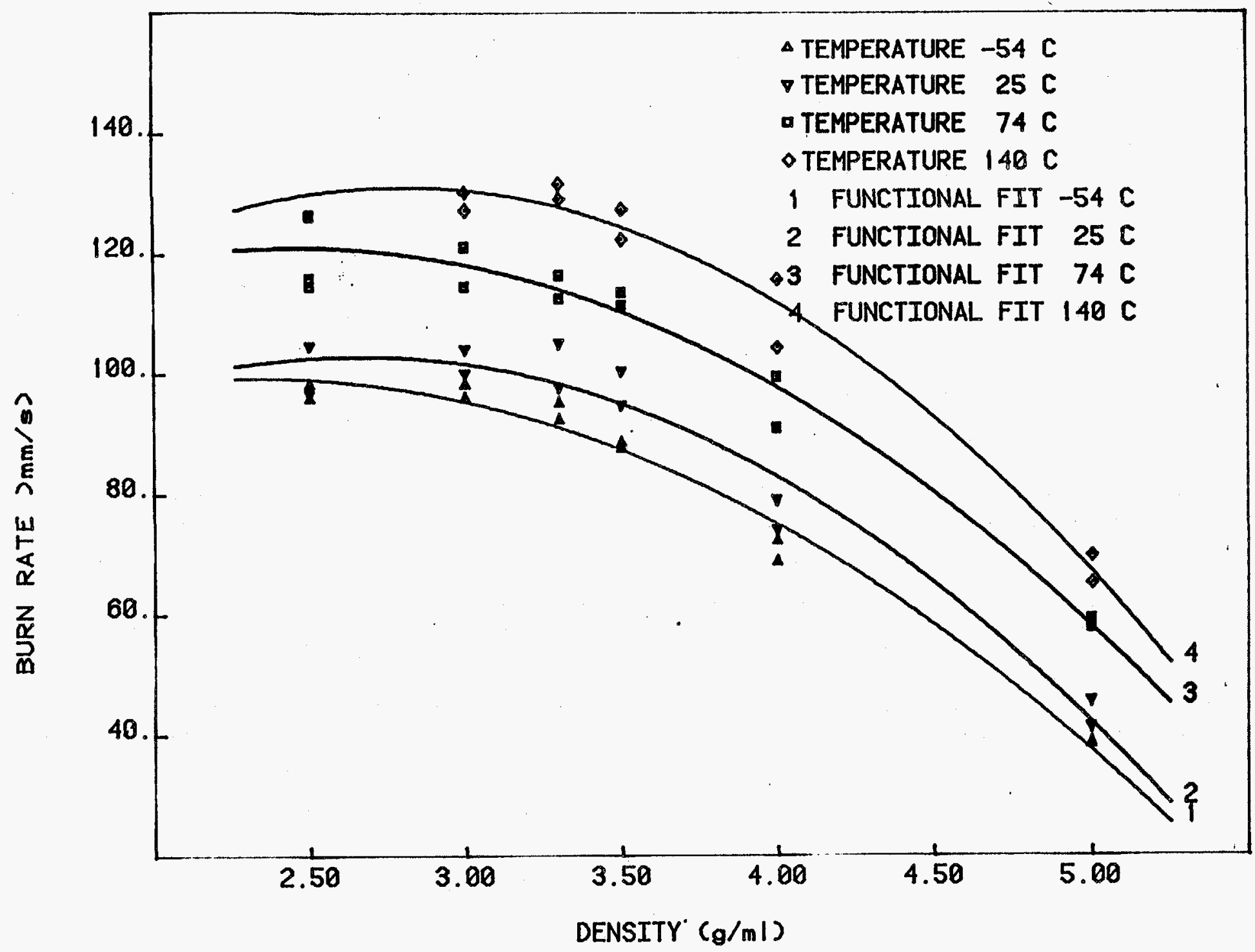

Figure 23 - Burn rate of $\mathrm{FeK}(84 / 16, \mathrm{E}-\mathrm{P}$ ) as a function of density (nom rate $109 \mathrm{~mm} / \mathrm{s}, \mathrm{SF}=13.7$ ). 


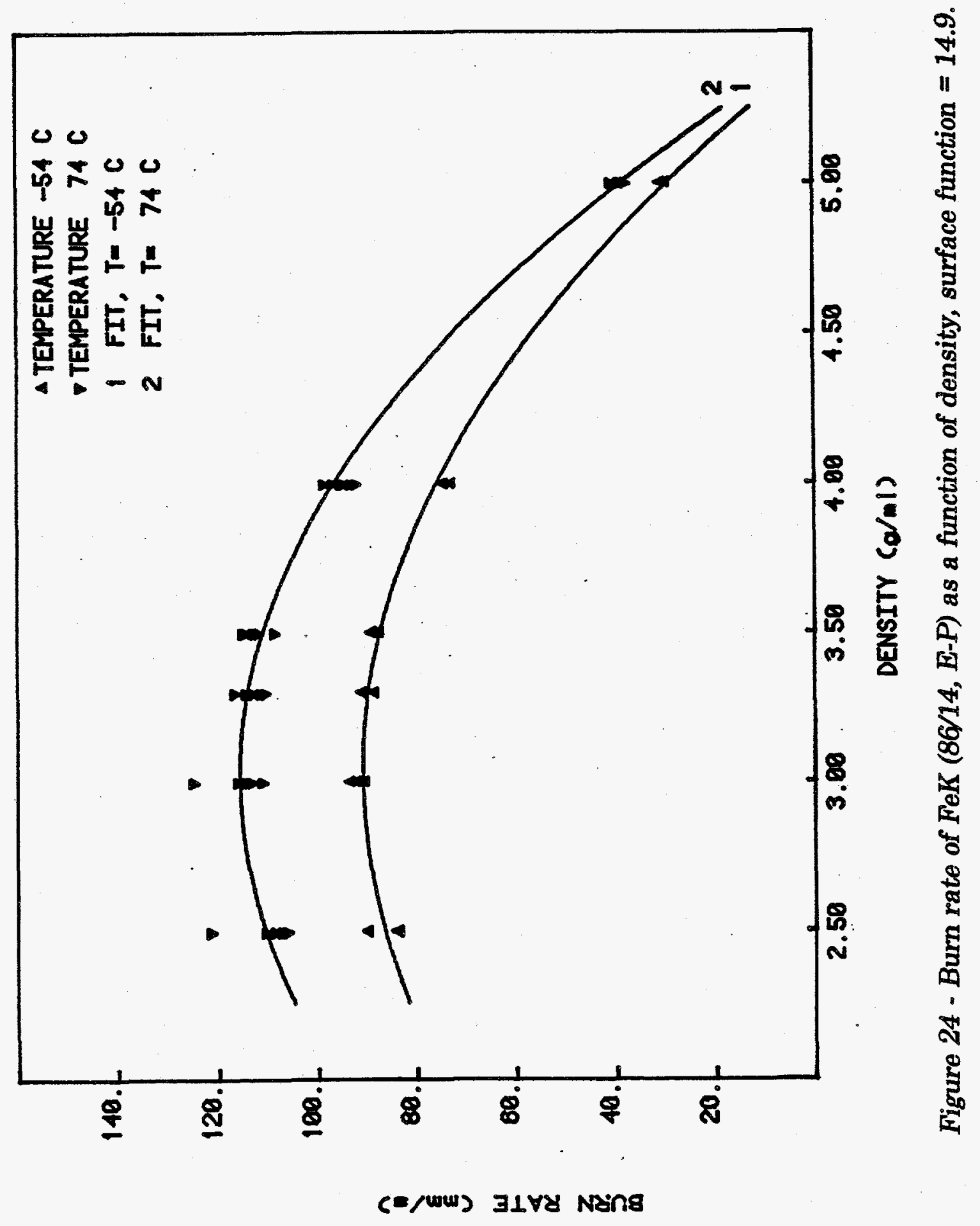




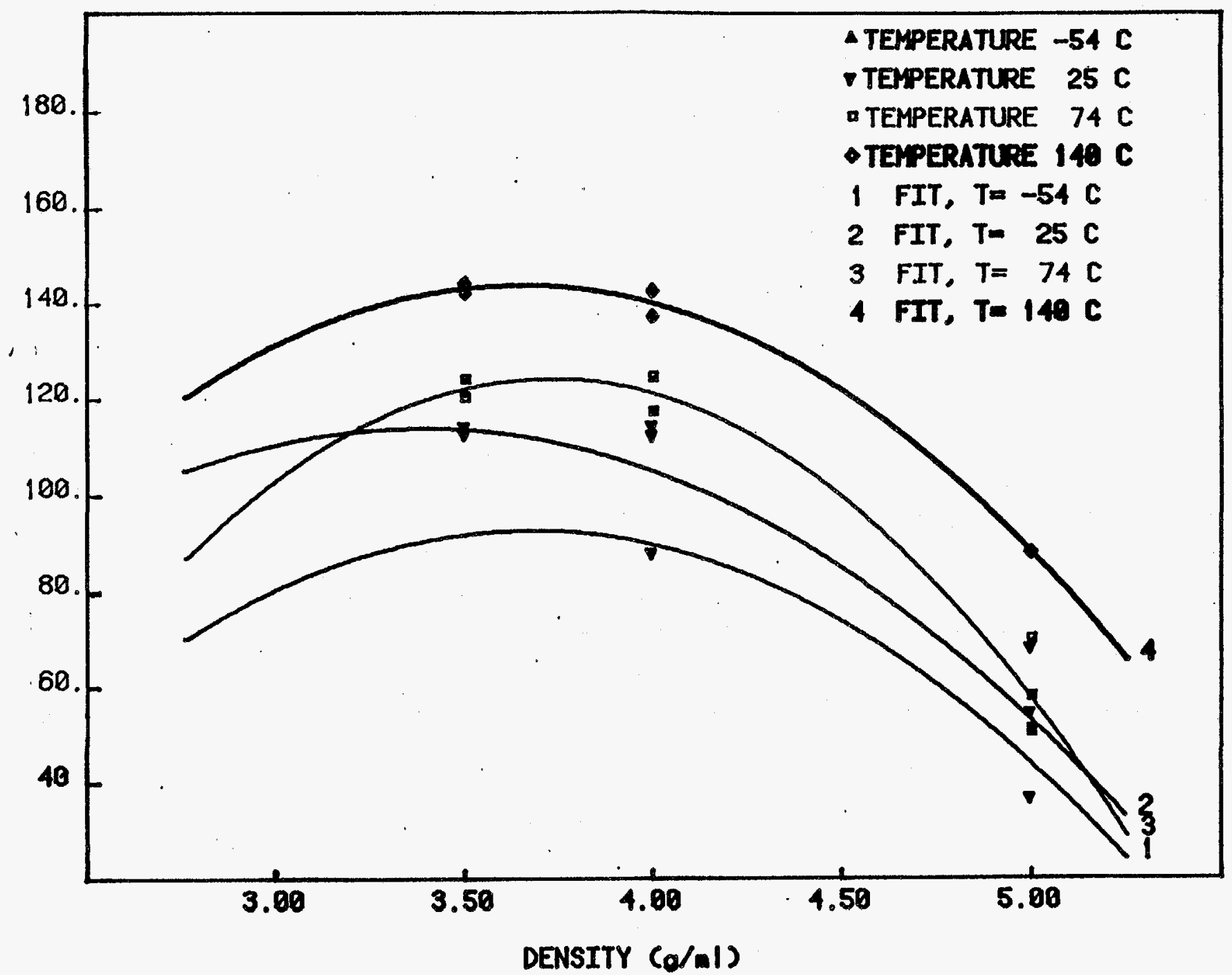

Figure 25 - Burn rate of $\mathrm{FeK}(88 / 12, \mathrm{E}-\mathrm{P})$ as a function of density, surface function $=23.7$. 


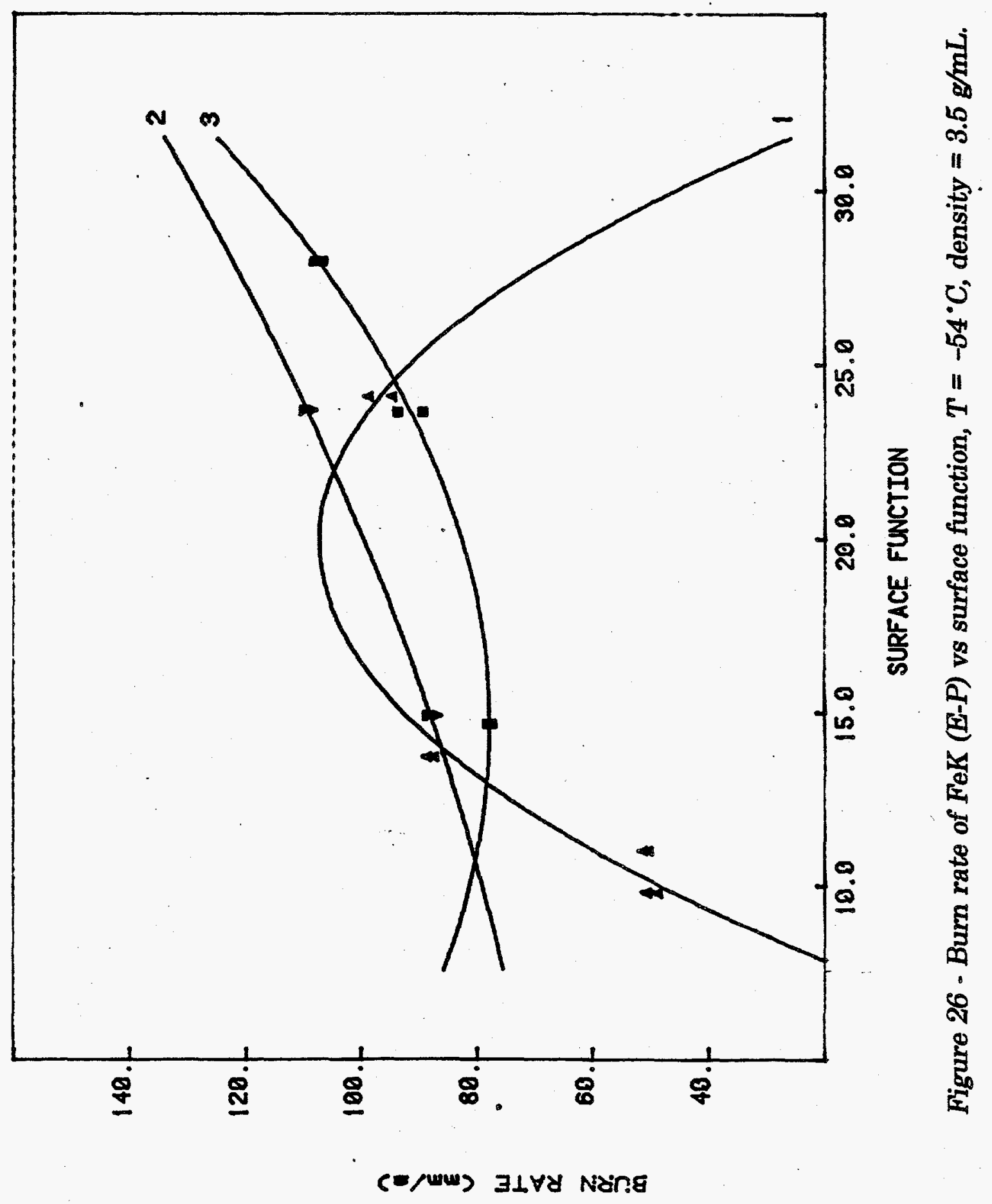




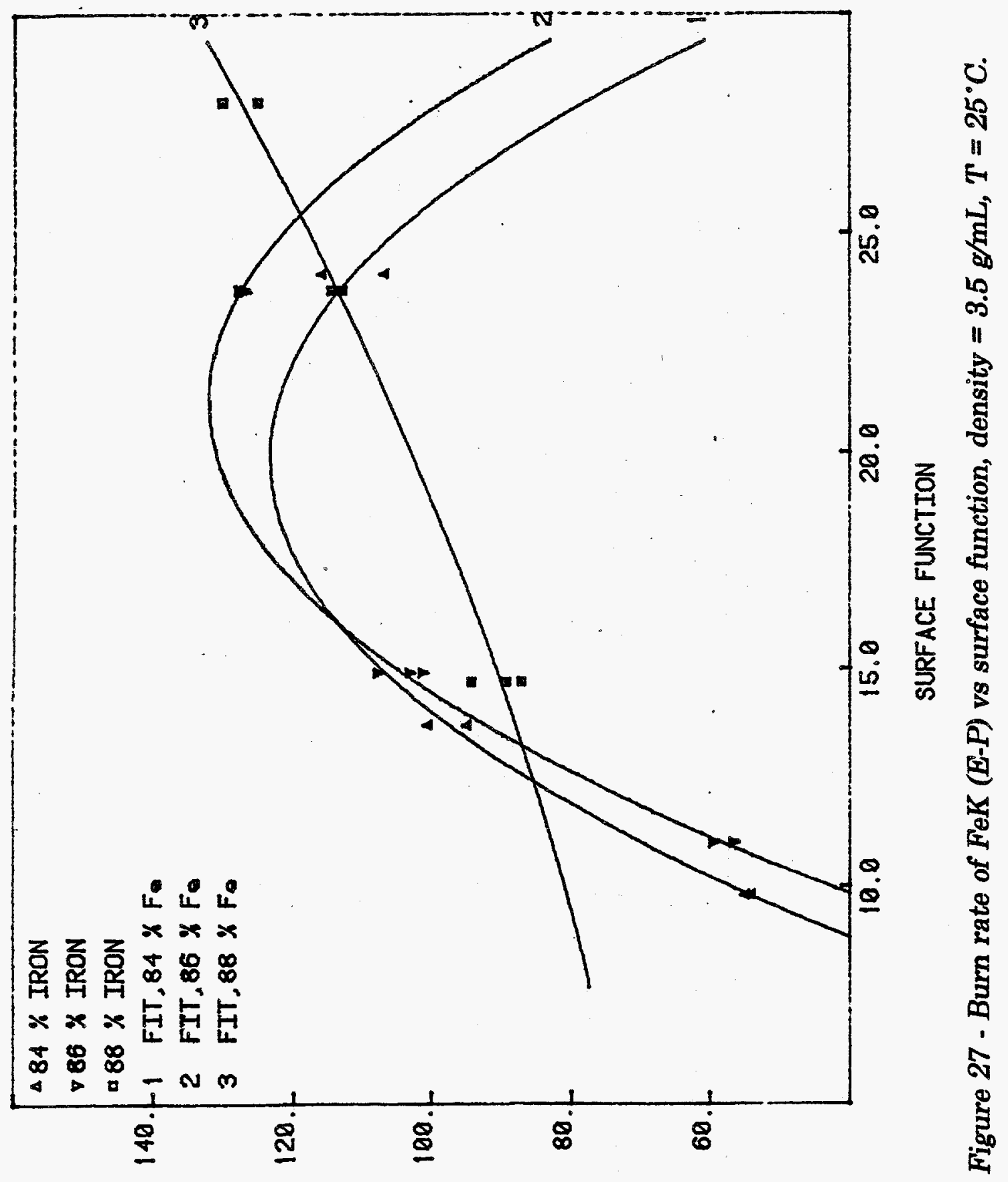

(s/ww) $\exists \perp \forall y$ Nyng 


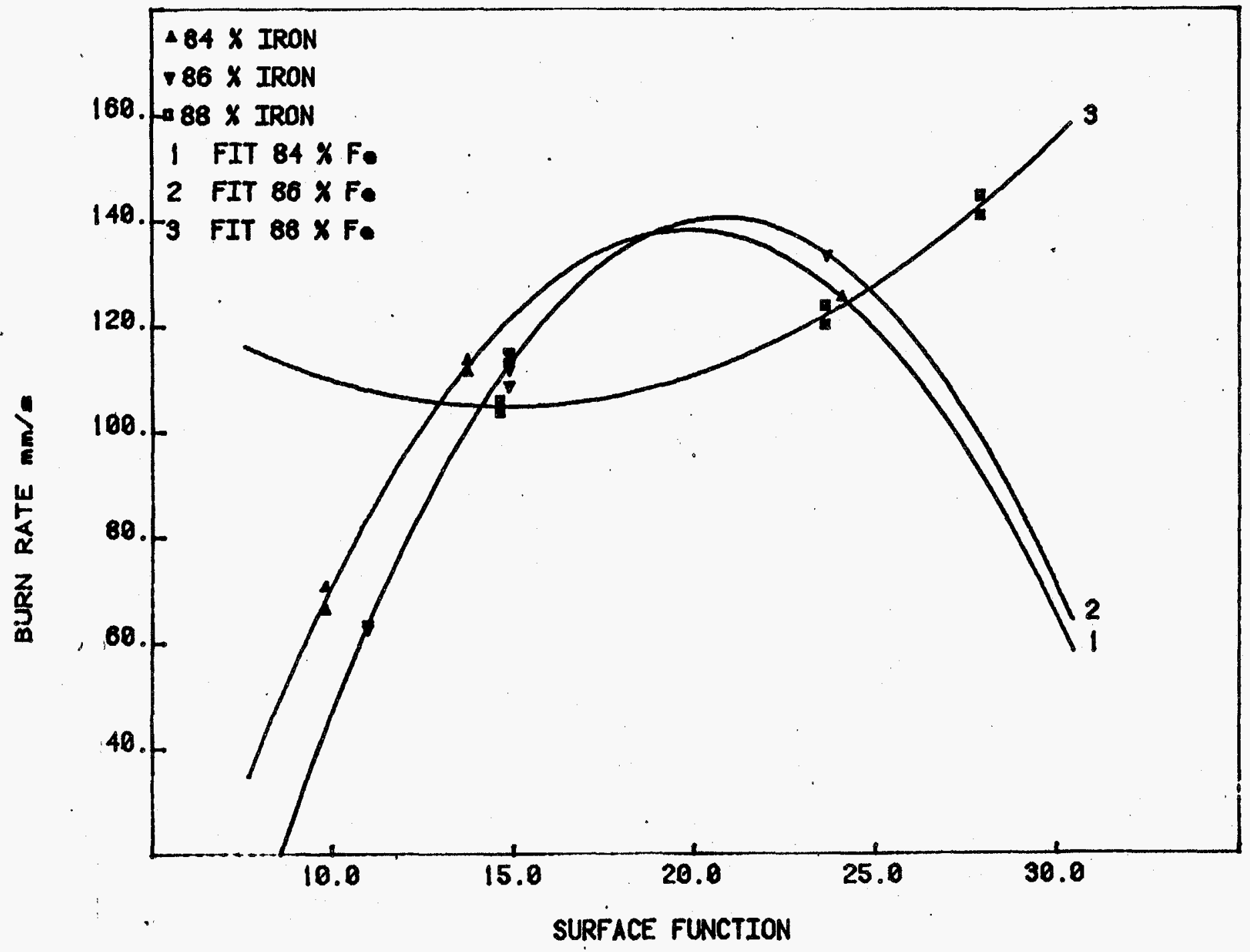

ષ్రు

Figure 28 - Burn rate of $\mathrm{FeK}(\mathrm{E}-\mathrm{P})$ vs surface function, density $=3.5 \mathrm{~g} / \mathrm{mL}, \mathrm{T}=74^{\circ} \mathrm{C}$. 
to

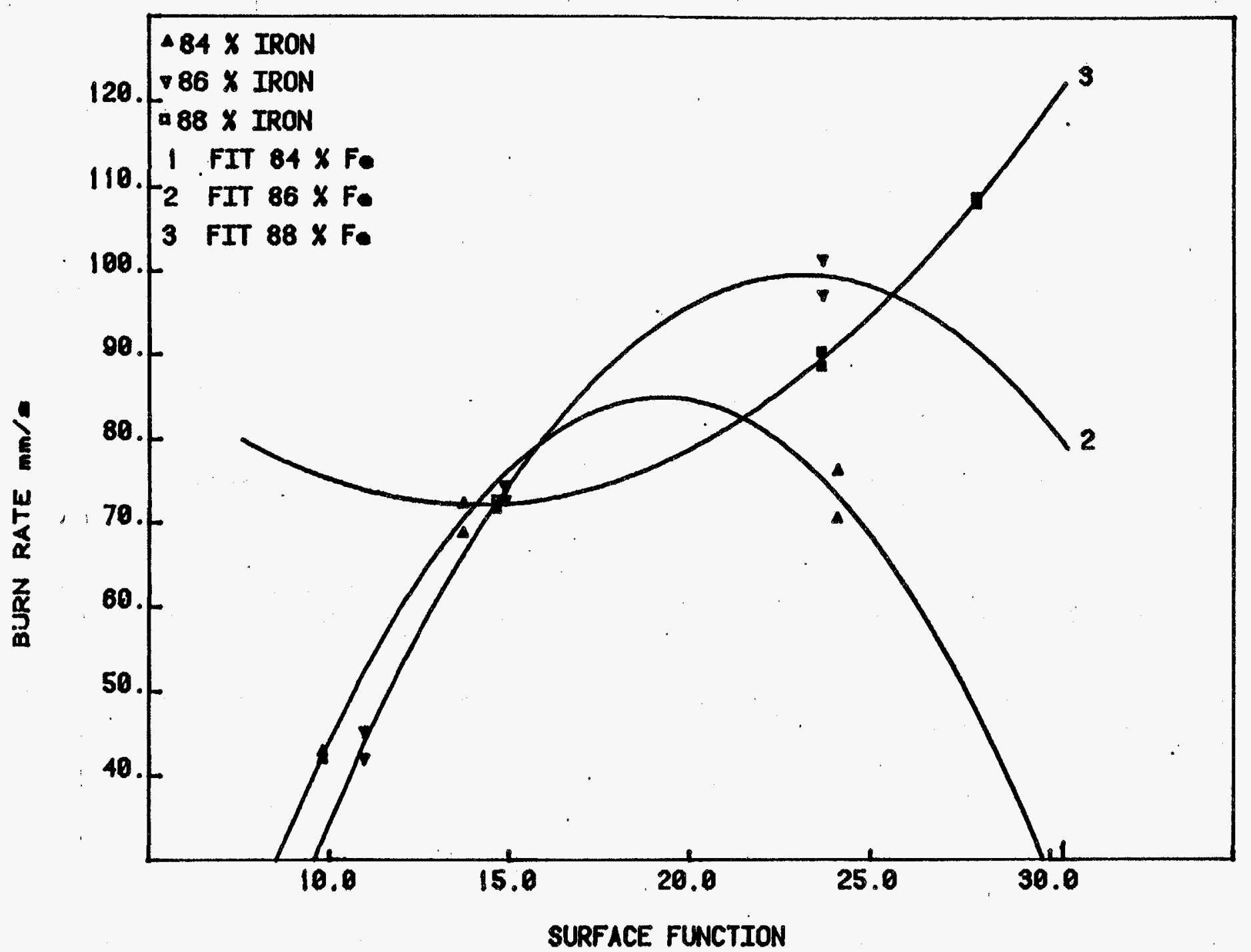

Figure 29 - Burn rate of $\mathrm{FeK}(\mathrm{E}-\mathrm{P})$ vs surface function, density $=4 \mathrm{~g} / \mathrm{mL}, \mathrm{T}=-54^{\circ} \mathrm{C}$. 


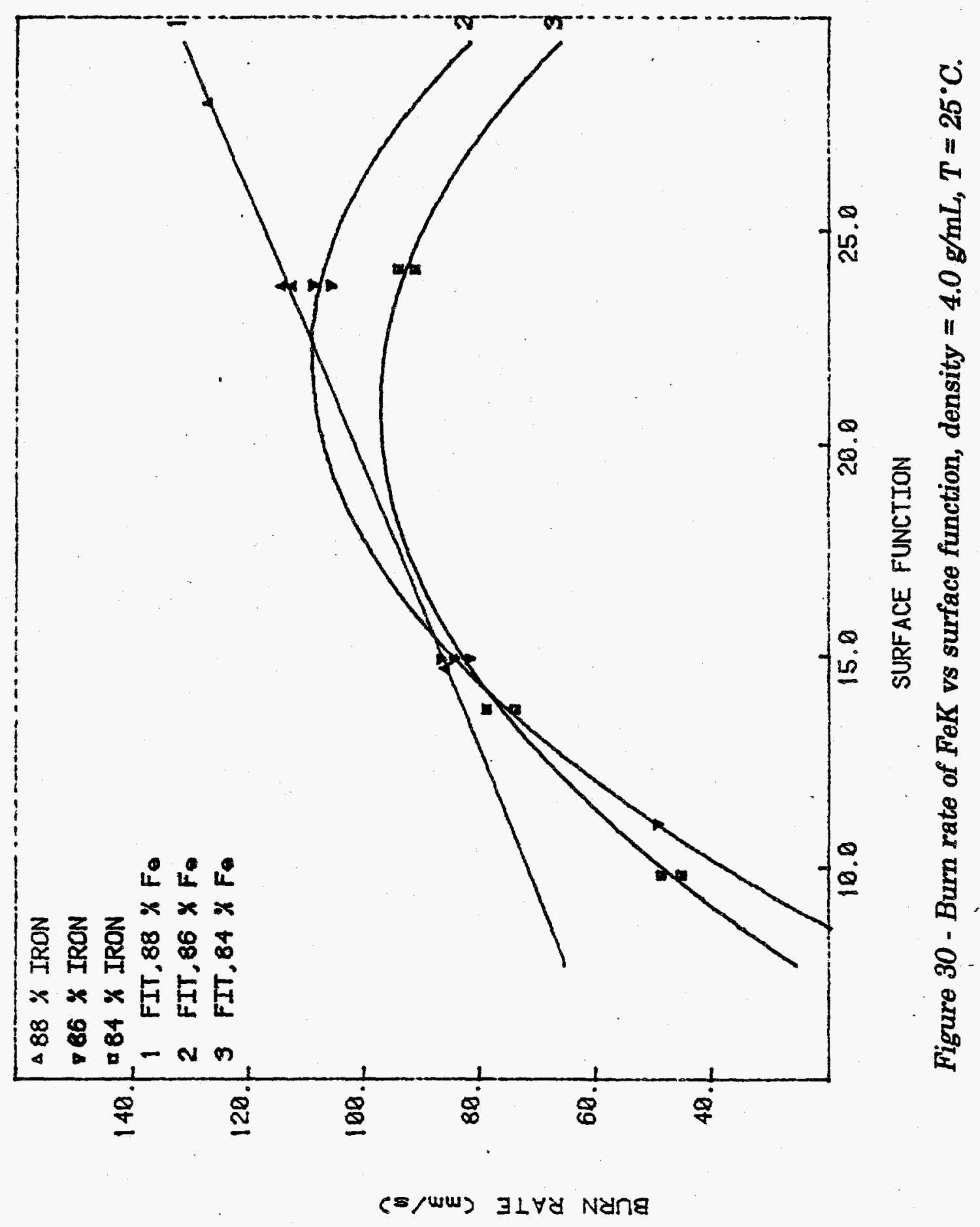


N

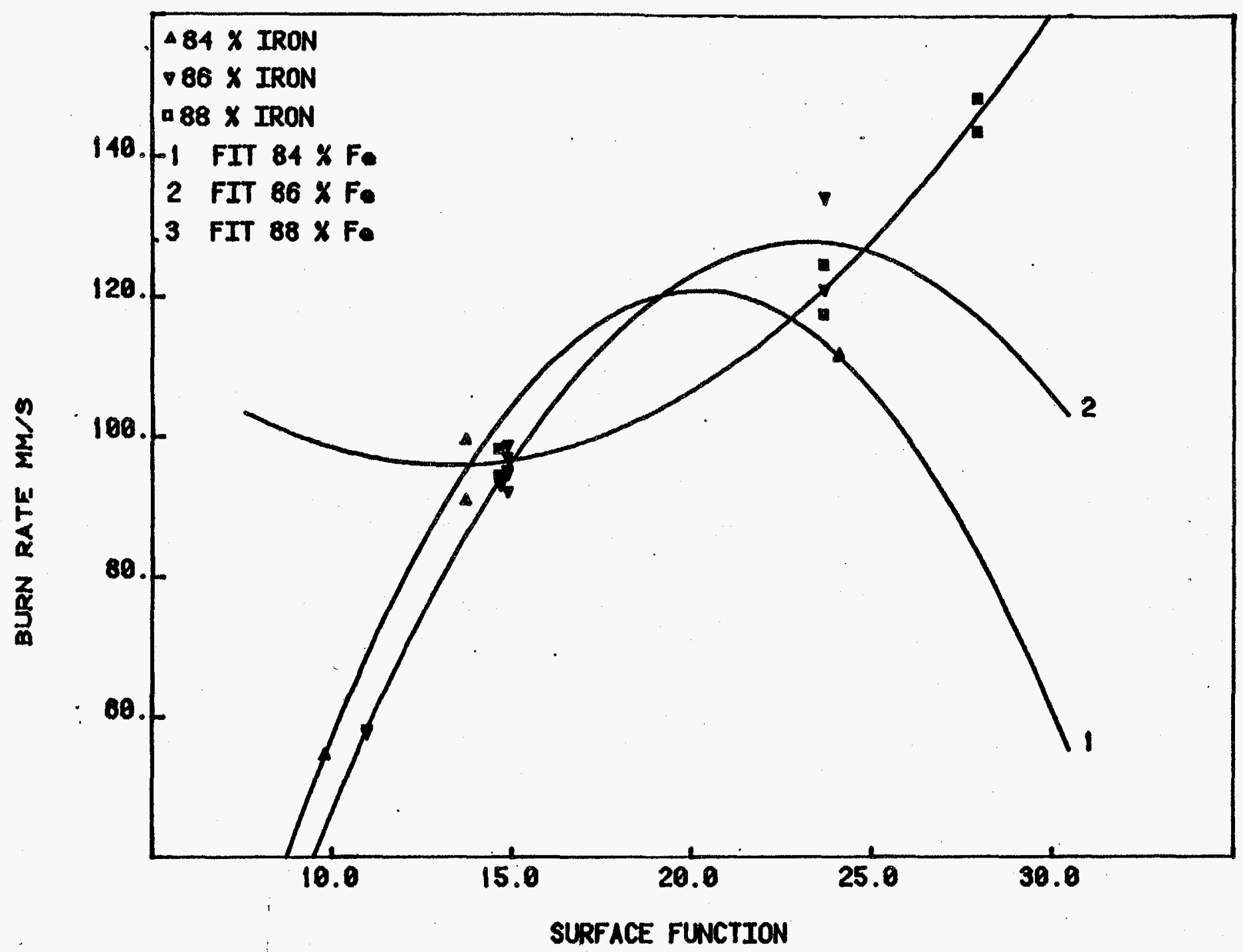

Figure 31 - Burn rate of $\mathrm{FeK}(E-P)$ vs surface function, density $=4.0 \mathrm{~g} / \mathrm{mL}, \mathrm{T}=74^{\circ} \mathrm{C}$. 


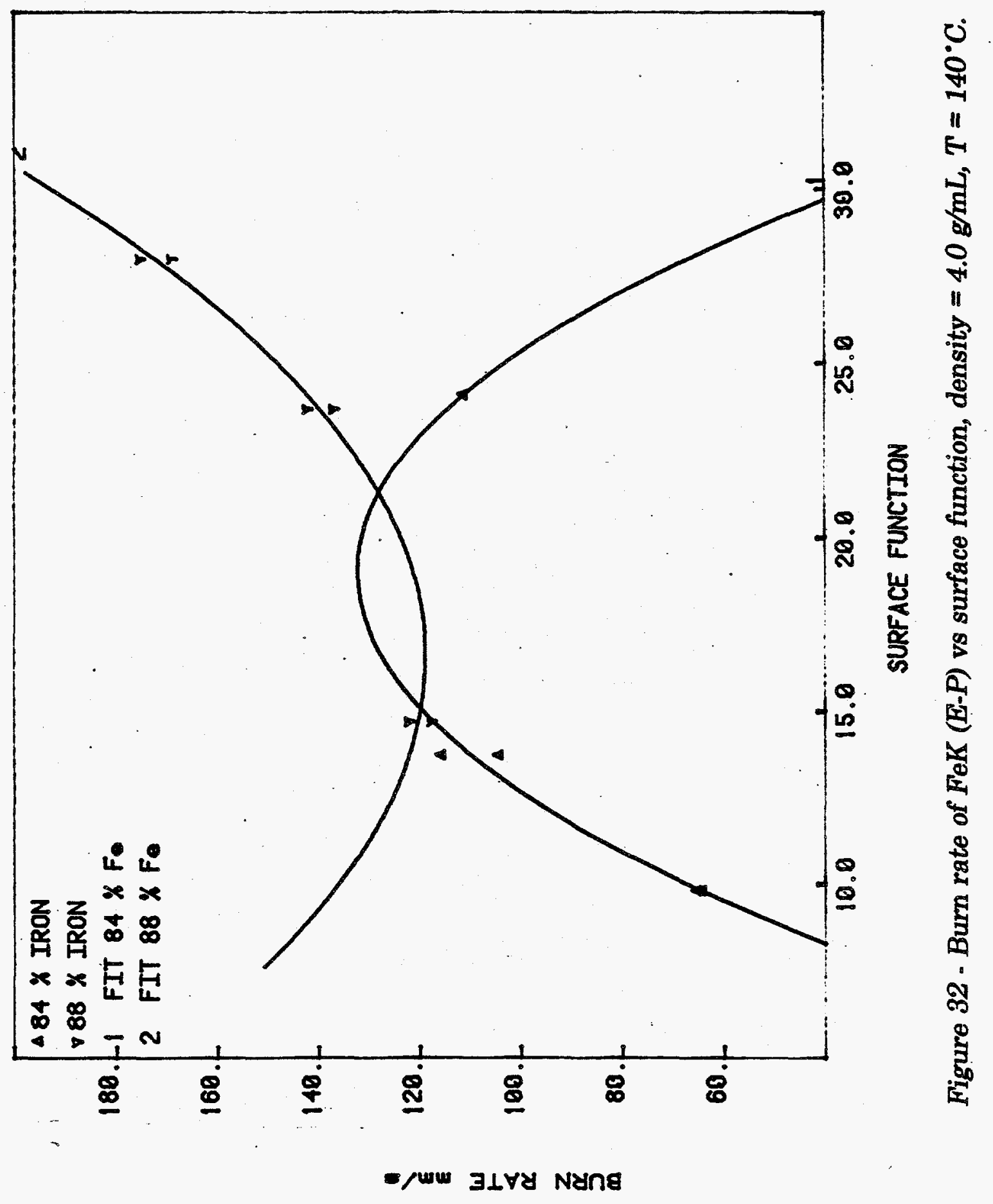




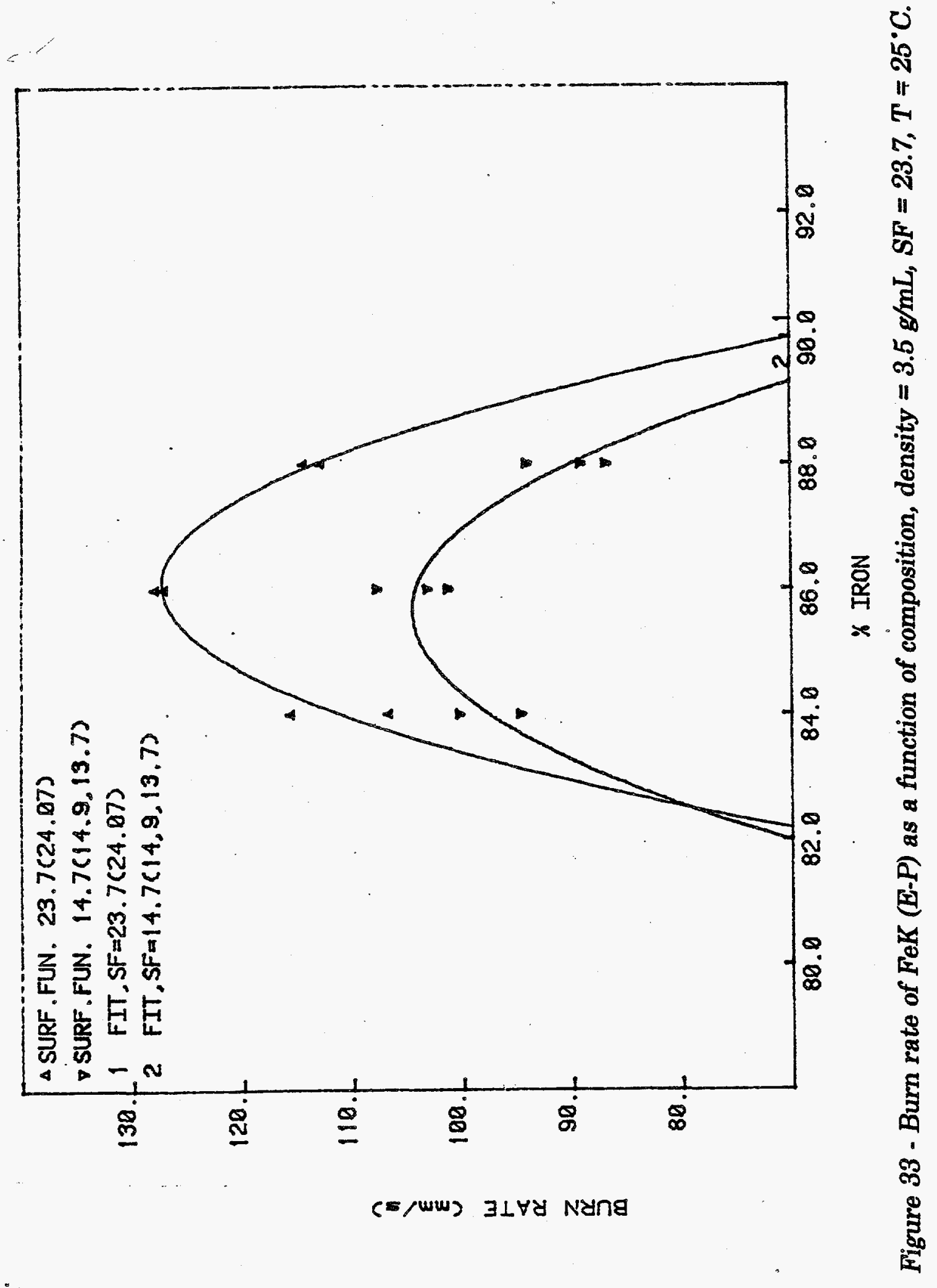




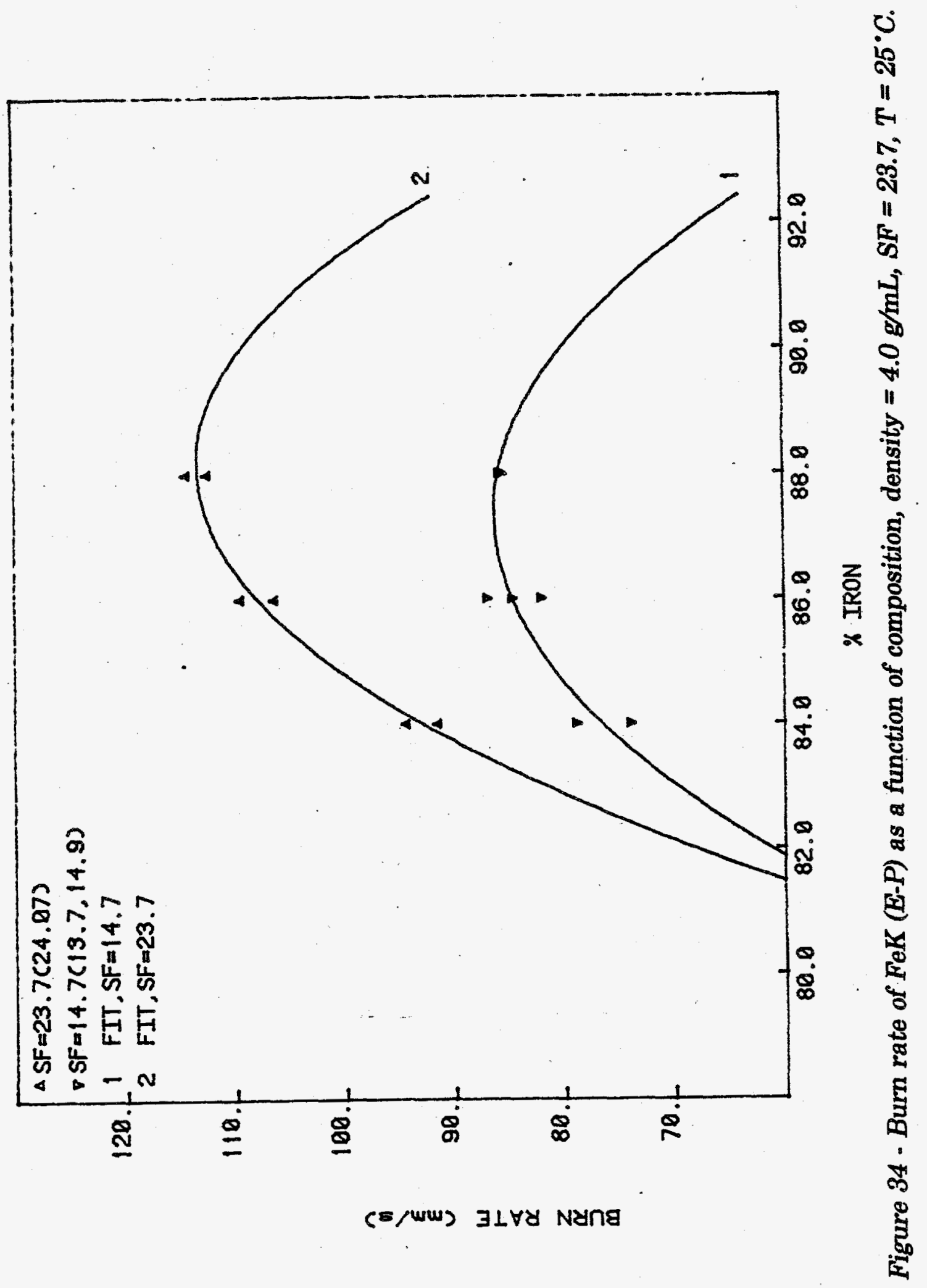




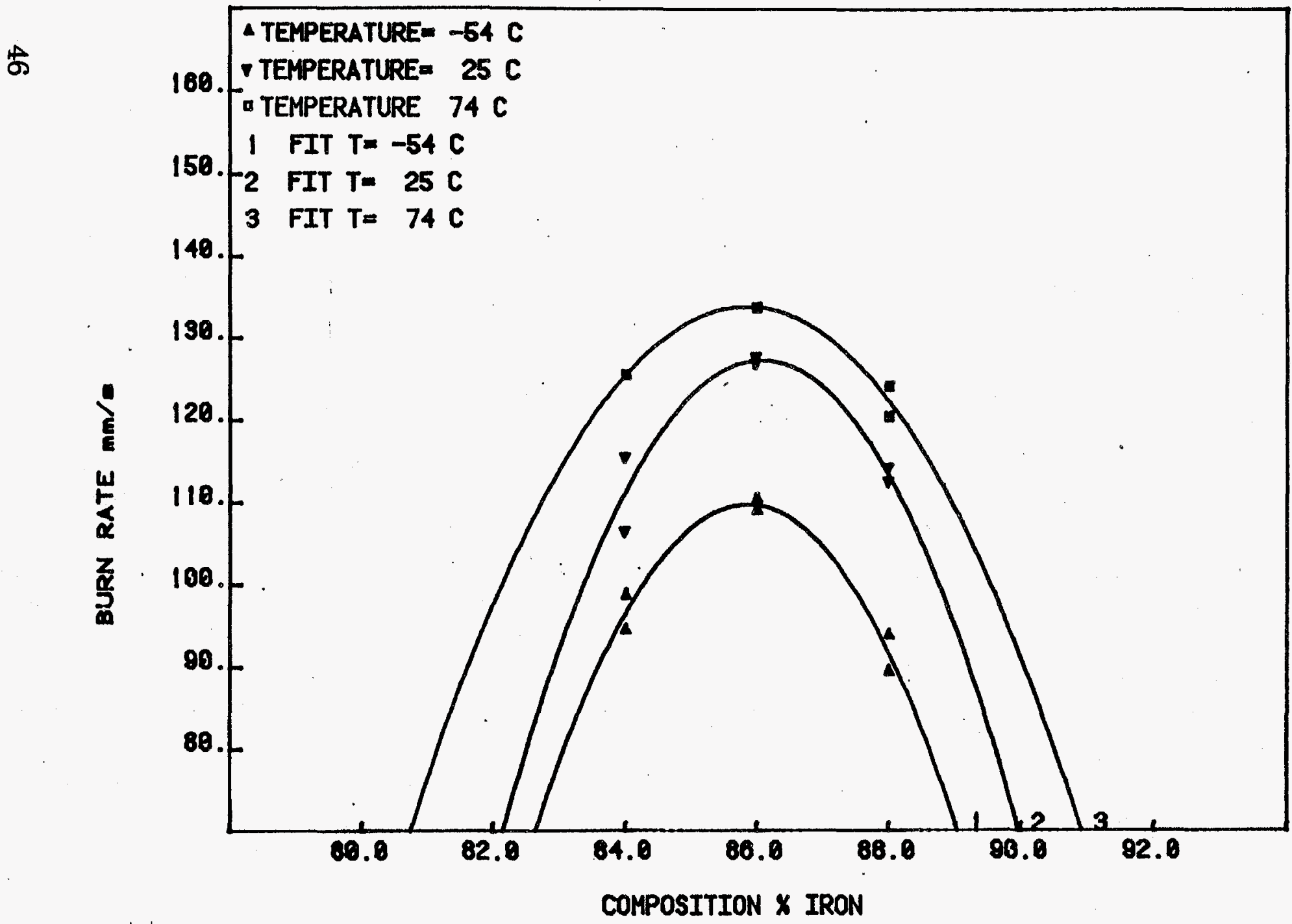

Figure 35 - Burn rate of FeK (E-P) as a function of composition, density $=3.5 \mathrm{~g} / \mathrm{mL}, S F=24$. 


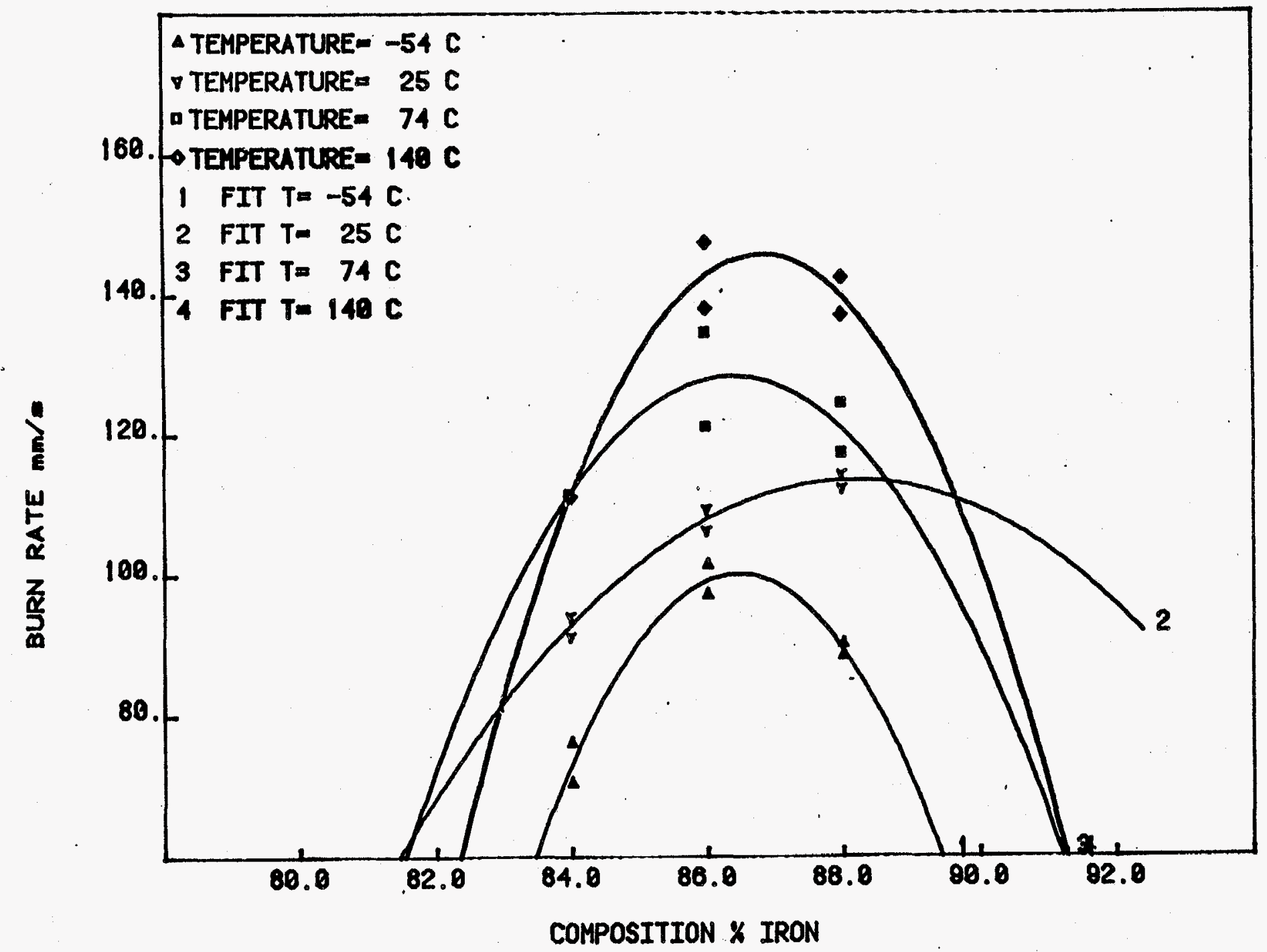

Figure 36 - Burn rate of $F e K(E-P)$ as a function of composition, density $=4.0 \mathrm{~g} / \mathrm{mL}, S F=24$. 
Table 3 shows the complete set of E-P burn rate data along with the computed burn rates from the twelve-parameter, empirical fitting equation. The form of the equation for burn rate, $R$, is

$$
\begin{gathered}
\mathrm{R}=\mathrm{B}(1)+\mathrm{B}(2)(\% \mathrm{Fe})+\mathrm{B}(3)(\% \mathrm{Fe})^{2}+\mathrm{B}(4)(\mathrm{SF})+\mathrm{B}(5)(\mathrm{SF})^{2}+\mathrm{B}(6)(\mathrm{SF})^{-1}+ \\
\mathrm{B}(7)(\mathrm{SF})^{-2}+\mathrm{B}(8)(\mathrm{g} / \mathrm{mL})+\mathrm{B}(9)(\mathrm{g} / \mathrm{mL})^{2}+\mathrm{B}(10)(\mathrm{g} / \mathrm{mL})^{-5}+\mathrm{B}(11)(\mathrm{T})+\mathrm{B}(12)(\mathrm{T})^{2}
\end{gathered}
$$

and the parametric constants for the fit are

$$
\begin{aligned}
& B(1)=-3.52038815 \\
& B(2)=85.5637589 \\
& B(3)=-.510404738 \\
& B(4)=-139.985498 \\
& B(5)=2.06127969 \\
& B(6)=-35929.1090 \\
& B(7)=127398.001 \\
& B(8)=69.4146518 \\
& B(9)=-13.8866183 \\
& B(10)=-168.842333 \\
& B(11)=0.169054658 \\
& B(12)=0.000344881956
\end{aligned}
$$

The standard deviation for the fit is $10.7 \mathrm{~mm} / \mathrm{s}$.

Typical plots showing the fit are contained in Figures 37 through 39, where the curves are computed from the twelve-parameter equation and the measured burn rate data are represented by discrete points. It is obvious from Figure 39 that the equation should not be used outside the range of the data. Even slightly lower values of surface function than the lowest observed values produce exaggerated errors using this equation. The curves in Figures 37 and 38, computed from the equation, are much shallower than might be expected from the data. Also, when they are compared to the plots in Figures 33 through 36 , the indications are again that computed values outside the data range will be in error beyond that indicated by the computed standard deviation from the fit. 


\begin{tabular}{|c|c|c|c|c|c|c|c|}
\hline \multicolumn{8}{|c|}{$\begin{array}{l}\text { Table } 3 \text { - BURN RATE OF FeK (E-P) AS A FUNCTION OF COMPOSITION, } \\
\text { SURFACE FUNCTION, DENSITY, AND TEMPARATURE }\end{array}$} \\
\hline \multicolumn{8}{|c|}{ POLYMOMIAL COEFFICIENTS } \\
\hline \multicolumn{8}{|c|}{$B(1)=-3.52038815 E+00$} \\
\hline \multicolumn{8}{|c|}{$B(\quad 2)=8.55637589 E+01$} \\
\hline \multicolumn{8}{|c|}{$B(\quad 3)=-5.10404738 E-01$} \\
\hline \multicolumn{8}{|c|}{ B( $\quad 4)=-1.39985498 E+02$} \\
\hline \multicolumn{8}{|c|}{$B(5)=2.06127969 E+00$} \\
\hline \multicolumn{8}{|c|}{$B(6)=-3.59291090 E+04$} \\
\hline \multicolumn{8}{|c|}{$8(\quad 7)=1.27398001 E+05$} \\
\hline \multicolumn{8}{|c|}{$B(8)=6.94146518 E+01$} \\
\hline \multicolumn{8}{|c|}{ B( 9$)=-1.38866183 E+01$} \\
\hline \multicolumn{8}{|c|}{$B(10)=-1.68842333 E+02$} \\
\hline \multicolumn{8}{|c|}{$B(11)=1.69054658 \mathrm{E}-01}$. \\
\hline \multicolumn{8}{|c|}{$B(12)=3.44881956 E-04$} \\
\hline$\#$ & * IRON & SURFACE FUNCTION & DENSITY $\mathrm{g} / \mathrm{ml}$ & TEMPERATURE $C$ & BURN RATE $\mathrm{mm} / \mathrm{s}$ & CALC & DELTA \\
\hline 1 & $8.60000000 E+01$ & $2.37000000 E+01$ & $3.00000000 E+00$ & $2.70000000 E+01$ & $1.22200000 E+02$ & $1.21568227 E+02$ & $6.31773455 E-01$ \\
\hline 2 & $8.60000000 E+01$ & $2.37000000 E+01$ & $3.00000000 E+00$ & $2.70000000 E+01$ & $1.24200000 \varepsilon+02$ & $1.21568227 \bar{Z}+02$ & $2.63177345 E+00$ \\
\hline 3 & $8.40000000 E+01$ & $2.40700000 E+01$ & $4.00000000 E+00$ & $7.40000000 E+01$ & $1.11500000 E+02$ & $1.19851847 E+02$ & $-8.35184715 E+00$ \\
\hline 4 & $8.60000000 E+01$ & $2.37000000 E+01$ & $3.00000000 E+00$ & $1.50000000 E+02$ & $1.45200000 E+02$ & $1.49870375 E+02$ & $-4.67037457 E+00$ \\
\hline 5 & $8.60000000 E+01$ & $2.37000000 \mathrm{E}+01$ & $3.00000000 E+00$ & $1.50000000 E+02$ & $1.55500000 E+02$ & $1.49870375 E+02$ & $5.62962543 E+00$ \\
\hline 6 & $8.60000000 E+01$ & $2.37000000 E+01$ & $3.00000000 E+00$ & $-5.40000000 E+01$ & $1.18400000 E+02$ & $1.08629056 E+02$ & $9.77094393 E+00$ \\
\hline 7 & $8.60000000 E+01$ & $2.37000000 E+01$ & $3.00000000 E+00$ & $-5.40000000 E+01$ & $9.89900000 E+01$ & $1.08629056 E+02$ & $-9.63905607 E+00$ \\
\hline 8 & $8.60000000 E+01$ & $2.37000000 E+01$ & $3.00000000 E+00$ & $-5.40000000 E+01$ & $1.07000000 E+02$ & $1.08629056 E+02$ & $-1.62905607 E+00$ \\
\hline 9 & $8.60000000 E+01$ & $2.37000000 E+01$ & $3.00000000 E+00$ & $-5.40000000 E+01$ & $1.04000000 E+02$ & $1.08629056 E+02$ & $-4.62905607 E+00$ \\
\hline 10 & $8.60000000 E+01$ & $2.37000000 E+01$ & $3.00000000 E+00$ & $-5.40000000 E+01$ & $1.05000000 E+02$ & $1.08629056 E+02$ & $-3.62905607 E+00$ \\
\hline 11 & $8.60000000 E+01$ & $2.37000000 E+01$ & $3.00000000 E+00$ & $7.40000000 E+01$ & $1.86300000 E+02$ & $1.31150950 E+02$ & $5.51490499 E+01$ \\
\hline 12 & $8.60000000 E+01$ & $2.37000000 E+01$ & $3.00000000 E+00$ & $7.40000000 E+01$ & $1.55500000 E+02$ & $1.31150950 E+02$ & $2.43490499 E+01$ \\
\hline 13 & $8.60000000 E+01$ & $2.37000000 E+01$ & $3.00000000 E+00$ & $7.40000000 E+01$ & $1.34500000 E+02$ & $1.31150950 E+02$ & $3.34904988 E+00$ \\
\hline 14 & $8.60000000 E+01$ & $2.37000000 E+01$ & $3.00000000 E+00$ & $7.40000000 E+01$ & $1.29200000 E+02$ & $1.31150950 E+02$ & $-1.95095012 E+00$ \\
\hline 15 & $8.60000000 E+01$ & $2.37000000 E+01$ & $3.50000000 E+00$ & $2.60000000 E+01$ & $1.27700000 E+02$ & $1.18187853 E+02$ & $9.51214738 E+00$ \\
\hline 16 & $8.60000000 E+01$ & $2.37000000 E+01$ & $3.50000000 E+00$ & $2.60000000 E+01$ & $1.26900000 E+02$ & $1.18187853 E+02$ & $8.71214738 E+00$ \\
\hline 17 & $8.60000000 E+01$ & $2.37000000 E+01$ & $3.50000000 E+00$ & $2.60000000 E+01$ & $1.26900000 E+02$ & $1.18187853 E+02$ & $8.71214738 E+00$ \\
\hline
\end{tabular}


Table 3 - BURN RATE OF FeK (E-P) AS A FUNCTION OF COMPOSITION, SURFACE FUNCTION, DENSITY, AND TEMPERATURE (continued)

\section{$3.50000000 E+00$}

$3.50000000 E+00$

$3.50000000 E+00$

$3.50000000 E+00$

$3.50000000 E+00$

$3.50000000 E+00$

$3.50000000 \mathrm{E}+00$

$3.50000000 E+00$

$4.00000000 \mathrm{E}+00$

$4.00000000 E+00$

$4.00000000 E+00$

$4.00000000 E+00$

$4.00000000 E+00$

4. $00000000 E+00$

$4.00000000 E+00$

4. $00000000 E+00$

$3.50000000 E+00$

$3.50000000 \mathrm{E}+00$

$3.50000000 E+00$

$3.50000000 E+00$

$3.50000000 \mathrm{E}+00$

$3.50000000 E+00$

$3.50000000 E+00$

$3.50000000 E+00$

$4.00000000 E+00$

4.00000000E+00

$4.00000000 E+00$

$4.00000000 E+00$

$4.00000000 E+00$

4.00000000E+00

$4.00000000 E+00$

4. $00000000 E+00$

$3.50000000 E+00$

$3.50000000 E+00$

$3.50000000 E+00$

$3.50000000 E+00$
$1.40000000 E+02$

$1.40000000 E+02$

$-5.40000000 E+01$

$-5.40000000 E+01$

7.40000000E+01

$1.40000000 \mathrm{E}+02$

$-1.20000000 E+02$

$-1.20000000 E+02$

2.40000000E+0.1

2.40000000E+01

1.35000000E+02

1.35000000E+02

$-5.40000000 E+01$

$-5.40000000 E+01$

$7.40000000 E+01$

$7.40000000 E+01$

$1.40000000 \mathrm{E}+02$

$1.40000000 E+02$

$-5,40000000 E+01$

$-5.40000000 E+01$

2.50000000E+01

2.50000000E+01

7.40000000E+01

7. $40000000 E+01$

$1.40000000 \mathrm{E}+02$

$1.40000000 E+02$

2.50000000E+01

2.50000000E+01

$-5.40000000 E+01$

$-5.40000000 E+01$

7.40000000E +01

$7.40000000 E+01$

2.70000000E+01

2.70000000E+01

$1.40000000 E+02$

1.40000000E+02
$1.59400000 E+02$

$1.57400000 E+02$

$1.08900000 \mathrm{E}+02$

1. $10300000 E+02$

$1.33600000 E+02$

$1.40000000 E+02$

$9.92700000 E+01$

$1.02400000 \mathrm{E}+02$

$1.06300000 E+02$

$1.09400000 E+02$

$1.38000000 E+02$

$1.47400000 E+02$

$9.71300000 E+0.1$

$1.01300000 E+02$

$1.21000000 E+02$

$1.34500000 E+02$

$1.63000000 E+02$

$1.64200000 E+02$

$1.06800000 \mathrm{E}+02$

$1.08300000 E+02$

$1.29800000 E+02$

$1.24800000 E+02$

$1.41070000 E+02$

$1.44800000 E+02$

$1.69300000 E+02$

$1.75500000 E+02$

$1.27000000 E+02$

1. $26000000 \mathrm{E}+02$

$1.07900000 E+02$

$1.08500000 E+02$

$1.43300000 \mathrm{E}+02$

$1.48100000 E+02$

$5.47900000 E+01$

5.40400000E+01

8.06500000E+01

$7.66300000 \mathrm{E}+01$
$1.439866630 E+02$

$1.43986630 \mathrm{E}+02$

$1.05436016 E+02$

$1.05436016 \mathrm{E}+02$

$1.27957910 E+02$

$1.43986630 E+02$

$9.82390325 E+01$

$9.82390325 E+01$

$1.06276619 E+02$

$1.06276619 E+02$

$1.31128508 E+02$

$1.31128508 E+02$

$9.38973794 \mathrm{E}+01$

$9.38973794 E+01$

$1.16419273 E+02$

$1.16419273 E+02$

$1.62297948 E+02$

$1.62297948 E+02$

$1.23747334 E+02$

$1.23747334 E+02$

$1.36312527 E+02$

$1.36312527 \mathrm{E}+02$

$1.46269228 E+02$

$1.46269228 E+02$

$1.50759312 E+02$

$1.50759312 E+02$

$1.24773891 \mathrm{E}+02$

$1.24773891 E+02$

$1.12208697 \mathrm{E}+02$

$1.12208697 \mathrm{E}+02$

1.34730592E+02

$1.34730592 E+02$

5.62072156E+01

$5.62072156 E+01$

$8.18186593 E+01$

8.18186593E+01
$1.54133702 E+01$

$1.34133702 E+01$

$3.46398445 E+00$

$4.86398445 E+00$

$5.64209040 E+00$

$-3.98662979 E+00$

$1.03096751 E+00$

4. $16096751 E+00$

2.33810786E-02

$3.12338108 E+00$

$6.87149238 E+00$

$1.62714924 E+01$

$3.23262064 \mathrm{E}+00$

$7.40262064 E+00$

$4.58072659 E+00$

$1.80807266 E+01$

7.02052119E-01

$1.90205212 E+00$

$-1.69473336 E+01$

$-1.54473336 E+01$

$-6.51252708 E+00$

$-1.15125271 E+01$

-5.19922770E+00

$-1.46922770 E+00$

$1.85406883 \mathrm{E}+01$

$2.47406883 E+01$

2.22610911E+00

$1.22610911 E+00$

$-4.30869746 E+00$

$-3.70869746 E+00$

8.56940849E+0D

$1.33694085 E+01$

$-1.41721557 E+00$

$-2.16721557 E+00$

$-1.16865934 E+00$

$-5.18865934 E+00$ 
Table 3 - BURN RATE OF FeK (E-P) AS A FUNCTION OF COMPOSITION, SURFACE FUNCTION, DENSITY, AND TEMPERATURE (continued)

\begin{tabular}{|c|c|c|c|c|c|c|c|}
\hline 54 & $8.40000000 E+01$ & $9.80000000 E+00$ & $3.50000000 E+00$ & $-5.40000000 E+01$ & $5.06100000 E+01$ & $4.32680451 E+01$ & $7.34195490 \mathrm{E}+00$ \\
\hline 55 & $8.40000000 E+01$ & $9.80000000 E+00$ & $3.50000000 E+00$ & $-5.40000000 \mathrm{E}+01$ & $4.86100000 E+01$ & $4.32680451 E+01$ & $5.34195490 \mathrm{E}+00$ \\
\hline 56 & $8.40000000 E+01$ & $9.80000000 E+00$ & $3.50000000 E+00$ & $-5.40000000 E+01$ & $5.04000000 E+01$ & $4.32680451 E+01$ & $7.13195490 E+00$ \\
\hline 57 & $8.40000000 E+01$ & $9.80000000 E+00$ & $3.50000000 \mathrm{E}+00$ & $7.40000000 E+01$ & $6.61900000 E+01$ & $6.57899391 E+01$ & $4.00060850 E-01$ \\
\hline 58 & $8.40000000 E+01$ & $9.80000000 E+00$ & $3.50000000 E+00$ & $7.40000000 E+01$ & $7.05000000 E+01$ & $6.57899391 E+01$ & $4.71006085 \mathrm{E}+00$ \\
\hline 59 & $8.40000000 E+01$ & $9.80000000 E+00$ & $4.00000000 E+00$ & $2.80000000 E+01$ & $4.52700000 E+01$ & $4.48566026 \mathrm{E}+01$ & $4.13397449 E-01$ \\
\hline 60 & $8.40000000 E+01$ & $9.80000000 E+00$ & $4.00000000 E+00$ & $2.80000000 E+01$ & $4.87800000 E+01$ & $4.48566026 E+01$ & $3.92339745 E+00$ \\
\hline 61 & $8.40000000 E+01$ & $9.80000000 E+00$ & $4.00000000 E+00$ & $1.40000000 E+02$ & $6.41600000 E+01$ & $7.02800232 E+01$ & $-6.12002315 E+00$ \\
\hline 62 & $8.40000000 E+01$ & $9.80000000 E+00$ & $4.00000000 E+00$ & $1.40000000 E+02$ & $6.49700000 E+01$ & $7.02800232 E+01$ & $-5.31002315 E+00$ \\
\hline 63 & $8.40000000 E+01$ & $9.80000000 E+00$ & $4.00000000 E+00$ & $-5.40000000 E+01$ & $4.26500000 E+01$ & 3.17294089E+01 & $1.09205911 E+01$ \\
\hline 64 & $8.40000000 E+01$ & $9.80000000 E+00$ & $4.00000000 E+00$ & $-5.40000000 E+01$ & $4.18600000 E+01$ & 3.17294089E+01 & $1.01305911 E+01$ \\
\hline 65 & $8.40000000 E+01$ & $9.80000000 E+00$ & $4.00000000 E+00$ & $7.40000000 \mathrm{E}+01$ & $5.45200000 E+01$ & $5.42513030 E+01$ & $2.68697035 E-01$ \\
\hline 66 & $8.40000000 E+01$ & $9.80000000 E+00$ & $4.00000000 E+00$ & $7.40000000 E+01$ & $5.45100000 E+01$ & $5: 42513030 \mathrm{E}+01$ & $2.58697035 E-01$ \\
\hline 67 & B. $60000000 E+01$ & $1.10000000 E+01$ & $3.50000000 E+00$ & $2.50000000 E+01$ & $5.66800000 E+01$ & $6.32093077 \mathrm{E}+01$ & $-6.52930765 E+00$ \\
\hline 68 & $8.60000000 E+01$ & $1.10000000 E+01$ & $3.50000000 E+00$ & $2.50000000 E+01$ & $5.96100000 E+01$ & $6.32093077 \mathrm{E}+01$ & $-3.59930765 E+00$ \\
\hline 69 & $8.60000000 E+01$ & $1.10000000 E+01$ & $3.50000000 E+00$ & $1.40000000 E+02$ & $7.38100000 E+01$ & $8.91947285 E+01$ & $-1.53847285 E+01$ \\
\hline 70 & $8.60000000 E+01$ & $1.10000000 E+01$ & $3.50000000 E+00$ & $1.40000000 E+02$ & $7.31600000 E+01$ & $8.91947285 E+01$ & $-1.60347285 E+01$ \\
\hline 71 & $8.60000000 E+01$ & $1.10000000 E+01$ & $3.50000000 E+00$ & $-5.40000000 E+01$ & $5.01100000 E+01$ & $5.06441142 E+01$ & $-5.34114219 E-01$ \\
\hline 72 & $8.60000000 E+01$ & $1.10000000 E+01$ & $3.50000000 E+00$ & $-5.40000000 E+01$ & $5.11000000 E+01$ & $5.06441142 E+01$ & $4.55885781 E-01$ \\
\hline 73 & $8.60000000 E+01$ & $1.10000000 E+01$ & $3.50000000 E+00$ & $7.40000000 E+01$ & $6.35100000 E+01$ & $7.31660083 E+01$ & $-9.65600827 E+00$ \\
\hline 74 & $8.60000000 E+01$ & $1.10000000 \mathrm{E}+01$ & $3.50000000 E+00$ & $7.40000000 E+01$ & $6.28600000 E+01$ & $7.31660083 E+01$ & $-1.03060083 E+01$ \\
\hline 75 & $8.60000000 E+01$ & $1.10000000 E+01$ & $4.00000000 E+00$ & $2.50000000 E+01$ & $4.89500000 \mathrm{E}+01$ & $5.16706715 E+01$ & $-2.72067147 E+00$ \\
\hline 76 & $8.60000000 E+01$ & $1.10000000 E+01$ & $4.00000000 E+00$ & $2.50000000 E+01$ & $4.96300000 E+01$ & $5.16706715 E+01$ & $-2.04067147 E+00$ \\
\hline 77 & $8.60000000 E+01$ & $1.10000000 E+01$ & $4.00000000 E+00$ & $1.40000000 E+02$ & $6.83100000 E+01$ & $7.76560923 E+01$ & $-9.34609228 E+00$ \\
\hline 78 & $8.60000000 E+01$ & $1.10000000 E+01$ & $4.00000000 E+00$ & $1.40000000 E+02$ & $6.47600000 E+01$ & $7.76560923 E+01$ & $-1.28960923 E+01$ \\
\hline 79 & $8.60000000 E+01$ & $1.10000000 E+01$ & $4.00000000 E+00$ & $-5.40000000 E+01$ & $4.52300000 E+01$ & $3.91054780 E+01$ & $6.12452197 E+00$ \\
\hline 80 & $8.60000000 E+01$ & $1.10000000 E+01$ & $4.00000000 E+00$ & $-5.40000000 E+01$ & $4.20200000 E+01$ & $3.91054780 E+0.1$ & $2.91452197 E+00$ \\
\hline 81 & $8.60000000 E+01$ & $1.10000000 E+01$ & $4.00000000 E+00$ & $7.40000000 E+01$ & $5.82400000 E+01$ & $6.16273721 E+01$ & $-3.38737209 E+00$ \\
\hline 82 & $8.60000000 E+01$ & $1.10000000 E+01$ & $4.00000000 E+00$ & $7.40000000 E+01$ & $5.77300000 E+01$ & $6.16273721 E+01$ & $-3.89737209 E+00$ \\
\hline 83 & $8.80000000 E+01$ & $1.47000000 E+01$ & $3.00000000 E+00$ & $7.40000000 E+01$ & $9.78700000 \mathrm{E}+01$ & $1.06735891 E+02$ & $-8.86589088 E+00$ \\
\hline 84 & $8.80000000 E+01$ & $1.47000000 E+01$ & $3.00000000 E+00$ & $7.40000000 E+01$ & $1.00400000 E+02$ & $1.06735891 E+02$ & $-6.33589088 E+00$ \\
\hline 85 & $8.80000000 E+01$ & $1.47000000 E+01$ & $3.00000000 E+00$ & $7.40000000 E+01$ & $9.64000000 E+01$ & $1.06735891 E+02$ & $-1.03358909 E+01$ \\
\hline 86 & $8.80000000 E+01$ & $1.47000000 E+01$ & $3.00000000 E+00$ & $7.40000000 E+01$ & $1.02300000 E+02$ & $1.06735891 E+02$ & $-4.43589088 E+00$ \\
\hline 87 & $8.80000000 E+01$ & $1.47000000 E+01$ & $3.00000000 E+00$ & $7.40000000 E+01$ & $1.04080000 E+02$ & $1.06735891 E+02$ & $-2.65589088 E+00$ \\
\hline 88 & $8.80000000 E+01$ & $1.47000000 E+01$ & $3.00000000 E+00$ & $7.40000000 E+01$ & $1.02200000 E+02$ & $1.06735891 E+02$ & $-4.53589088 E+00$ \\
\hline 89 & $8.80000000 E+01$ & $1.47000000 E+01$ & $3.00000000 E+00$ & $2.50000000 E+01$ & 8.76000000E+01 & $9.67791903 E+01$ & $-9.17919026 E+00$ \\
\hline
\end{tabular}


Table 3 - BURN RATE OF FeK (E-P) AS A FUNCTION OF COMPOSITION, SURFACE FUNCTION, DENSITY, AND TEMPERATURE (continued)

$1.47000000 E+01$

$1.47000000 E+01$

$1.47000000 E+01$

$1.47000000 E+01$

$1.47000000 E+01$

$1.47000000 E+01$

$1.47000000 E+01$

$1.47000000 E+01$

$1.47000000 E+01$

$1.47000000 E+01$

$1.47000000 E+01$

$1.47000000 E+01$

$1.47000000 E+01$

$1.47000000 E+01$

$1.47000000 E+01$

1.47000000E+01

$1.47000000 \mathrm{E}+01$

$1.47000000 E+01$

$1.47000000 \mathrm{E}+01$

$1.47000000 E+01$

$1.47000000 E+01$

$1.47000000 \mathrm{E}+01$

$1.47000000 E+01$

1.47000000E+01

1.37000000E+01

$1.37000000 E+01$

$1.37000000 E+01$

$1.37000000 E+01$

$1.37000000 E+01$

1.37000000E+01

$1.37000000 E+01$

1.37000000E+01

1.37000000E+01

1.37000000E+01

1.37000000E+01

1.37000000E +01
$3.00000000 E+00$

$3.00000000 E+00$

$3.00000000 E+00$

$3.00000000 E+00$

$3.00000000 E+00$

$3.00000000 E+00$

$3.00000000 E+00$

$3.50000000 E+00$

$3.50000000 E+00$

$3.50000000 E+00$

$3.50000000 \mathrm{E}+00$

$3.50000000 E+00$

$3.50000000 E+00$

3.50000000E +00

$3.50000000 E+00$

$3.50000000 E+00$

$4.00000000 E+00$

4.00000000E+00

$4.00000000 \mathrm{E}+00$

4. $00000000 E+00$

$4.00000000 E+00$

$4.00000000 E+00$

$4.00000000 E+00$

4.00000000E+00

$4.00000000 E+00$

$4.00000000 E+00$

$4.00000000 E+00$

$4.00000000 \mathrm{E}+00$

$4.00000000 E+00$

$4.00000000 E+00$

$4.00000000 E+00$

$4.00000000 E+00$

$5.00000000 \mathrm{E}+00$

5.00000000E+00

5. $00000000 E+00$

5. $00000000 E+00$
2.50000000E+01 $2.50000000 E+09$

$2.50000000 E+01$

2.50000000E+0

$-5.40000000 E+01$

$-5.40000000 E+01$

$1.46000000 E+02$

2.50000000E+01

$2.50000000 E+01$

2.50000000E+01

$7.40000000 E+09$

$7.40000000 E+01$

$-5.40000000 E+01$

$-5.40000000 E+01$

$1.25000000 E+02$

$1.25000000 E+02$

$1.58000000 \mathrm{E}+02$

$1.58000000 E+02$

$-5.40000000 E+01$

$-5.40000000 E+01$

$7.40000000 E+01$

$7.40000000 E+01$

$2.50000000 E+01$

$2.42000000 E+01$

2.50000000E+01

$2.50000000 E+01$

$1.40000000 E+02$

$9.40000000 E+02$

$-5.40000000 E+01$

$-5.40000000 E+01$

$7.40000000 E+01$

7.40000000E+01

2.70000000E+01

2.70000000E+01

1. $40000000 E+02$

$1.40000000 E+02$
8.78000000E+01

8.77000000E+01

8.77000000E+01

8.69300000E+01

$7.44100000 E+01$

$7.40400000 E+01$

$1.31000000 E+02$

8.69300000E+01

$8.91800000 E+01$

$9.41000000 \mathrm{E}+01$

$1.03750000 E+02$

$1.05900000 E+02$

$7.75900000 E+01$

$7.82800000 \mathrm{E}+01$

1.15000000E+02

$1.21800000 \mathrm{E}+02$

$1.17900000 \mathrm{E}+02$

$1.22200000 E+02$

7.17000000E+01

7.25700000E+01

$9.44600000 E+01$

$9.82200000 E+01$

8. $55500000 E+01$

8.59300000E+01

$7.40200000 E+01$

$7.89300000 E+01$

1.04200000E+02

1. $15600000 E+02$

7.21400000E+01

$6.86700000 E+01$

9.07900000E+01

$9.93200000 E+01$

4.12600000E+01

$4.56300000 E+01$

$6.96200000 E+01$

$6.51000000 E+01$
$9.67791903 E+01$ $9.67791903 E+01$ $9.67791903 E+01$

$9.67791903 E+01$

$8.42139968 E+01$

$8.42139968 E+01$

$1.24370756 E+02$

9.35861497E+01

9.35861497E+01

9.35861497E+01

$1.03542850 E+02$

$1.03542850 E+02$

$8.10209563 E+01$

8.10209563E+01

$1.15664845 E+02$

1.15664845E+02

$1.12925865 E+02$

$1.12925865 E+02$

$6.94823201 E+01$

$6.94823201 E+01$

$9.20042142 E+01$

$9.20042142 E+01$

8. 20475136E+01

8.18986953E+01

B.31987434E+01

8.31987434E+01

$1.09184164 E+02$

$1.09184164 E+02$

$7.06335500 E+01$

$7.06335500 \mathrm{E}+01$

$9.31554440 E+01$

$9.31554440 E+01$

$3.69203868 \mathrm{E}+01$

$3.69203868 E+01$

$6.25318306 \mathrm{E}+01$

$6.25318306 E+01$
$-8.97919026 E+00$

$-9.07919026 E+00$

$-9.07919026 E+00$

$-9.84 \div 19026 E+00$

$-9.80399683 E+00$

$-1.01739968 E+01$

$6.62924355 E+00$

$-6.65614974 E+00$

$-4.40614974 E+00$

5.13850264E-01

2.07149644E-01

$2.35714964 \mathrm{E}+00$

$-3.43095630 E+00$

$-2.74095630 E+00$

$-6.64844894 E-01$

$6.13515511 E+00$

$4.97413498 \mathrm{E}+00$

$9.27413498 E+00$

$2.21767988 E+00$

$3.08767988 E+00$

$2.45578583 E+00$

$6.21578583 E+00$

$3.50248645 E+00$

4.03130473E+00

$-9.17874343 E+00$

$-4.26874343 E+00$

$-4.98416423 E+00$

$6.415835 T 7 E+00$

$1.50645001 E+00$

$-1.96354999 E+00$

$-2.36544405 E+00$

$6.16455595 E+00$

4.33961315E+00

8.70961315E+00

$7.08816939 \mathrm{E}+00$

$2.56816939 E+00$ 


\section{Table 3 - BURN RATE OF FeK (E-P) AS A FUNCTION OF COMPOSHTION,}

SURFACE FUNCTION, DENSITY, AND TEMPERATURE (continued)

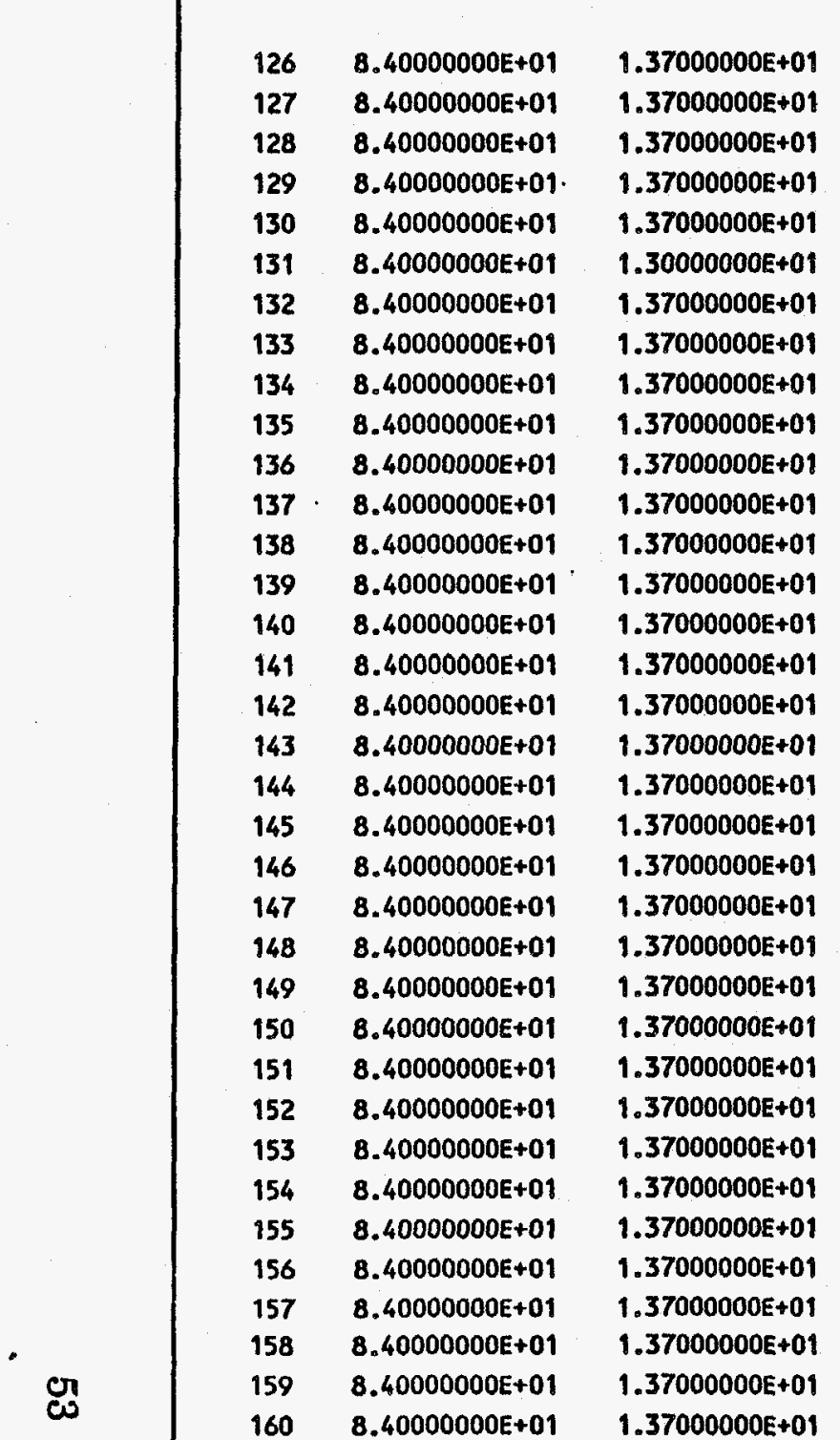

$5.00000000 E+00 \quad-5.40000000 E+01$ $5.00000000 E+00$

$5.00000000 E+00$

$5.00000000 E+00$

$3.30000000 E+00$

$3.30000000 E+00$

$3.30000000 \mathrm{E}+00$

$3.30000000 E+00$

$3.30000000 E+00$

$3.30000000 E+00$

3. 30000000E +00

3. $30000000 E+00$

3. $50000000 \mathrm{E}+00$

$3.50000000 \mathrm{E}+00$

$3.50000000 E+00$

$3.50000000 E+00$

3.50000000E +00

$3.50000000 E+00$

$3.50000000 \mathrm{E}+00$

$3.50000000 \mathrm{E}+00$

2.50000000E +00

$2.50000000 E+00$

$2.50000000 \mathrm{E}+00$

$2.50000000 E+00$

$2.50000000 \mathrm{E}+00$

$2.50000000 E+00$

$2.50000000 E+00$

$2.50000000 E+00$

$2.50000000 E+00$

2.50000000E +00

$3.00000000 E+00$

$3.00000000 E+00$

$3.00000000 E+00$

$3.00000000 \mathrm{E}+00$

$3.00000000 E+00$
$-5.40000000 E+01$

7.40000000E+01

$7.40000000 \mathrm{E}+01$

$2.63000000 E+01$

$2.63000000 E+01$

$1.40000000 E+02$

$1.40000000 E+02$

$-5.40000000 E+01$

$-5.40000000 E+01$

$7.40000000 \mathrm{E}+01$

$7.40000000 E+01$

2.59000000E+01

$2.59000000 E+01$

$1.40000000 E+02$

$1.40000000 E+02$

$-5.40000000 E+01$

$-5.40000000 E+01$

$7.40000000 \mathrm{E}+01$

$7.40000000 E+01$

$2.33000000 E+01$

2.33000000E+01

$1.40000000 E+02$

$1.40000000 E+02$

$-5.40000000 E+01$

$-5.40000000 E+01$

$7.40000000 E+01$

$7.40000000 E+01$

7.40000000E+01

$7.40000000 E+01$

$2.50000000 E+01$

$2.50000000 E+01$

$1.40000000 E+02$

$1.40000000 \mathrm{E}+02$

$-5.40000000 E+01$
$3.83700000 E+01$

$3.86300000 E+01$

$5.92700000 E+01$

5.77400000E+01

$9.76300000 \mathrm{E}+01$

$1.05030000 E+02$

$1.31350000 E+02$

$1.28810000 \mathrm{E}+02$

$9.21900000 \mathrm{E}+01$

$9.50300000 \mathrm{E}+01$

1.12400000E+02

$1.16200000 E+02$

$9.46800000 E+01$

$1.00330000 E+02$

1.22220000E +02

$1.27160000 \mathrm{E}+02$

8.73400000E+01

$8.83700000 E+01$

$1.13400000 \mathrm{E}+02$

$1.11300000 E+02$

$9.65300000 E+01$

$1.04700000 E+02$

$5.40400000 E+01$

$5.51400000 \mathrm{E}+01$

$9.57700000 E+01$

$9.80700000 E+01$

$1.15900000 E+02$

$1.26300000 E+02$

$1.14600000 \mathrm{E}+02$

$1.26200000 E+02$

$9.99200000 E+01$

$1.04010000 E+02$

$1.27000000 E+02$

$1.30000000 \mathrm{E}+02$

9.57700000E+01
2.39812164E+01 2.39812164E+01 $4.65031104 E+01$ 4.65031104E+01

$9.72883993 E+01$ $9.06041544 E+01$ $1.23031049 E+02$ $1.23031049 E+02$ $8.44804346 E+01$ $8.44804346 E+01$ $1.07002329 E+02$ $1.07002329 E+02$ $9.49053278 E+0$ $9.49053278 E+0$ $1.20722800 \mathrm{E}+02$ 1.20722800E+02 8.21721862E+01 $8.21721862 E+01$ 1.04694080E+02 $1.04694080 E+02$ $9.17914824 E+01$

$9.17914824 E+01$

$1.18092614 E+02$

$1.18092614 E+02$

$7.95420001 E+01$

$7.95420001 E+01$

$1.02063894 E+02$

$1.02063894 E+02$

$1.02063894 E+02$

$1.02063894 E+02$

$9.79304201 E+01$

$9.79304201 E+01$

$1.23915841 E+02$

$1.23915841 \mathrm{E}+02$

$8.53652267 \mathrm{E}+01$
$1.43887836 E+01$

$1.46487836 E+01$

$1.276688896 \mathrm{E}+01$

$1.12368896 E+01$

3.41600682E-01

$1.44258456 E+01$

$8.31895111 \mathrm{E}+00$

5.77895111E+00

$7.70956535 E+00$

1.05495654E+01

$5.39767130 E+00$

9.19767130E+00

$-2.25327845 E-01$

$5.42467215 E+00$

$1.49719958 E+00$

$6.43719958 E+00$

$5.16781382 E+00$

6. $19781382 E+00$

8. $70591977 E+0 D$

$6.60591977 E+00$

4.73851763E+00

$1.29085176 E+01$

$-6.40526144 E+01$

$-6.29526144 E+01$

$1.62279999 E+01$

$1.85279999 E+01$

$1.38361058 E+01$

$2.42361058 E+01$

1. $25361058 E+01$

$2.41361058 E+01$

$1.98957986 \mathrm{E}+00$

$6.07957986 E+00$

$3.08415906 E+00$

6.08415906E+00

$1.04047733 E+01$ 
Table 3 - BURN RATE OF FeK (E-P) AS A FUNCTION OF COMPOSITION, SURFACE FUNCTION, DENSITY, AND TEMPERATURE (continued)

$1.37000000 E+01$ $1.37000000 E+01$

$1.37000000 \mathrm{E}+01$

$1.49000000 E+01$

$1.49000000 E+01$

$1.49000000 \mathrm{E}+01$

$1.49000000 E+01$

$1.49000000 \mathrm{E}+01$

$1.49000000 E+01$

$1.49000000 E+01$

$1.49000000 \mathrm{E}+01$

$1.49000000 \mathrm{E}+01$

$1.49000000 \mathrm{E}+01$

$1.49000000 E+0$

$1.49000000 E+01$

$1.49000000 \mathrm{E}+01$

$1.49000000 \mathrm{E}+01$

$1.49000000 E+01$

$1.49000000 \mathrm{E}+01$

$1.49000000 E+01$

$1.49000000 E+01$

$1.49000000 \mathrm{E}+01$

$1.49000000 E+01$

$1.49000000 E+01$

$1.49000000 \mathrm{E}+01$

$1.49000000 E+01$

$1.49000000 E+01$

$1.49000000 E+01$

$1.49000000 E+01$

$1.49000000 E+01$

$1.49000000 \mathrm{E}+01$

$1.49000000 E+01$

$1.49000000 \mathrm{E}+01$

$1.49000000 \mathrm{E}+01$

$1.49000000 E+01$
$3.00000000 E+00$

3.00000000E+00

3. $00000000 E+00$

2.50000000E+00

2.50000000E+00

2.50000000E+00

2.50000000E+00

3. $00000000 E+00$

2.50000000E+00

2.50000000E +00

$3.00000000 E+00$

3.00000000E+00

3. $00000000 E+00$

$3.00000000 E+00$

$3.00000000 E+00$

$3.30000000 E+00$

3.30000000E+00

$3.30000000 E+00$

$3.30000000 E+00$

$3.30000000 E+00$

$3.30000000 E+00$

$3.50000000 E+00$

$3.50000000 E+00$

$3.50000000 E+00$

$3.50000000 E+00$

$3.50000000 E+00$

$3.50000000 E+00$

4. $00000000 E+00$

$4.00000000 \mathrm{E}+00$

$4.00000000 \varepsilon+00$

4. $00000000 E+00$

$4.00000000 E+00$

4. $00000000 E+00$

$5.00000000 E+00$

5. $00000000 E+00$
$-5.40000000 E+01$

$7.40000000 E+01$

$7.40000000 E+01$

$7.40000000 E+01$

$7.40000000 E+01$

$7.40000000 E+01$

7.40000000E+01

$7.40000000 E+01$

$7.40000000 E+01$

$7.40000000 E+01$

$7.40000000 E+01$

$7.40000000 E+01$

$7.40000000 E+01$

$7.40000000 E+01$

$7.40000000 E+01$

$7.40000000 E+01$

$7.40000000 \mathrm{E}+01$

$7.40000000 E+01$

$7.40000000 E+01$

$7.40000000 E+01$

$7.40000000 E+01$

$7.40000000 E+01$

$7.40000000 E+01$

$7.40000000 E+01$

$7.40000000 E+01$

$7.40000000 E+01$

$7.40000000 E+01$

$7.40000000 E+01$

$7.40000000 E+01$

$7.40000000 E+01$

$7.40000000 E+01$

$7.40000000 E+01$

$7.40000000 E+01$

$7.40000000 E+01$

$7.40000000 E+01$
9. $80700000 E+01$

$1.14500000 E+02$

$1.21000000 E+02$

$1.08700000 E+02$

$1.21600000 E+02$

$1.07400000 \mathrm{E}+02$

1. $10600000 E+02$

$1.13800000 E+02$

$1.06300000 E+02$

$1.06600000 E+02$

$1.16100000 E+02$

$1.14500000 E+02$

$1.25100000 E+02$

$1.11200000 E+02$

$1.13300000 E+02$

1.14500000E+02

$1.14400000 E+02$

1.10600000E+02

$1.16700000 E+02$

$1.11600000 E+02$

$1.13100000 E+02$

$1.12000000 E+02$

$1.14600000 E+02$

$1.12600000 \mathrm{E}+02$

$1.15000000 E+02$

$1.08800000 E+02$

$1.13100000 E+02$

9.22000000E+01

$9.47000000 E+01$

$9.70000000 E+01$

$9.87000000 E+01$

$9.52200000 \mathrm{E}+01$

$9.33000000 \mathrm{E}+01$

$3.86500000 \mathrm{E}+01$

$3.79000000 \mathrm{E}+01$
8.53652267E+01

$1.07887121 E+02$

$1.07887121 E+02$

$1.08698260 E+02$

$1.08698260 \mathrm{E}+02$

$1.08698260 E+02$

$.08698260 \mathrm{E}+02$

$1.14521486 E+02$

$1.08698260 E+02$

$1.08698260 \mathrm{E}+02$

$1.14521486 E+02$

$1.14521486 E+02$

$1.14521486 E+02$

$1.14521486 \mathrm{E}+02$

$1.14521486 \mathrm{E}+02$

$1.13636694 E+02$

$1.13636694 \mathrm{E}+02$

1.13636694E+02

$1.13636694 \mathrm{E}+02$

$1.13636694 \mathrm{E}+02$

1. 13636694E+02

$1.11328446 E+02$

$1.11328446 E+02$

$1.11328446 E+02$

$1.11328446 E+02$

$1.11328446 E+02$

$1.11328446 E+02$

$9.97898095 E+01$

$9.978980955 \mathrm{E}+01$

$9.97898095 E+01$

$9.978980955+0$

$9.97898095 E+01$

$9.97898095 \mathrm{E}+01$

5.31374759E+01

5.31374759E+01
1.27047733E+01

$6.61287924 E+00$

$1.31128792 E+01$

1.74040675E-03

1.29017404E+01

$-1.29825959 E+00$

$1.90174041 E+00$

$-7.21486181 E-01$

$-2.39825959 E+00$

$-2.09825959 E+00$

$1.57851382 E+00$

$-2.14861807 E-02$

$1.05785138 E+01$

$-3.32148618 E+00$

$-1.22148618 E+00$

8.63305871E-01

7.63305871E-01

$-3.03669413 E+00$

$3.06330587 E+00$

$-2.03669413 E+00$

$-5.36694129 E-01$

6.71554345E-01

$3.27155434 E+00$

$1.27155434 E+00$

3.67155434E+00

$-2.52844566 E+00$

1. $77155434 E+00$

$-7.58980947 E+00$

$-5.08980947 E+00$

$-2.78980947 E+00$

$-1.08980947 E+00$

$-4.56980947 E+00$

$-6.48980947 E+00$

$-1.44874759 E+01$

$-1.52374759 E+01$ 
Table 3 - BURN RATE OF FeK (E-P) AS A FUNCTION OF COMPOSITION, SURFACE FUNCTION, DENSITY, AND TEMPERATURE (continued)

$\begin{array}{ll}196 & 8.60000000 E+01 \\ 197 & 8.60000000 E+01 \\ 198 & 8.60000000 E+01 \\ 199 & 8.60000000 E+01 \\ 200 & 8.60000000 E+01 \\ 201 & 8.60000000 E+01 \\ 202 & 8.60000000 E+01 \\ 203 & 8.60000000 E+01 \\ 204 & 8.60000000 E+01 \\ 205 & 8.60000000 E+01 \\ 206 & 8.60000000 E+01 \\ 207 & 8.60000000 E+01 \\ 208 & 8.60000000 E+01 \\ 209 & 8.60000000 E+01 \\ 210 & 8.60000000 E+01 \\ 211 & 8.60000000 E+01 \\ 212 & 8.60000000 E+01 \\ 213 & 8.60000000 E+01 \\ 214 & 8.60000000 E+01 \\ 215 & 8.60000000 E+01 \\ 216 & 8.60000000 E+01 \\ 217 & 8.60000000 E+01 \\ 218 & 8.60000000 E+01 \\ 219 & 8.60000000 E+01 \\ 220 & 8.60000000 E+01 \\ 221 & 8.60000000 E+01 \\ 222 & 8.60000000 E+01 \\ 223 & 8.60000000 E+01 \\ 224 & 8.60000000 E+01 \\ 225 & 8.60000000 E+01 \\ 226 & 8.60000000 E+01 \\ 227 & 8.60000000 E+01 \\ 228 & 8.60000000 E+01 \\ 229 & 8.60000000 E+01 \\ 230 & 8.60000000 E+01\end{array}$

$1.49000000 E+01$ $1.49000000 E+01$ $1.49000000 E+01$ $1.49000000 E+01$ $1.49000000 E+01$ $1.49000000 \mathrm{E}+01$ $1.49000000 E+01$ $1.49000000 E+01$ $1.49000000 E+01$ $1.49000000 \mathrm{E}+01$ $1.49000000 E+01$ $1.49000000 E+01$ $1.49000000 \mathrm{E}+01$ $1.49000000 \mathrm{E}+01$ $1.49000000 E+01$ $1.49000000 \mathrm{E}+01$ $1.49000000 \varepsilon+01$ $1.49000000 \mathrm{E}+01$ $1.49000000 \mathrm{E}+01$ $1.49000000 \mathrm{E}+01$ $1.49000000 E+01$ $1.49000000 E+01$ $1.49000000 E+01$ $1.49000000 E+01$ $1.49000000 E+01$ $1.49000000 \mathrm{E}+01$ $1.49000000 E+01$ $1.49000000 \mathrm{E}+01$ $1.49000000 \mathrm{E}+01$ $1.49000000 E+01$ $1.49000000 E+01$ $1.49000000 E+01$ $1.49000000 \mathrm{E}+01$ $1.49000000 E+01$ $1.49000000 \mathrm{E}+01$
$5.00000000 E+00$ 5.00000000E+00

2.50000000E+DO

$2.50000000 \mathrm{E}+00$

2.50000000E+00

$3.00000000 E+00$

$3.00000000 E+00$

3.00000000E+00

3.30000000E +00

$3.30000000 E+00$

$3.30000000 E+00$

$3.50000000 E+00$

3.50000000E +00

$3.50000000 E+00$

4.00000000E +00

4.00000000E+00

4.00000000E+00

5.00000000E+00

5.00000000E+00

$2.50000000 E+00$

2.50000000E +00

2.50000000E +00

3.00000000E+00

$3.00000000 E+00$

$3.00000000 E+00$

$3.30000000 E+00$

$3.30000000 E+00$

$3.30000000 E+00^{\circ}$

3.50000000E+00

$3.50000000 E+00$

$3.50000000 E+00$

4.0000000OE+00

4.00000000E+00

4.00000000E+00

5.00000000E+00
$7.40000000 E+01$ $7.40000000 E+01$ $-5.40000000 E+01$ $-5.40000000 E+01$ $-5.40000000 E+01$ $-5.40000000 E+01$ $-5.40000000 E+01$ $-5.40000000 E+01$ $-5.40000000 E+01$ $-5.40000000 E+01$ $-5.40000000 E+01$ $-5.40000000 E+01$ $-5.40000000 E+01$ $-5.40000000 E+01$ $-5.40000000 E+01$ $-5.40000000 E+01$ $-5.40000000 E+01$ $-5.40000000 E+01$ $-5.40000000 E+01$ 2.50000000E+01 2.50000000E+01 2.50000000E+01 2.50000000E+01 2.50000000E+01 2.50000000E+01 2.50000000E+01 2.50000000E+01 2.50000000E+01 2.50000000E+01 2.50000000E+01 2.50000000E+01 2.50000000E+01 2.50000000E+01 2.50000000E+01 3.00000000E+01
4.08000000E+01 $3.91600000 E+01$ $8.37000000 E+01$ 8.97000000E+01 $8.33400000 E+01$ 9.30600000E+01 $9.20000000 E+01$ $9.01500000 E+01$ 9.05400000E+01 $8.81800000 E+01$ $9.06700000 E+01$ $8.88400000 E+01$ 8.85300000E+01 8.72300000E+01 $7.27300000 E+01$ $7.43600000 \mathrm{E}+01$ $7.41700000 E+01$ $3.03900000 E+01$ 2.94400000E+01 $1.00100000 E+02$ $9.98300000 \mathrm{E}+01$ $9.08100000 E+01$ $1.00400000 E+02$ $1.03100000 E+02$ $1.02900000 E+02$ $1.02100000 E+02$ $1.04800000 E+02$ $1.00400000 E+02$ $1.03100000 E+02$ $1.07700000 E+02$ 1.01300000E+02 8.47000000E+01 8.70000000E+01 $8.21200000 E+01$

$3.62200000 E+01$
5.313747599E+01 -1.233744759E+01 $5.31374759 E+01-1.39774759 E+01$ $8.61763655 E+01-2.47636554 E+00$ $8.61763655 E+01 \quad 3.52363446 E+00$ $8.61763655 E+01-2.83636554 E+00$ $9.19995921 E+01 \quad 1.06040787 E+00$ 9.19995921E+01 4.07873992E-04

$9.19995921 E+01 \quad-1.84959213 E+00$

$9.11148001 E+01 \quad-5.74800075 E-01$

$9.11148001 E+01 \quad-2.93480007 E+00$

$9.11148001 E+01 \quad-4.44800075 E-01$

8.88065516E+01 3.344839992E-02

$8.88065516 E+01 \quad-2.76551601 E-01$

$8.88065516 E+01-1.57655160 E+00$

$7.72679154 E+01 \quad-4.53791542 E+00$

$7.72679154 E+01 \quad-2.90791542 E+00$

$7.72679154 \mathrm{E}+01 \quad-3.09791542 \mathrm{E}+00$

$3.06155818 E+01 \quad-2.25581798 E-01$

$3.06155818 E+01-1.17558180 E+00$

$9.87415590 E+01 \quad 1.35844103 E+00$

$9.87415590 E+01 \quad 1.08844103 E+00$

$9.87415590 E+01 \quad-7.93155897 E+00$

$1.04564786 E+02 \quad-4.16478556 E+00$

$1.04564786 E+02-1.46478556 E+00$

$1.04564786 E+02-1.66478556 E+00$

$1.03679994 E+02-1.57999351 E+00$

$1.03679994 E+02 \quad 1.12000649 E+00$

$1.03679994 E+02 \quad-3.27999351 E+00$

$1.01371745 E+02 \quad 1.72825496 E+00$

$1.01371745 E+02 \quad 6.32825496 E+00$

$1.01371745 E+02 \quad-7.17450361 E-02$

$8.98331089 E+01 \quad-5.13310885 E+00$

$8.98331089 E+01 \quad-2.83310885 E+00$

$8.98331089 E+01 \quad-7.71310885 E+00$

$4.41208911 E+01 \quad-7.90089106 E+00$ 
Table 3 - BURN RATE OF FeK (E-P) AS A FUNCTION OF COMPOSITION, SURFACE FUNCTION, DENSITY, AND TEMPERATURE (continued)

$1.49000000 E+01$ $1.49000000 E+01$ 2.37000000E+01 2.37000000E+01 2.37000000E+01 2.37000000E+01 2.37000000E+01 2.37000000E+01 2.37000000E+01 2.37000000E+01 2.37000000E +01 2.37000000E +01 2.37000000E+01 2.37000000E+01 2.37000000E +01 2.37000000E+ 01 2.37000000E+01 2.37000000E+01 2.37000000E +01 2.37000000E+0 2.37000000E+0 2.37000000E +01 2.37000000E+0 2.37000000E+01 2.37000000E+01 2.37000000E+01 2.37000000E +01 2.37000000E+01 2.37000000E +01 2.37000000E+01 $2.37000000 E+01$ 2.40700000E+01 2.40700000E+01 $2.40700000 E+01$ $2.40700000 E+01$
5.00000000E+00

$5.00000000 E+00$

$3.50000000 E+00$

$3.50000000 E+00$

$3.50000000 E+00$

$3.50000000 E+00$

$3.50000000 E+00$

$3.50000000 E+00$

$3.50000000 E+00$

$3.50000000 E+00$

4. $00000000 E+00$

$4.00000000 E+00$

$4.00000000 E+00$

$4.00000000 E+00$

4.00000000E+00

4.00000000E+00

$4.00000000 E+00$

4.00000000E+00

4. $00000000 E+00$

$5.00000000 E+00$

5.00000000E+00

5.00000000E+00

5.00000000E+00

5.00000000E+00

5.00000000E+00

5. $00000000 E+00$

$5.00000000 E+00$

$5.00000000 E+00$

$5.00000000 E+00$

5.00000000E+00

5. $00000000 E+00$

$3.00000000 E+00$

$3.00000000 E+00$

3.00000000E +00

$3.00000000 E+00$
3.00000000E+01

3.00000000E+01

$7.40000000 E+01$

7.40000000E+01

$-5.40000000 E+01$

$-5.40000000 E+01$

2.50000000E+01

$2.50000000 E+01$

$1.40000000 \mathrm{E}+02$

$1.40000000 E+02$

$7.40000000 E+01$

$7.40000000 E+01$

$-5.40000000 E+01$

$-5.40000000 E+01$

$2.60000000 E+01$

$2.60000000 E+01$

2.60000000E+01

$1.42000000 E+02$

$1.42000000 E+02$

$7.40000000 E+01$

$7.40000000 E+01$

$7.40000000 E+01$

$7.40000000 E+01$

$-5.40000000 E+01$

$-5.40000000 E+01$

$-5.40000000 E+01$

-5.40000000E+01

2.50000000E+01

2.50000000E+01

2.50000000E+01

$1.50000000 E+02$

2.50000000E+01

$2.50000000 E+01$

1. 18000000E+02

1. $18000000 \mathrm{E}+02$
$3.80200000 E+01$ 4.41500000E+01 $1.20300000 E+02$

$1.24000000 E+02$

$8.94000000 E+01$

$9.38000000 E+01$

1. 12600000E +02

1.14200000E+02

$1.42100000 \mathrm{E}+02$

$1.44000000 E+02$

$1.17300000 \mathrm{E}+02$

$1.24400000 E+02$

$8.85000000 \mathrm{E}+01$

9.02000000E+01

1. $12400000 E+02$

1. 14300000E+02

$8.79000000 \mathrm{E}+01$

1.37100000E+02

$1.42400000 E+02$

$5.80200000 E+01$

$5.13300000 E+01$

7.00400000E+01

$5.05500000 E+01$

3.68000000E+01

$4.84100000 E+0$

5.10000000E+01

$3.96000000 E+01$

$5.45700000 E+01$

$6.82800000 E+01$

3.70000000E+01

8.79000000E+O1

$1.14300000 E+02$

$1.16100000 E+02$

$1.38900000 E+02$

$1.39800000 E+02$
$4.41208911 E+01$

4.41208911E+01

$1.21464579 E+02$

$1.21464579 E+02$

$9.89426845 E+01$

$9.89426845 E+01$

$1.11507878 E+02$

$1.11507878 E+02$

$1.37493299 E+02$

$1.37493299 E+02$

$1.09925942 E+02$

$1.09925942 \mathrm{E}+02$

B. $74040483 E+01$

B.74040483E+0

$1.00155885 \mathrm{E}+02$

$1.00155885 E+02$

$1.00155885 E+02$

$1.26487285 E+02$

$1.26487285 \mathrm{E}+02$

6.32736087E+01

$6.32736087 \mathrm{E}+01$

$6.32736087 E+01$

$6.32736087 \mathrm{E}+01$

4.07517147E+01

4. $07517147 E+01$

4.07517147E+01

4.07517147E+01

$5.33169081 E+01$

5.33169081E+01

5.33169081E+01

$8.19930332 E+01$

1.24626823E+02

$1.24626823 E+02$

$1.44935492 E+02$

$1.44935492 E+02$
$-6.10089106 \mathrm{E}+00$

2.91089377E-02

$-1.16457852 E+00$

$2.53542148 E+00$

$-9.54268446 E+00$

$-5.14268446 E+00$

$1.09212210 E+00$

2.69212210E+00

$4.60670129 E+00$

$6.50670129 E+00$

$7.37405767 E+00$

$1.44740577 E+01$

$1.09595172 E+00$

2.79595172E+00

$1.22441146 E+01$

$1.41441146 E+01$

$-1.22558854 E+01$

$1.06127147 E+01$

$1.59127147 \mathrm{E}+01$

$-5.25360872 E+00$

$-1.19436087 E+01$

$6.76639128 E+00$

$-1.27236087 \mathrm{E}+01$

$-3.95171466 E+00$

$7.65828534 \mathrm{E}+00$

$1.02482853 E+01$

$-1.15171466 \mathrm{E}+00$

1.25309190E+DO

$1.49630919 E+01$

$-1.63169081 E+01$

$5.90696684 E+00$

$-1.03268232 E+01$

$-8.52682324 E+00$

$-6.03549158 E+00$

$-5.13549158 E+00$ 


\section{Table 3 - BURN RATE OF FeK (E-P) AS A FUNCTION OF COMPOSITION, SURFACE FUNCTION, DENSITY, AND TEMMPERATURE (continued)}

$\begin{array}{lll}266 & 8.40000000 E+01 & 2.40700000 E+01 \\ 267 & 8.40000000 E+01 & 2.40700000 E+01 \\ 268 & 8.40000000 E+01 & 2.40700000 E+01 \\ 269 & 8.40000000 E+01 & 2.40700000 E+01 \\ 270 & 8.40000000 E+01 & 2.40700000 E+01 \\ 271 & 8.40000000 E+01 & 2.40700000 E+01 \\ 272 & 8.40000000 E+01 & 2.40700000 E+01 \\ 273 & 8.40000000 E+01 & 2.40700000 E+01 \\ 274 & 8.40000000 E+01 & 2.40700000 E+01 \\ 275 & 8.40000000 E+01 & 2.40700000 E+01 \\ 276 & 8.40000000 E+01 & 2.40700000 E+01 \\ 277 & 8.40000000 E+01 & 2.40700000 E+01 \\ 278 & 8.40000000 E+01 & 2.40700000 E+01 \\ 279 & 8.40000000 E+01 & 2.40700000 E+01 \\ 280 & 8.40000000 E+01 & 2.40700000 E+01 \\ 281 & 8.40000000 E+01 & 2.40700000 E+01 \\ 282 & 8.40000000 E+01 & 2.40700000 E+01 \\ 283 & 8.40000000 E+01 & 2.40700000 E+01 \\ 284 & 8.40000000 E+01 & 2.40700000 E+01\end{array}$

$\begin{array}{rr}3.00000000 E+00 & -5.40000000 E+01 \\ 3.00000000 E+00 & -5.40000000 E+01 \\ 3.00000000 E+00 & 7.40000000 E+01 \\ 3.00000000 E+00 & 7.40000000 E+01 \\ 3.50000000 E+00 & 2.50000000 E+01 \\ 3.50000000 E+00 & 2.50000000 E+01 \\ 3.50000000 E+00 & 1.25000000 E+02 \\ 3.50000000 E+00 & 1.25000000 E+02 \\ 3.50000000 E+00 & -5.40000000 E+01 \\ 3.50000000 E+00 & -5.40000000 E+01 \\ 3.50000000 E+00 & 7.40000000 E+01 \\ 4.00000000 E+00 & 2.50000000 E+01 \\ 4.00000000 E+00 & 2.50000000 E+01 \\ 4.00000000 E+00 & 1.37000000 E+02 \\ 4.00000000 E+00 & 1.37000000 E+02 \\ 4.00000000 E+00 & 1.20000000 E+02 \\ 4.00000000 E+00 & -5.40000000 E+01 \\ 4.00000000 E+00 & -5.40000000 E+01 \\ 4.00000000 E+00 & 7.40000000 E+01\end{array}$

$1.04900000 E+02$
$1.04900000 E+02$
$1.23200000 E+02$
$1.32900000 E+02$
$1.06500000 E+02$
$1.15500000 E+02$
$1.34660000 E+02$
$1.46240000 E+02$
$9.87100000 E+01$
$9.44900000 E+01$
$1.25400000 E+02$
$9.13600000 E+01$
$9.42000000 E+01$
$1.28100000 E+02$
$1.14300000 E+02$
$1.14700000 E+02$
$7.03200000 E+01$
$7.60000000 E+01$
$1.11200000 E+02$

$1.12061630 E+02$

$1.12061630 \mathrm{E}+02$

$1.34583524 \mathrm{E}+02$

$1.34583524 \mathrm{E}+02$

$1.21433783 E+02$

$1.21433783 E+02$

$1.43512478 \mathrm{E}+02$

$1.43512478 \mathrm{E}+02$

$1.08868589 E+02$

$1.08868589 \mathrm{E}+02$

$1.31390483 \mathrm{E}+02$

$1.09895147 E+02$

$1.09895147 \mathrm{E}+02$

$1.35086806 E+02$

$1.35086806 \mathrm{E}+02$

$1.30706088 E+02$

9.73299531E+0

$9.73299531 E+01$

$1.19851847 E+02$
$-7.16162980 E+00$ $-7.16162980 E+00$

$-1.13835239 E+01$

$-1.68352386 E+00$

$-1.49337827 E+01$

$-5.93378271 E+00$

$-8.85247787 E+00$

2.72752213E +00

$-1.01585893 E+01$

$-1.43785893 E+01$

$-5.99048333 E+00$

$-1.85351465 E+01$

$-1.56951465 E+01$

$-6.98680645 E+00$

$-2.07868065 E+01$

$-1.60060880 \mathrm{E}+01$

$-2.70099531 \mathrm{E}+01$

$-2.13299531 E+01$

$-8.65184715 E+00$

SIGMA $=$

10.6981419293 
\&్

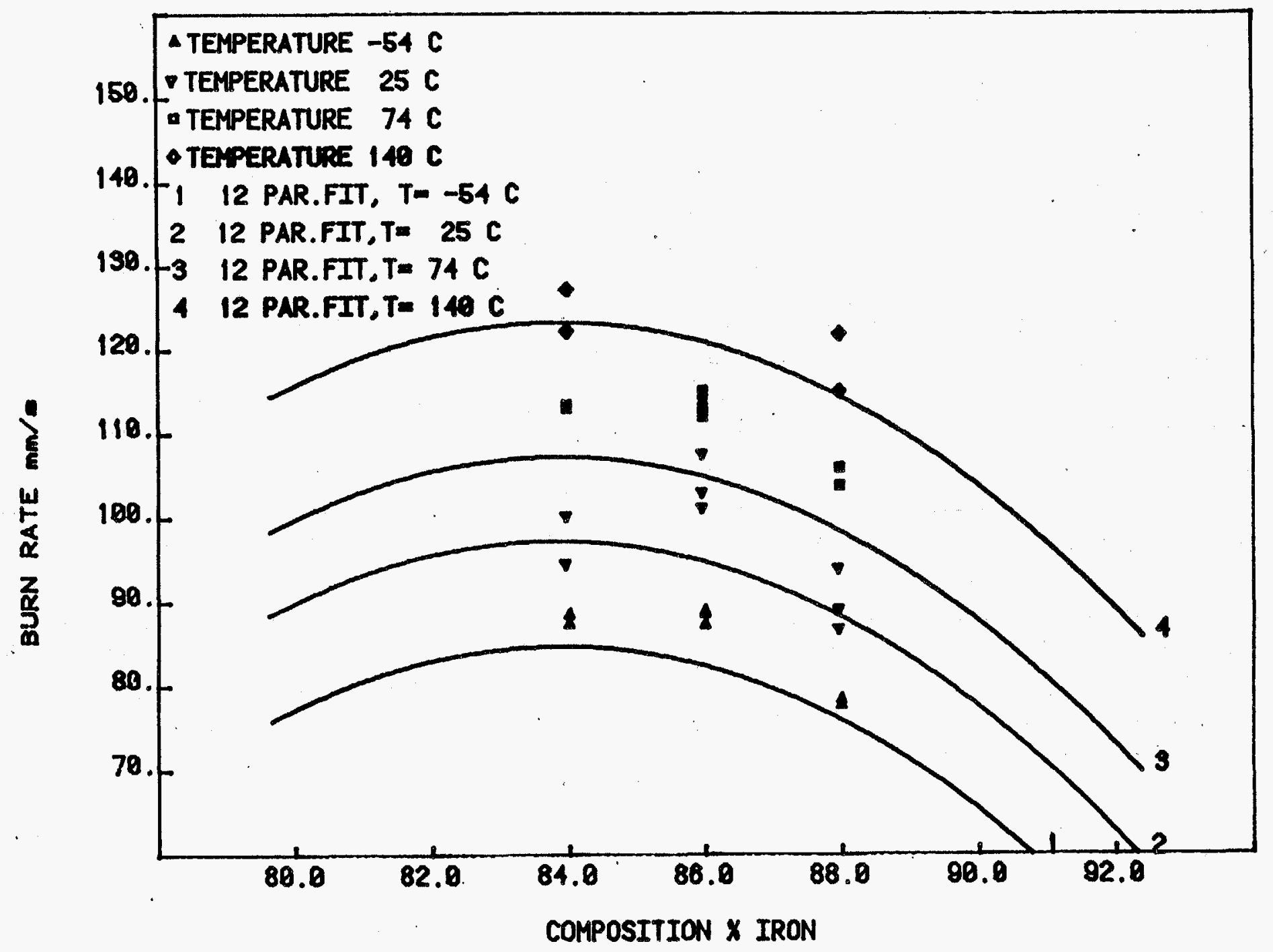

Figure 37 - Burn rate of $\mathrm{FeK}(\mathrm{E}-\mathrm{P})$ as a function of composition, surface function $=14$, density $=3.5 \mathrm{~g} / \mathrm{mL}$. 


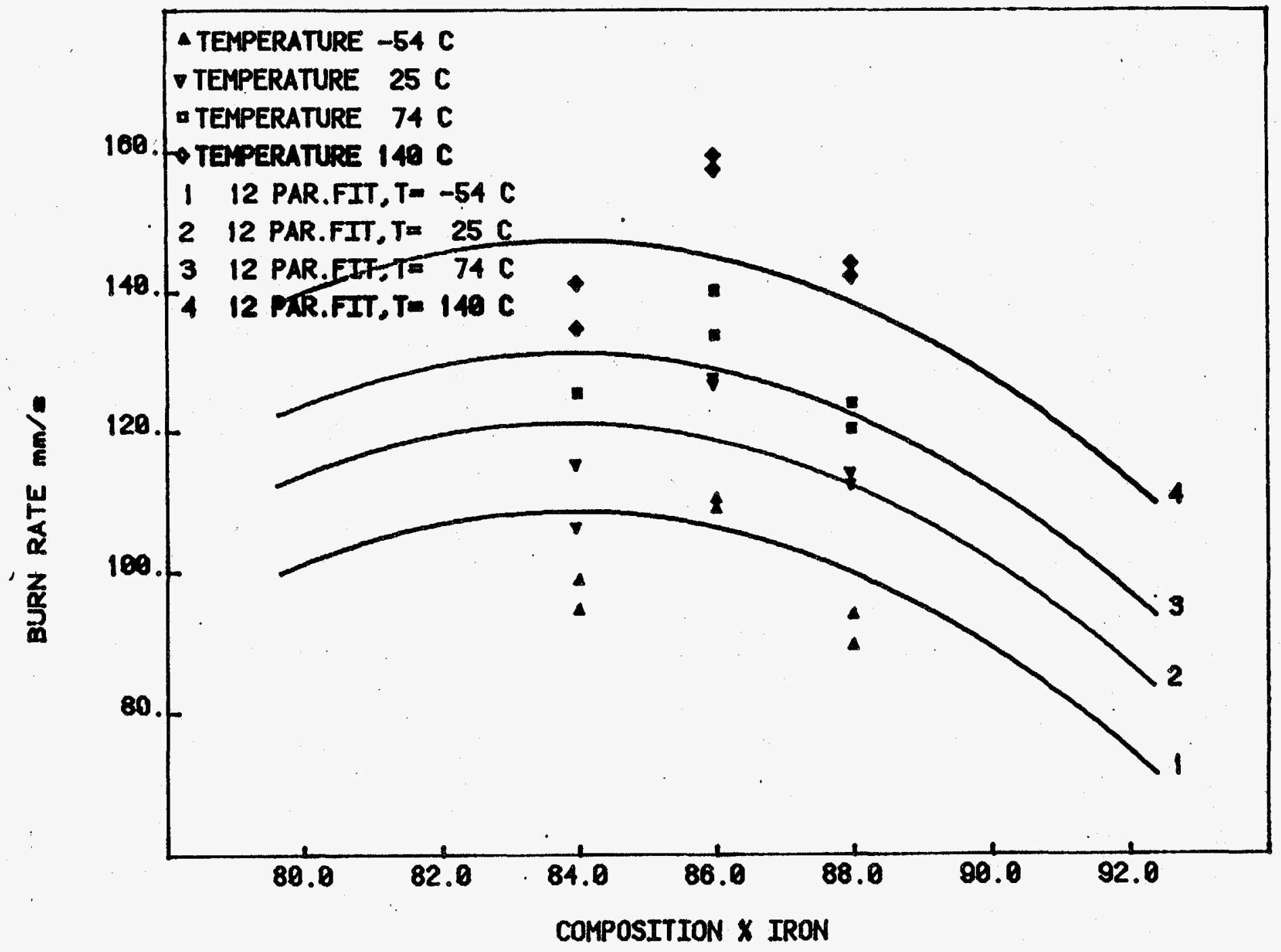

Figure 38 - Burn rate of $\mathrm{FeK}(E-P)$ as a function of composition, surface function $=24$, density $=3.5 \mathrm{~g} / \mathrm{mL}$. 
8

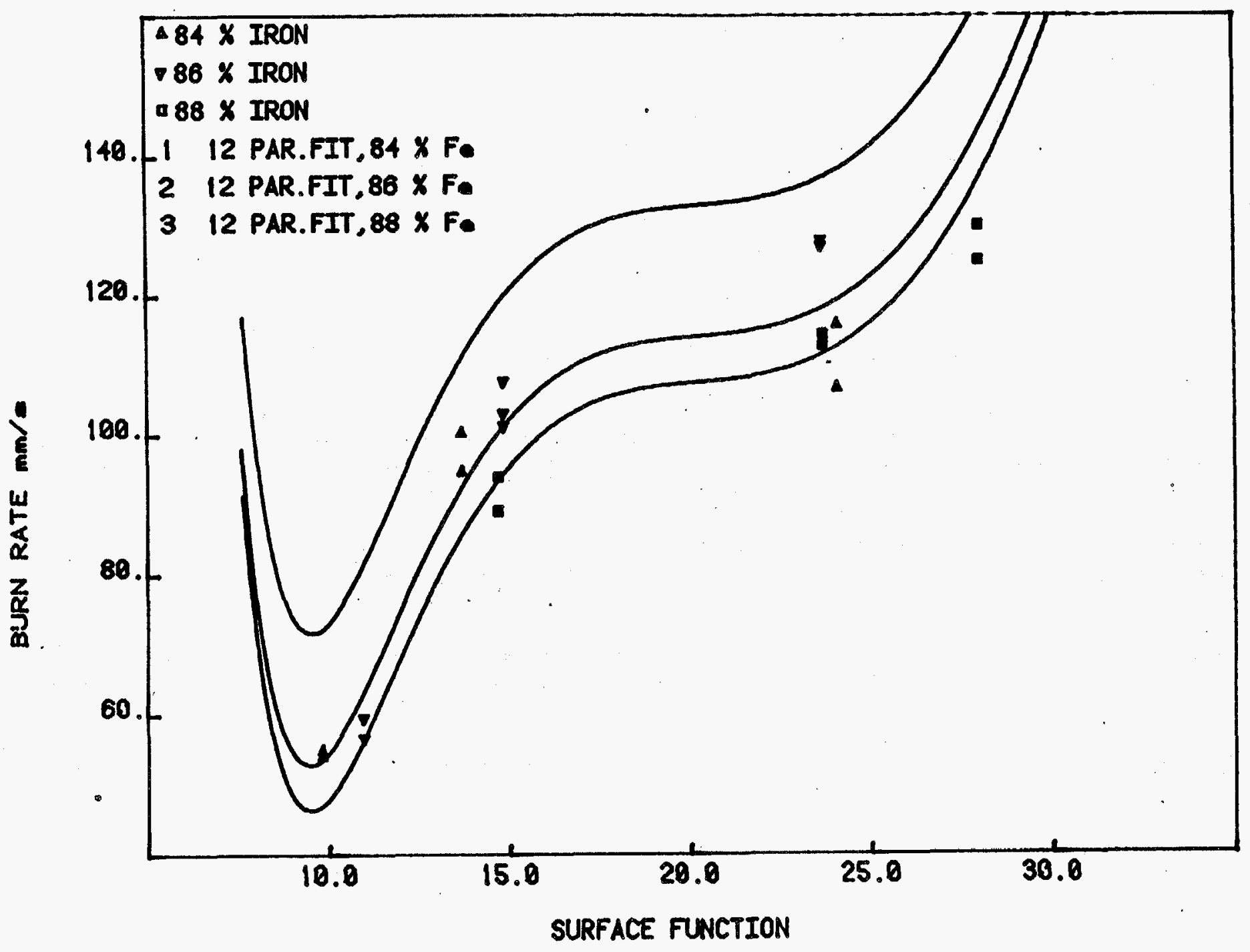

Figure 39 - Burn rate of $\mathrm{FeK}(E-P)$ versus surface function, $\mathrm{T}=25^{\circ} \mathrm{C}$, density $=3.5 \mathrm{~g} / \mathrm{mL}$. 


\section{Conclusions}

A technique for measuring burn rates of pyrotechnic heat sources producing moderate amounts of gas has been developed using a high speed video motion analysis system. The system has been shown to be fast, inexpensive to operate, easy to use and to produce reliable results. The system promises to be useful in material development, as a material product acceptance tool, and in material and device quality assurance. The limiting maximum rate is approximately 10 to 100 meters per second, depending on pellet dimensions and resolution required. Other limiting factors are the requirement that sufficient light be emitted at the burn front to allow detection, that smoke or particles are not sufficient to obscure the light, and that the compacted material does not disintegrate during measurement. The system has been used here for determining burn rates of heat source pellets of material burning at relatively slow rates.

Burn rates of the system $\mathrm{Fe} / \mathrm{KClO}_{4}(88 / 12)$ have been measured with pellets of various thickness in contact with various substrates using the high speed video burn rate analysis system to determine the effect of the substrate material on the measured burn rate values. No significant effect caused by the substrate was observed over a wide range of substrate materials. The substrate materials covered the range from thermal insulators to good thermal conductors, copper and aluminum metals, to quartz and MinK, an insulator, and substrate materials with low heat capacity to those with high heat capacities. This indicates that within reasonable conditions, the materials of construction in contact with these pyrotechnic heat pellets will have very little effect on the burn rate. The effect of pellet thickness was not measurable over the observed dimension range (about 0.1 to $1.25 \mathrm{~mm}$ thick). The effect of substrate on ease of ignition of the heat pellets was not measured, but considering the observed difficulty in ignition of some of the pellets, especially the high density pressings, any considerable heat sink may cause difficulty in ignition.

The burn rate studies (see Table 4 for a summary) described here indicate the effect of various parameters on the burn rate of pellets pressed from the pyrotechnic material $\mathrm{Fe} / \mathrm{KClO}_{4}$ obtained from two sources, UPI AND E-P. The parameters affecting the burn rate of pellets pressed from either 
Table 4 - TEMPERATURE OF PELLET REACTION ZONE

\begin{tabular}{|c|c|c|c|c|c|}
\hline SAMPLE \# & $\begin{array}{l}\text { COMPOSITION } \\
\mathrm{Fe} / \mathrm{KClO}_{4}\end{array}$ & $\begin{array}{c}\text { DENSITY } \\
\mathrm{g} / \mathrm{cc}\end{array}$ & $\begin{array}{c}\text { PELLET } \\
\text { TEMPERATURE }\end{array}$ & $\begin{array}{l}\text { BURN RATE } \\
\text { E } \mathrm{mm} / \mathrm{s}\end{array}$ & $\begin{array}{l}\text { REACTION } \\
\text { TEMPERATURE }\end{array}$ \\
\hline G5 & $88 / 12$ & 3.5 & $\begin{array}{c}-54 \\
25 \\
74 \\
140\end{array}$ & $\begin{array}{c}91.6 \\
113.4 \\
122.2 \\
143.1\end{array}$ & $\begin{array}{l}1075 \\
1137 \\
1165 \\
1234\end{array}$ \\
\hline G6 & $88 / 12$ & 4.0 & $\begin{array}{c}-54 \\
26 \\
74 \\
142\end{array}$ & $\begin{array}{l}89.4 \\
113.4 \\
120.9 \\
139.8\end{array}$ & $\begin{array}{l}1075 \\
1128 \\
1160 \\
1197\end{array}$ \\
\hline G7 & $88 / 12$ & 5.0 & $\begin{array}{c}-54 \\
25 \\
74 \\
150\end{array}$ & $\begin{array}{l}44.0 \\
53.3 \\
57.5 \\
87.9\end{array}$ & $\begin{array}{c}960 \\
1056 \\
1130\end{array}$ \\
\hline G16 & $84 / 16$ & 2.5 & $\begin{array}{c}-54 \\
23 \\
74 \\
140\end{array}$ & $\begin{array}{c}96.9 \\
100.6 \\
120.8 \\
54.6\end{array}$ & $\begin{array}{l}1203 \\
1213 \\
1221 \\
1236\end{array}$ \\
\hline G17 & $84 / 16$ & 3.0 & $\begin{array}{c}-54 \\
25 \\
74 \\
140\end{array}$ & $\begin{array}{c}96.9 \\
102.0 \\
117.8 \\
128.5\end{array}$ & $\begin{array}{l}1165 \\
1172 \\
1213 \\
1210\end{array}$ \\
\hline G18 & $84 / 16$ & 3.3 & $\begin{array}{c}-54 \\
26 \\
74 \\
140\end{array}$ & $\begin{array}{c}93.6 \\
101.3 \\
114.3 \\
130.1\end{array}$ & $\begin{array}{l}1204 \\
1193 \\
1221 \\
1220\end{array}$ \\
\hline G19 & $84 / 16$ & 3.5 & $\begin{array}{c}140 \\
-54 \\
26 \\
74 \\
140\end{array}$ & $\begin{array}{c}10.9 \\
87.9 \\
97.5 \\
112.4 \\
124.7\end{array}$ & $\begin{array}{l}1120 \\
1178 \\
1115 \\
1190 \\
1220\end{array}$ \\
\hline G20 & $84 / 16$ & 4.0 & $\begin{array}{c}-54 \\
25 \\
74 \\
140\end{array}$ & $\begin{array}{c}70.4 \\
76.4 \\
76.5 \\
95.1 \\
109.9\end{array}$ & $\begin{array}{l}1220 \\
1172 \\
1168 \\
1203 \\
1263\end{array}$ \\
\hline G21 & $88 / 12$ & 3.0 & $\begin{array}{c}-54 \\
27 \\
74 \\
140 \\
-54 \\
74\end{array}$ & $\begin{array}{l}38.5 \\
43.4 \\
58.5 \\
67.4 \\
74.2\end{array}$ & $\begin{array}{l}1105 \\
1120 \\
1172 \\
1237 \\
1051 \\
1140\end{array}$ \\
\hline G23 & $88 / 12$ & 3.5 & $\begin{array}{r}-54 \\
25\end{array}$ & $\begin{array}{l}78.0 \\
901\end{array}$ & 1095 \\
\hline & & & $\begin{array}{c}74 \\
125\end{array}$ & $\begin{array}{l}104.8 \\
118.4\end{array}$ & $\begin{array}{l}1185 \\
1213\end{array}$ \\
\hline
\end{tabular}


Table 4 - TEMMPERATURE OF PEILET REACTION ZONE (continued)

\begin{tabular}{|c|c|c|c|c|c|}
\hline G33 & $\begin{array}{l}86 / 14 \\
84 / 16 \\
84 / 16 \\
88 / 12 \\
88 / 12\end{array}$ & $\begin{array}{l}3.5 \\
4.0 \\
3.0 \\
3.5\end{array}$ & $\begin{array}{c}-54 \\
25 \\
74 \\
158 \\
-54 \\
25 \\
74 \\
140 \\
-54 \\
25 \\
74 \\
140 \\
-54 \\
27 \\
74 \\
140 \\
-54 \\
28 \\
74 \\
140 \\
-54 \\
25 \\
74 \\
140 \\
-54 \\
25 \\
74 \\
140 \\
-54 \\
27 \\
74 \\
150 \\
-54 \\
26 \\
74 \\
140 \\
-54 \\
24 \\
74 \\
135 \\
-54 \\
25 \\
74 \\
118\end{array}$ & $\begin{array}{c}72.2 \\
85.8 \\
96.4 \\
120.1 \\
50.6 \\
58.2 \\
63.2 \\
73.5 \\
43.6 \\
49.0 \\
58.0 \\
66.5 \\
49.9 \\
54.4 \\
68.4 \\
78.7 \\
42.3 \\
47.1 \\
54.5 \\
64.6 \\
107.6 \\
127.1 \\
143.0 \\
163.6 \\
108.2 \\
126.5 \\
145.8 \\
172.4 \\
105.3 \\
123.2 \\
131.8 \\
150.4 \\
109.6 \\
127.3 \\
136.9 \\
158.4 \\
99.2 \\
107.9 \\
127.8 \\
142.7 \\
104.9 \\
115.2 \\
128.1 \\
139.4\end{array}$ & $\begin{array}{l}1080 \\
1090 \\
1170 \\
1237 \\
1152 \\
1133 \\
1208 \\
1035 \\
1147 \\
1157 \\
1173 \\
1135 \\
1123 \\
1136 \\
1145 \\
1123 \\
1140 \\
1172 \\
1233 \\
1077 \\
1130 \\
1160 \\
1238 \\
1224 \\
1145 \\
1007 \\
1057 \\
1145 \\
1007 \\
1057 \\
1118 \\
1137 \\
1182 \\
1200 \\
1223 \\
1161 \\
1182 \\
1196 \\
1154 \\
1190\end{array}$ \\
\hline
\end{tabular}


material were pressing density, $\mathrm{Fe} / \mathrm{KClO}_{4}$ blend ratio, and ambient temperature of the pellet and substrate at the time of ignition (not ignition temperature, which was not measured). In addition, the blends supplied by E-P were made up from iron metal powders of several particle sizes. The effect of this parameter was also used in the correlations involving the E-P blends. The burn rate correlations of these materials are best kept separate because the particle size properties of the iron in the blends supplied by the two suppliers cannot be compared.

Both plots of effects of the various studied parameters on the burn rate of these materials and equations relating the measured burn rates to the various parameters have been presented in the previous section. The parameter plots and the related equations will be useful in material development applications and in material acceptance/quality control applications for predicting burn rates of pellets of $\mathrm{Fe} / \mathrm{KClO}_{4}$ pyrotechnic blends. Because of uncertainty of predicted values outside of the measured-range, these data should not be used outside the range of the measurements, particularly those burn rate values obtained from the fitting equations outside of the measured range. Within the range of the measurements the equations for either materials should produce satisfactory burn rate values within the reliability associated with the standard deviations presented with the parametric tables.

As seen in the results presented in the previous section, the range of parameters in the blends contained in the pressed pellets available for burn rate measurements was not wide enough to completely define the variation in burn rate with particle size and with composition. The trends appear somewhat ambiguous in a few cases. The smallest particle size iron powder was included in only the $\mathrm{Fe} / \mathrm{KClO}_{4}(88 / 12)$ blend, while the lowest particle size iron was included only in the $\mathrm{Fe} / \mathrm{KClO}_{4}(84 / 16)$ and $(86 / 14)$ blends. Extending the burn rate measurements to include pellets containing blends of these materials and compositions should improve the limited understanding of burn rate variations in these regions. An improved fit should also result if these data were available.

Although the ignition temperature was not studied, the results of a DSC study are shown in Figure 40 [1]. The figure indicates that ignition is likely 
to occur at around $490^{\circ} \mathrm{C}$ if the heating rate is high enough to overcome thermal conduction away from the ignition site.

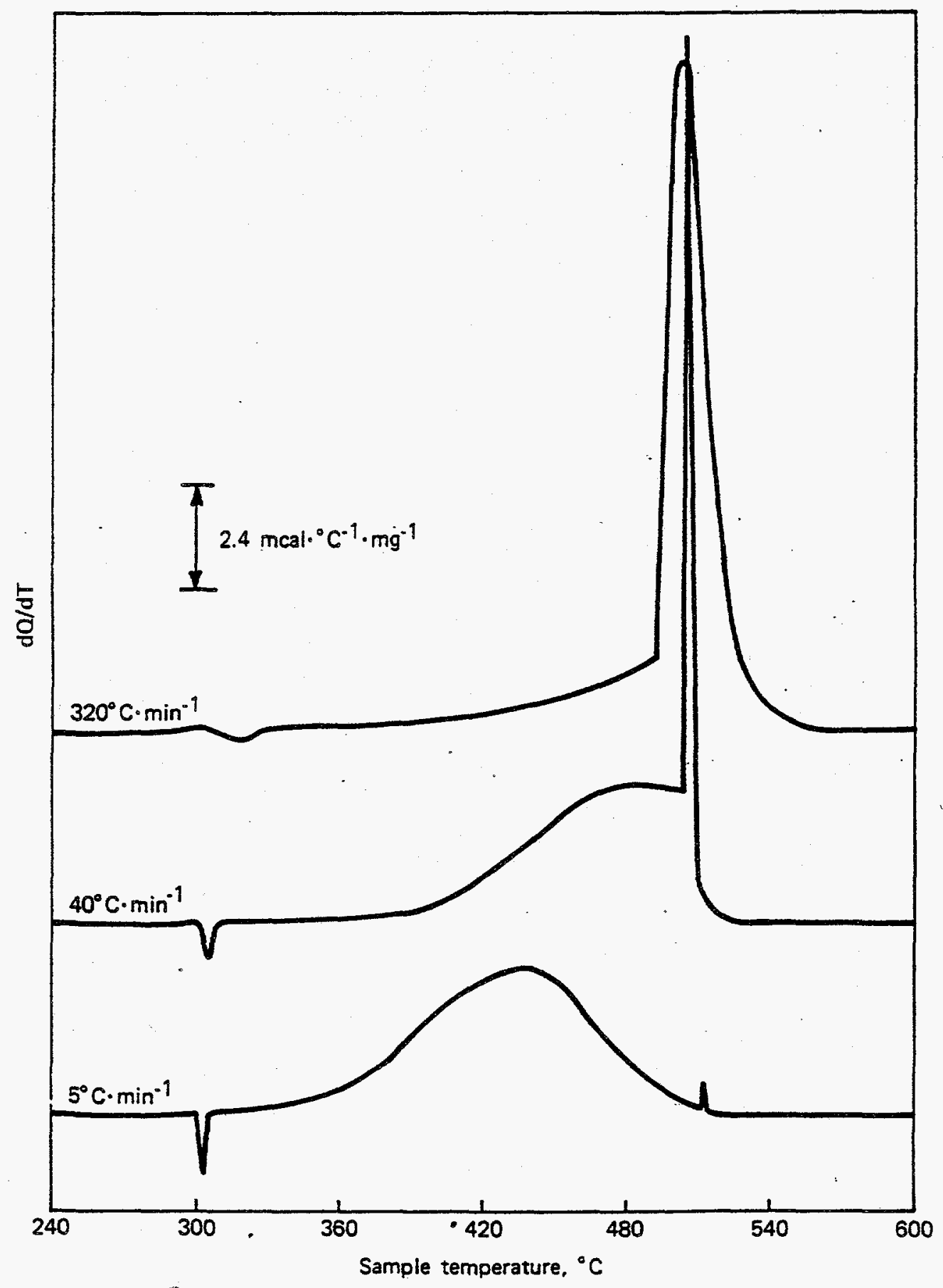

Figure 40.- Curves of $d Q / d T$ as a function of temperature for $\mathrm{Fe} / \mathrm{KClO}_{4}$ at different heating rates. 


\section{Reference}

1. C. M. Love and J. E. Glaub, DSC Investigation of the $\mathrm{Fe}+\mathrm{KClO}_{4}$ Reaction, MLM-3058, Monsanto Research Corporation (May 1983), 8pp. 


\section{Distribution}

\section{EXTERNAL}

OSTI, UC-701 (87)

D. V. Eckman, DOE/MB

R. Guidotti, Sandia National Laboratories, Albuquerque

INTERNAL

R. J. DeSando

L. R. Dosser (5)

E. N. Fray

C. Love

G. D. Miller

A. C. Munger

C. M. Woods

Technical Publications (2)

Editor, Mary J. Pacinda

Technical Publications 